\title{
IMPROVING AND EXPANDING THE CAPABILITIES OF THE POLY- PICOSATELLITE ORBITAL DEPLOYER
}

\author{
A Thesis \\ presented to \\ the Faculty of California Polytechnic State University, \\ San Luis Obispo
}

\author{
In Partial Fulfillment \\ of the Requirements for the Degree \\ Master of Science in Aerospace Engineering \\ by \\ David Pignatelli \\ October 2014
}


(C) 2014

David Pignatelli

ALL RIGHTS RESERVED

Page ii 
COMMITTEE MEMBERSHIP

TITLE:

Improving and Expanding the Capabilities of the Poly-Picosatellite Orbital Deployer

AUTHOR:

David Pignatelli

DATE SUBMITTED:

October 2014

COMMITTEE CHAIR:

Dr. Jordi Puig-Suari, Professor

Aerospace Engineering Department

COMMITTEE MEMBER:

Dr. Faysal Kolkailah, Professor

Aerospace Engineering Department

COMMITTEE MEMBER:

Dr. Eltahry Elghandour, Lecturer

Civil/Aerospace Engineering Department

COMMITTEE MEMBER:

Justin Carnahan

Tyvak Nano-Satellite Systems

Page iii 


\begin{abstract}
Improving and Expanding the Capabilities of the Poly-Picosatellite Orbital Deployer David Pignatelli
\end{abstract}

The Poly-Picosatellite Orbital Deployer (P-POD) has undergone a series of revisions over the years. The latest revision, described in this Master's Thesis, incorporates new capabilities like EMI shielding, an inert gas purge system, and an electrical interface to the CubeSats after they are integrated into the P-POD. Additionally, some mass reduction modifications are made to the P-POD, while its overall strength is increased. The P-POD inert gas purge system successfully flew, on a previous revision P-POD. The P-POD components are analyzed to a set of dynamic loads for qualification, and successfully undergoes random vibration qualification testing. The P-POD encounters some problems in thermal vacuum cycling qualification and EMI testing, but there is evidence that the issues can be mitigated. A path forward is laid out to complete both sets of testing.

Keywords: CubeSat, P-POD, EMI, Qualification

Page iv 


\section{ACKNOWLEDGMENTS}

I would like to thank my family who have always supported me and pushed me to expand my own capabilities since childhood. I would not be here today if it was not for their continued support.

I would also like to thank my soon-to-be wife, Savannah, who has always supported me and who has been patient and helpful as I put the finishing touches on this project. She deserves an award for staying by my side.

Additional thanks go out to Dr. P, Roland, and Nuge, whose guidance has proven to be extremely valuable time and time again.

And last but not least, I would like to thank all the students who have contributed to any of the work in this project, I could not have done it without you. 


\section{TABLE OF CONTENTS}

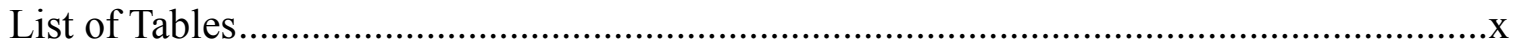

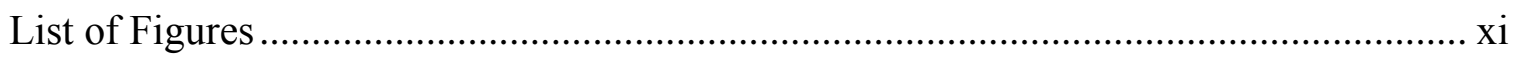

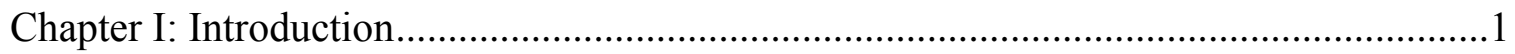

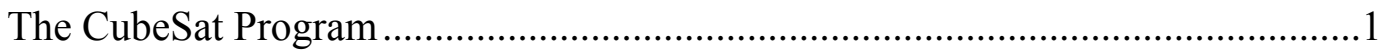

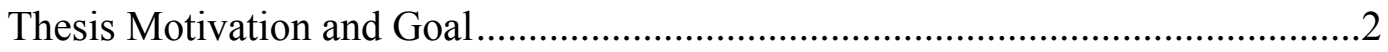

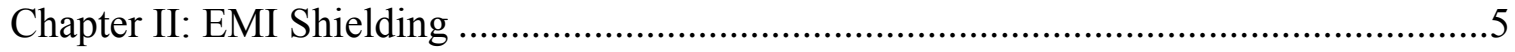

EMI/RFI Leakage Sources.........................................................................

Sealing EMI/RFI Leaks ...........................................................................

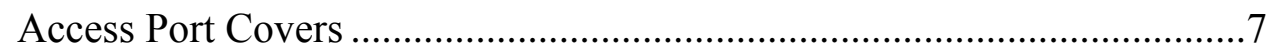

Metal to Metal Flanged Interface....................................................

Gasket Interface with Captive Screws ..........................................

Gasket Interface with Button Cap Screws .....................................10

Access Port Cover Trade Summary ..............................................12

Door/Collar Interface Gap Closure .......................................................12

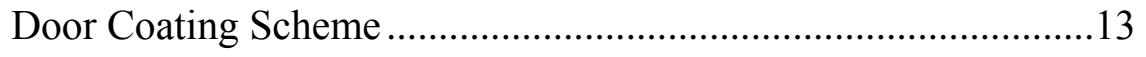

EMI Gasket Properties ............................................................................13

EMI Resistant Venting Methods ..............................................................14

Conductive Mesh Vent Hole Cover .................................................14

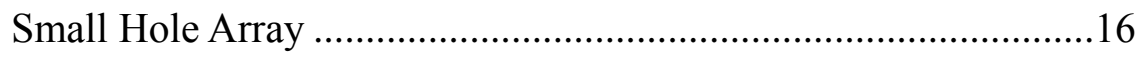

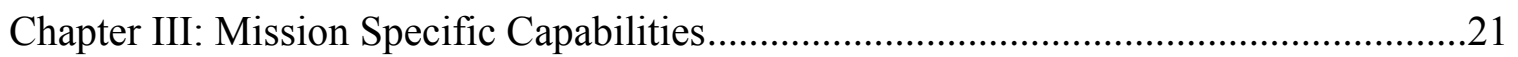

Page vi 


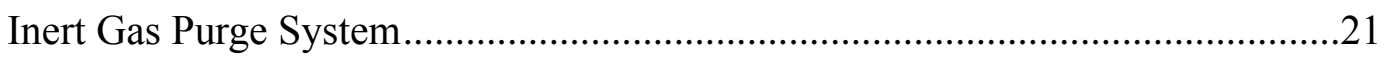

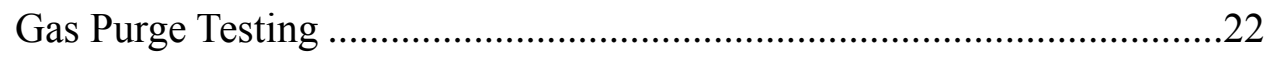

First Gas Purge Test Run .............................................................25

Second Gas Purge Test Run .........................................................26

Third and Fourth Gas Purge Test Run............................................27

Gas Purge Testing Results.................................................................29

Mk. IV Back Plate Design ......................................................................

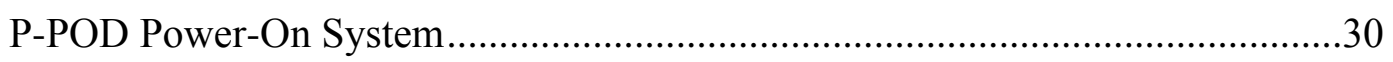

Power-On System Wireless Options ............................................................

Inductive Charging ........................................................................

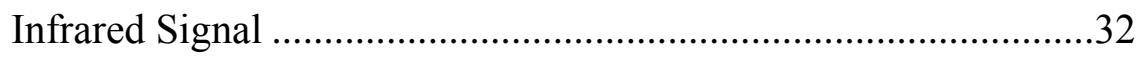

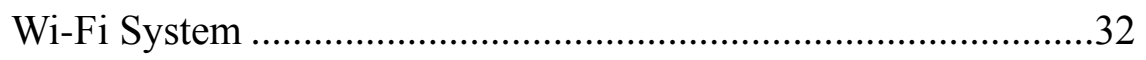

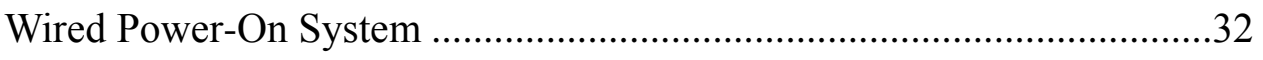

In-House Conical Spring System...................................................33

Commercial Separation Connector Solutions ...................................34

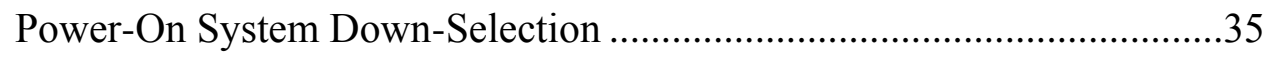

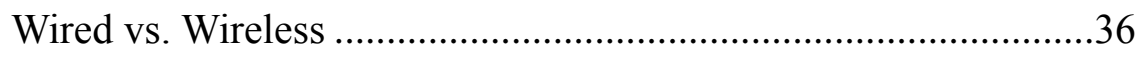

Wired System Trade Study ..............................................................

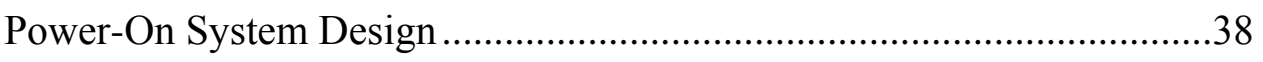

Mechanical System Design................................................................

Electrical System Design ................................................................. 40

CubeSat Interface Specification.....................................................

Power-On System Structural Analysis .....................................................42 


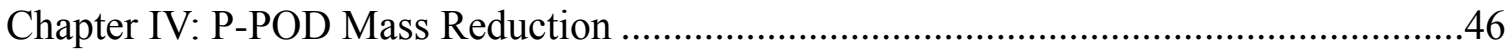

P-POD Design Loads ................................................................................46

P-POD Mk. IV Part Design and Analysis ........................................................50

P-POD Mk. IV Top Panel ............................................................50

P-POD Mk. IV Bottom Panel .........................................................53

P-POD Mk. IV Side Panel ..................................................................55

P-POD Mk. IV Back Plate .................................................................58

P-POD Mk. IV Release Mechanism Bracket.......................................61

P-POD Mk. IV Collar ....................................................................64

P-POD Mk. IV Door ...................................................................6

P-POD Mk. IV Access Port Covers ....................................................69

P-POD Mk. IV Pusher Plate ........................................................ 70

P-POD Structural Redesign Summary ...............................................73

Chapter V: Environmental Qualification and EMI testing .........................................75

Random Vibration Qualification Compliance..................................................76

Atlas V Random Vibration Qualification..........................................76

Falcon 9 Random Vibration Qualification ...........................................81

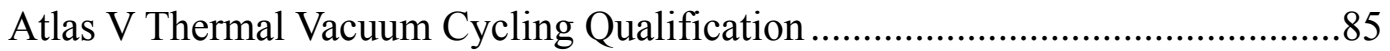

First Thermal Vacuum Cycling Anomaly ..........................................8 87

Second Thermal Vacuum Cycling Anomaly ........................................8 88

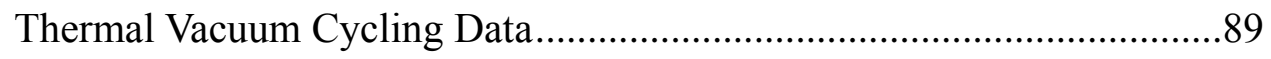


Thermal Vacuum Cycling Fault Tree Analysis

P-POD Mk. IV Thermal Vacuum Testing Path Forward ........................96

P-POD EMI Attenuation Testing ............................................................97

Dynamic Range Testing, 437 MHz..................................................98

$437 \mathrm{MHz}$ P-POD Attenuation Testing .............................................100

437 MHz P-POD Attenuation Testing Results...........................101

Dynamic Range Testing, $5.6 \mathrm{GHz}$..................................................103

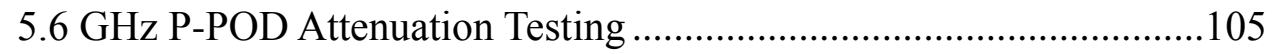

5.6 GHz P-POD Attenuation Testing Results ..........................105

P-POD Attenuation Testing Summary and Path Forward.....................106

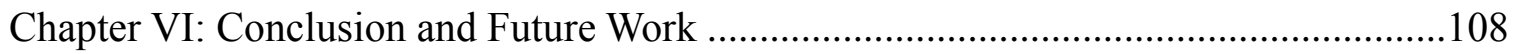

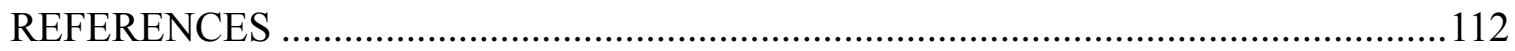




\section{LIST OF TABLES}

Table 1 Access Port Cover Trade Study Summary (1-3, 3 is best)............ 12

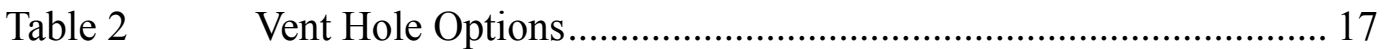

Table 3 Common CubeSat Frequencies................................................ 18

Table $4 \quad$ 1/16" Hole Pattern Attenuation Results ....................................... 19

Table 5 3/64" Hole Pattern Attenuation Results ...................................... 19

Table $6 \quad$ Purge Test Data for Run 1 .................................................... 26

Table $7 \quad$ Purge Test Data for Run 2 …............................................... 27

Table $8 \quad$ Purge Test Data for New Taping Method.................................... 28

Table 9 P-POD Electrical Interface Trade Summary $(1-5,5$ is best)......... 37

Table $10 \quad$ Wired Interface Trade Summary ............................................. 38

Table 11 Back Plate Fundamental Frequencies ...................................... 45

Table 12 VRS Input Values and Output Loads ...................................... 49

Table 13 NPSCuL to Aft Bulkhead Carrier Levels .................................. 76

Table $14 \quad$ NPSCuL to Aft Bulkhead Carrier Qualification Levels................ 77

Table 15 Falcon 9 Short Duration Qualification Levels ............................ 82

Table $16 \quad$ Fault-Tree Analysis Investigation .......................................... 93

Table 17 P-POD Shielding Effectiveness at $437 \mathrm{MHz}$.......................... 102 


\section{LIST OF FIGURES}

Figure 1 Cubesats and their Deployment Systems .................................. 1

Figure 2 P-POD Coordinate System and Component Diagram .................... 3

Figure 3 Examples of EMI Leaking Gaps............................................. 5

Figure $4 \quad$ Sources of EMI Leakage ........................................................ 6

Figure 5 P-POD Mk. III Rev. E. Access Port ........................................ 7

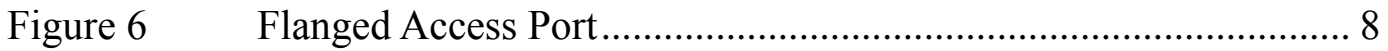

Figure $7 \quad$ Captive Screw ................................................................. 9

Figure $8 \quad$ Captive Screw Access Port Design........................................... 10

Figure $9 \quad$ Example of Torx-Drive Button Cap Screw ................................ 11

Figure $10 \quad$ Button Cap Screw Flanged Access Port Design ......................... 11

Figure $11 \quad$ Door Coating Scheme ........................................................ 13

Figure 12 Side Panel Vent Hole with Mesh. .............................................. 15

Figure $13 \quad$ Top Panel Vent Hole with Mesh. ............................................. 16

Figure 14 EMPro Attenuation Plots at $10 \mathrm{GHz}$....................................... 19

Figure 15 P-POD Mk. III Rev. E Nitrogen Purge Back Plate..................... 22

Figure $16 \quad$ Nitrogen Purge Test Setup .................................................... 23

Figure $17 \quad$ Purge Test P-POD Back Plate .............................................. 24

Figure $18 \quad$ First Side Panel Taping Method ................................................ 25

Figure 19 Reinforced Taping Pattern ................................................. 28

Figure $20 \quad$ Internal Pressure vs Inlet Flow Rate ....................................... 29

Figure 21 P-POD Nitrogen Purge Back Plate Design................................ 30

Figure 22 P-POD Power-On System Configuration. ................................. 33 
Figure 23 Wired Interface Without Connector.

Figure 24 Glen-Air Zero Ejection Force Connector ................................... 35

Figure 25 Planetary Systems Corporation Separation Connector ................ 35

Figure 26 Separation Connector Exploded View. ...................................... 39

Figure 27 P-POD Power-On System Circuit Drawing............................... 41

Figure 28 CubeSat Connector Interface ............................................... 42

Figure $29 \quad$ Power-On Access Port FEM ............................................... 43

Figure $30 \quad$ Power-On Access Port FEA Results ...................................... 43

Figure 31 1st Mode of Power-On/Purge Style Back Plate.......................... 44

Figure $32 \quad$ NPSCuL Acceptance Loads at P-POD Interface ....................... 47

Figure 33 P-POD Mk. III Rev. E Top Panel............................................ 51

Figure 34 P-POD Mk. IV Top Panel ................................................... 52

Figure 35 P-POD Mk. IV Top Panel FEA Results ................................... 53

Figure 36 P-POD Mk. III Rev. E Bottom Panel....................................... 53

Figure 37 P-POD Mk. IV Bottom Panel ................................................ 54

Figure 38 P-POD Mk. IV Bottom Panel FEA Results ............................... 55

Figure 39 P-POD Mk. III Rev. E Side Panel.......................................... 56

Figure $40 \quad$ P-POD Mk. IV Side Panel ....................................................... 57

Figure $41 \quad$ Side Panel FEA Results ....................................................... 58

Figure 42 P-POD Mk. III Rev. E Back Plate........................................... 59

Figure 43 P-POD Mk. IV Back Plate ..................................................... 59

Figure $44 \quad$ P-POD Mk. IV Back Plate Purge Interface................................. 60

Figure $45 \quad$ P-POD Mk. IV Back Plate FEA Results ................................... 61 


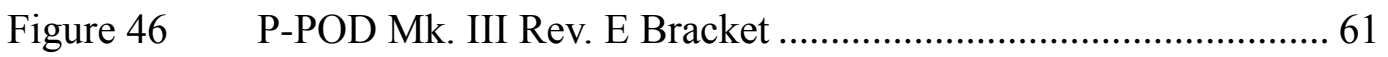

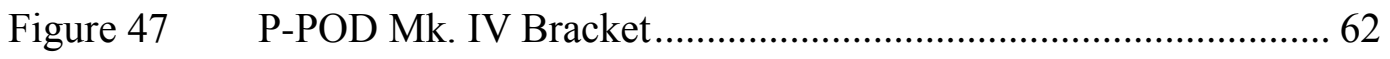

Figure 48 P-POD Mk. IV Bracket FEA Results..................................... 63

Figure 49 P-POD Mk. IV Collar with Gasket Groove ............................... 64

Figure $50 \quad$ P-POD Mk. IV Collar FEA Results ........................................ 65

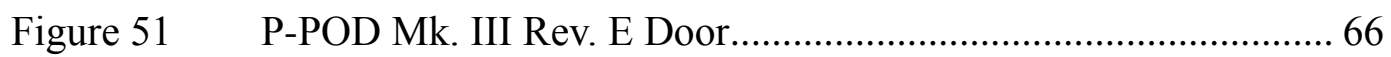

Figure 52 P-POD Mk. IV Door Design............................................... 67

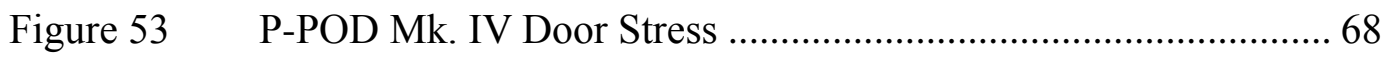

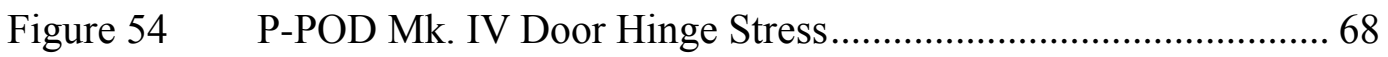

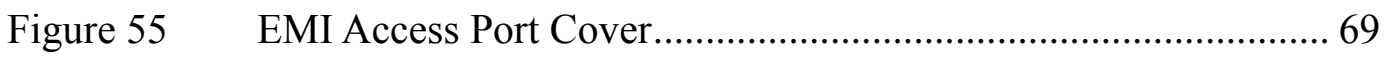

Figure 56 P-POD Mk. IV Large Access Port ....................................... 70

Figure 57 P-POD Mk. III Rev. E “Tuna Can” Pusher Plate........................ 71

Figure 58 P-POD Mk. IV “Tuna Can” Pusher Plate .................................. 72

Figure 59 P-POD Mk. IV Final Design Assembly .................................. 74

Figure $60 \quad$ NPSCul to Aft Bulkhead Carrier Levels ................................... 76

Figure $61 \quad$ NPSCuL to Aft Bulkhead Carrier Qualification Levels................ 77

Figure 62 P-POD Mounted to NPSCuL in Testing Configuration ................ 78

Figure 63 P-POD Z-Axis Vibration Test Date.......................................... 79

Figure 64 P-POD Y-Axis Vibration Test Data ...................................... 80

Figure 65 P-POD X-Axis Vibration Test Data ..................................... 80

Figure $66 \quad$ Falcon 9 Short Duration Qualification Levels ........................... 82

Figure 67 P-POD Mounting Configuration for CRS-3 Qualification ........... 83

Figure 68 CRS Short Duration X-Axis P-POD Response Data.................... 83 
Figure 69 CRS Short Duration Y-Axis P-POD Response Data.................... 84

Figure 70 CRS Short Duration Z-Axis P-POD Response Data .................... 84

Figure 71 P-POD Qualification Thermal Profile ...................................... 86

Figure 72 P-POD Door-to-Bracket Gap .............................................. 89

Figure 73 P-POD Control Thermocouple Readings During T-Vac Testing.. 90

Figure $74 \quad$ NEA Circuit Resistances During T-Vac Testing ........................ 91

Figure 75 Switch Circuit Resistance During T-Vac Testing ....................... 91

Figure 76 P-POD Mk. IV Switch Guide Design ....................................... 94

Figure 77 Modified Mk. IV Switch Guide Design.................................... 95

Figure $78 \quad 437 \mathrm{MHz}$ Sinclair Yagi-Uda Antenna ....................................... 99

Figure 79 Anechoic Chamber Dynamic Range....................................... 100

Figure 80 RF Signal Strength Measurements at 400-500 MHz ................ 101

Figure $81 \quad$ Horn Antenna Used for 5.6 GHz EMI Testing.......................... 104

Figure 82 Dynamic Range of Anechoic Chamber for 5.6GHz Range ....... 105

Figure 83 P-POD Attenuation Measurement at $5.5 \mathrm{GHz}$ to $5.7 \mathrm{GHz}$........ 106

Figure $84 \quad$ P-POD Mk. IV Engineering Unit........................................ 110 


\section{CHAPTER I: INTRODUCTION}

\section{The CubeSat Program}

In 1999, Dr. Jordi Puig-Suari and Dr. Bob Twiggs created a design standard that detailed the design requirements of a new type of pico-satellite called a CubeSat. The whole standard was based upon a $10 \mathrm{~cm} \times 10 \mathrm{~cm} \times 10 \mathrm{~cm}$ cube, with a mass of $1 \mathrm{~kg}$. This new standard of spacecraft could be built utilizing commercial off-the-shelf parts and cost very little compared to full-size spacecraft, which can have masses of over $1000 \mathrm{~kg}$. The CubeSat form factor grants access to space for organizations - including universities, developing countries, and small companies - that previously could not afford to build a full-size spacecraft.

CubeSats built to this standard would need to utilize a standardized dispenser, designed to endure the launch vehicle environment and protect both the primary payload on the rocket and the CubeSats themselves. As a result the Poly-Picosatellite Orbital Deployer, or P-POD, was born. CubeSats along with their P-POD deployment systems

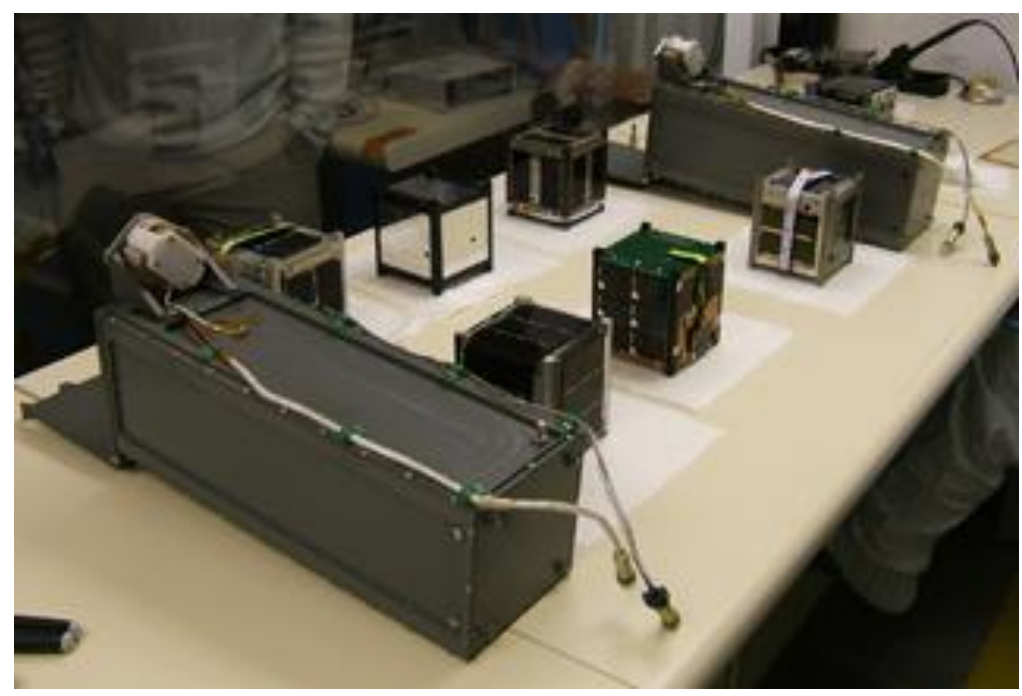

Figure 1 Cubesats and their Deployment Systems Page 1 
are shown in Figure 1. Since its inception, the P-POD has undergone a series of design improvements and increased capabilities, including a more sound structural design, CubeSat access port covers, and a more sophisticated deployment mechanism.

One concern launch providers typically have is the radiofrequency (RF) and electromagnetic (EM) interference (or RFI/EMI) that can emanate from working electronics on a satellite. This interference can potentially disrupt normal function of the launch vehicle and/or primary payload(s). In order to mitigate this risk, CubeSats are currently required to remain powered off on ascent, and cannot make any RF transmissions for a pre-determined amount of time after launch. However, if the P-POD was able to contain all RFI/EMI to an extent that would satisfy launch providers, the onascent power-off requirement could potentially be relaxed, allowing CubeSat function during launch. This could create new avenues for science and exploration, including launch environment measurement, which would bring in high fidelity data pertaining to the launch environment. A greater knowledge of different launch environments could potentially reduce recurring engineering costs and prevent over-testing. RFI/EMI containment can also support more specific mission objectives, such as inter-CubeSat communication on launch, and systems monitoring during launch.

\section{Thesis Motivation and Goal}

As CubeSat systems become increasingly complex, the need for more specific capabilities can also arise. In some instances, CubeSats can contain complex sensing instruments that require a controlled or stable environment. Sometimes this can include an Oxygen-free environment, which would require some sort of inert gas purge. In other 
instances, it can benefit the CubeSat payloads to have an electrical interface to the launch vehicle. Such an interface could provide power and data capabilities but also cannot hinder deployment in anyway. Incorporating a standard interface for these extra missionspecific capabilities can reduce recurring engineering costs and create a P-POD that can rapidly adapt to mission-specific requirements.

Throughout this Master's Thesis, different components and P-POD axes will be referred to. The figure below shows the P-POD coordinate system and notes the names of each component. The $+/-\mathrm{X}$ panels refer to the side panels, $+\mathrm{Y}$ is the Top Panel, $-\mathrm{Y}$ refers to the Bottom Panel, $+Z$ is the Door, and $-Z$ refers to the Back Plate. It is common practice to refer to panels by their coordinate representation.

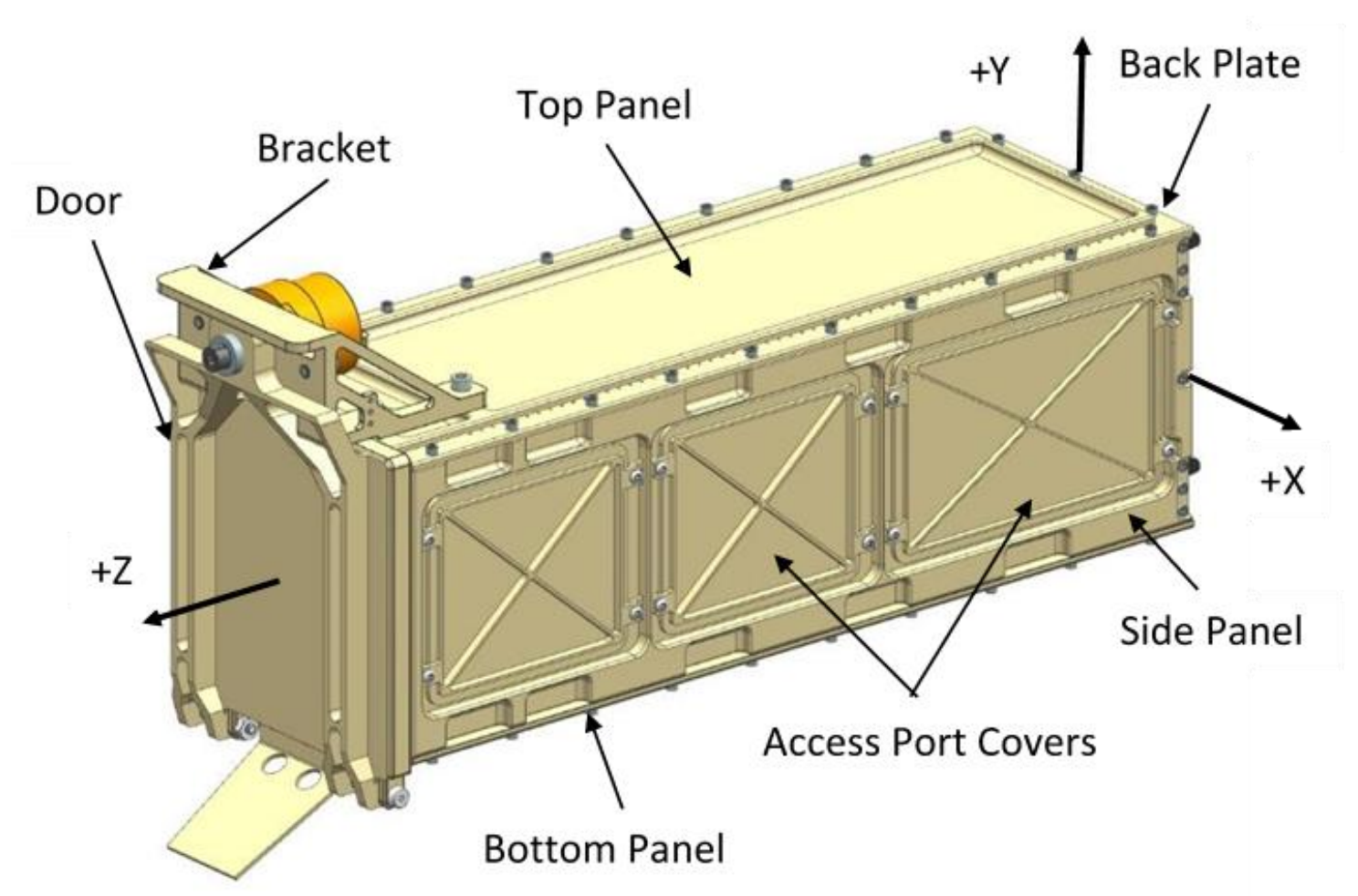

Figure 2 P-POD Coordinate System and Component Diagram Page 3 
Over the last few iterations of the P-POD, the CubeSat Program has gone to great lengths to decrease stress concentrations and increase the strength capabilities of the PPOD. While these changes have improved the P-POD, they have also increased its mass. Upon closer examination, there are some areas where material can be removed while retaining the strength added throughout the previous iterations. This will prove to be a worthwhile exercise as launch costs decrease with decreasing mass. The goal is to decrease some of the accumulated mass without negatively effecting the strength of the P-POD. In fact, another avenue explored was a review and redesign of the door, which is historically a weak point for the P-POD. In this instance, increasing strength without increasing mass is the primary objective, while also reducing mass wherever possible.

This Master's Thesis explores the possible capabilities that the P-POD can offer, while improving its base functions along the way. Supporting increasingly complex CubeSats is necessary in order to continue to further space research. CubeSats have greatly expanded the market of space exploration, allowing entities access to space, when previously it was far out of reach. Offering CubeSat Developers a more capable P-POD provides them with more tools for innovation. 


\section{CHAPTER II: EMI SHIELDING}

\section{EMI/RFI Leakage Sources}

All electronic functions inside a powered CubeSat, emit some form of electromagnetic interference. This interference can be detrimental to Launch Vehicle (LV) or primary payload function and is not tolerated during launch. For this reason, CubeSat developers are currently required to show that the CubeSat will remain powered off during launch. This not only puts limitations and requirements on the CubeSat design, but also limits the CubeSat's potential capability. If the P-POD was shown to contain all EMI/RFI, this requirement could potentially be relaxed allowing CubeSat function inside the P-POD during launch. In order to accomplish this, the sources of EMI/RFI leakage in the P-POD needed to be identified and mitigated.

EMI/RFI can escape from a container through any opening of a certain size. This size is determined based on the wavelength of the specific frequency that needs to be contained. In order to properly shield any radio frequencies or EMI, any gaps or openings must have no dimension larger than half the wavelength $(v)$ of the source. Examples of unacceptable gaps/openings are shown below in Figure 3.

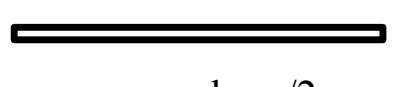

$$
1=v / 2
$$

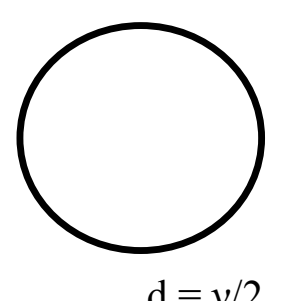

Figure 3 Examples of EMI Leaking Gaps

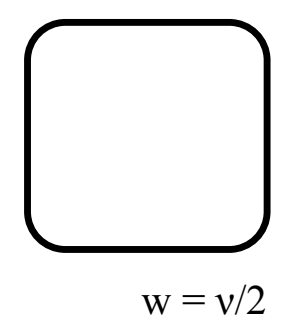


The P-POD Mk. III Rev. E has many gaps that are perfectly illustrate the reality EMI/RFI leakage. These gaps are noted below in Figure 4. Additionally, some launch vehicles require that the P-POD accommodate a venting rate that is typically accomplished through a venting hole, which becomes another area of EMI/RFI leakage. The first step in containing all EMI/RFI was to seal all gaps in the P-POD.

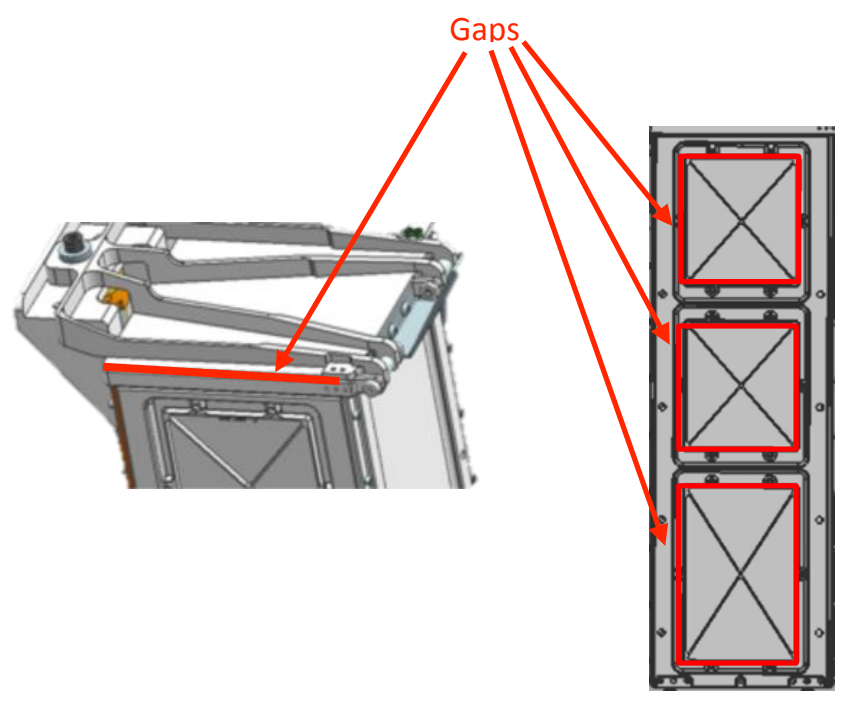

Figure 4 Sources of EMI Leakage

In order to seal these gaps, it is necessary to implement a conductive EMI gasket to any potential gaps. This includes the gap between the door and collar, as well as the gap around the access port covers. In order to gasket the access port covers, a flanged interface needed to be created to house the gasket. This mandated a redesign of the current access port design. 


\section{Sealing EMI/RFI Leaks}

P-POD components were modified to close the identified sources of EMI/RFI leakage, and incorporate EMI gaskets to any gaps. The access port covers, door/collar interface, and venting hole were all accounted for.

\section{Access Port Covers}

The Mk. III access port cover used 6 x 2-56 Socket Cap screws to affix it to the Side Panel of the P-POD. The original access port is shown below in Figure 5.

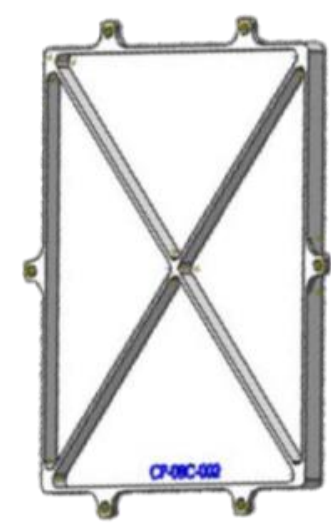

Figure 5 P-POD Mk. III Rev. E. Access Port

The 2-56 screws used in the original access port design are very small and difficult to work with. While these screws have flight heritage, it would be beneficial to incorporate larger screws into this redesign. Unfortunately, because both the P-POD exterior static envelope and interior volume must stay the same, simply increasing the screw size is impossible, as the head height of the screw cap grows significantly with increased screw sizes. Multiple options were explored in order to determine the best 
design prior to proceeding. These options include a metal to metal flanged interface, a gasket interface with captive screws, and a gasket interface with button cap screws.

\section{Metal to Metal Flanged Interface}

The first design option consisted of simply adding a flange to the existing access port cover design. This retains the 2-56 screws and all of their inherent disadvantages, but provides an interface for the EMI gasket to be compressed and contained. This design imposes minimal impact to the Side Panel design, but is unnecessarily bulky. This flanged access port is shown below in Figure 6 .

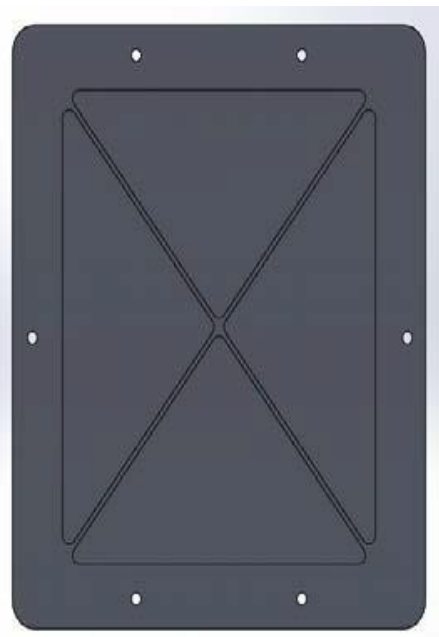

Figure 6 Flanged Access Port

\section{Gasket Interface with Captive Screws}

In an effort to move away from the 2-56 screws other design options were considered. The next design utilized captive screws. These screws consist of a small cylinder that would be press fit into the tabs on the access port cover, along with a screw that is held captive by that cylinder. These screws have a very low profile, meaning that a 
larger screw size will fit into the strict height allowance available. Captive screws are shown below in Figure 7.

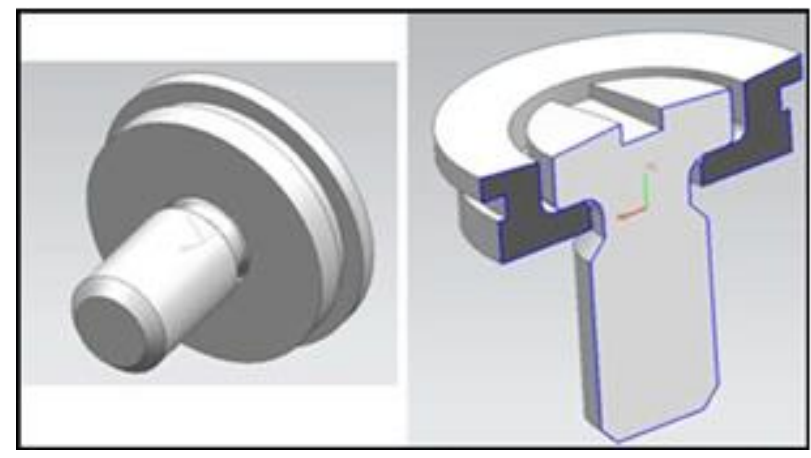

Figure 7 Captive Screw

In addition to their low profile, another benefit of these screws is that the screw itself is contained by the outer sleeve. This simplifies handling and integration operations greatly, as there is no longer a need to keep track of individual screws and also significantly less risk of foreign object debris (FOD) on the inside of the P-POD. The downside of these captive screws is that in the size necessary for this task, the only head style available is a standard flat head, which is typically not a preferred screw head to work with. This access port design is shown below in Figure 8. 

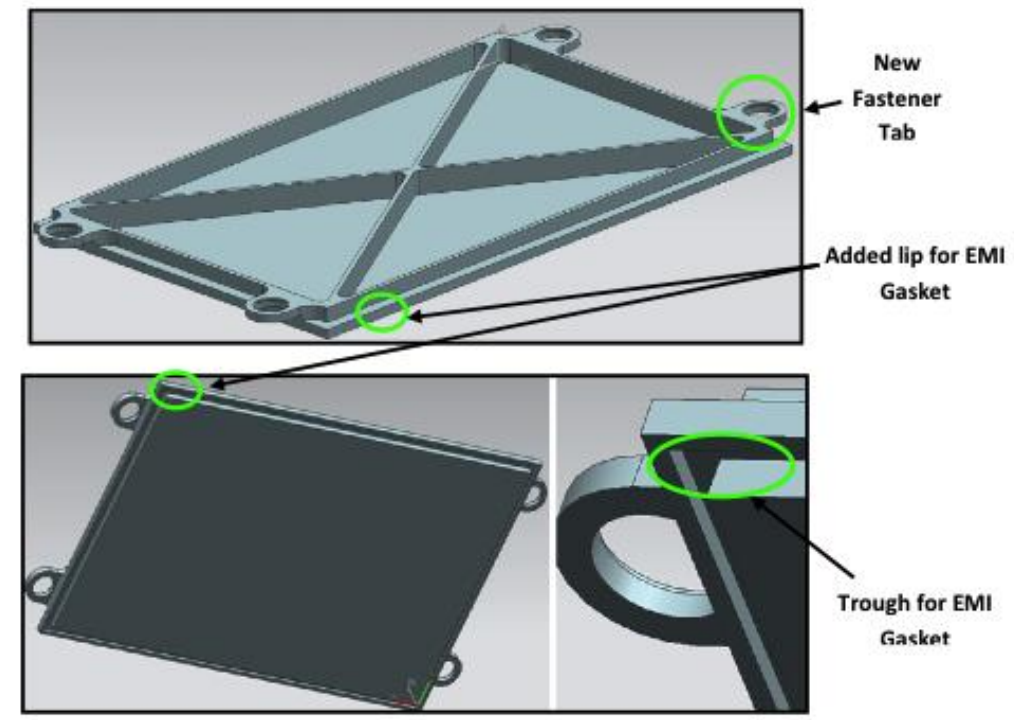

Figure 8 Captive Screw Access Port Design.

The captive screw design showed promise. However, late in the design process, the captive screw manufacturer strongly recommended using at least a whole diameter of the press-fit sleeve-in material surrounding the sleeve, to prevent the access port cover material from yielding when the sleeve is pressed into the tab. There is simply not enough space on the side panel for such large mounting tabs to be practically implemented. This necessitated the exploration of one final design option, in which the access port design itself was maintained, but the 4-40 Captive Screws were replaced by Torx-head 4-40 button cap screws.

Gasket Interface with Button Cap Screws

The Torx-drive screw used and access port cover design are shown below in Figure 9 and Figure 10. 


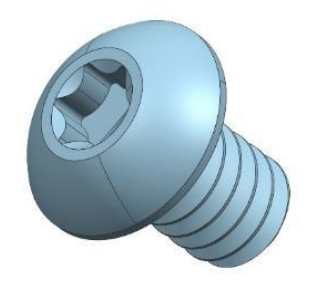

Figure 9 Example of Torx-Drive Button Cap Screw

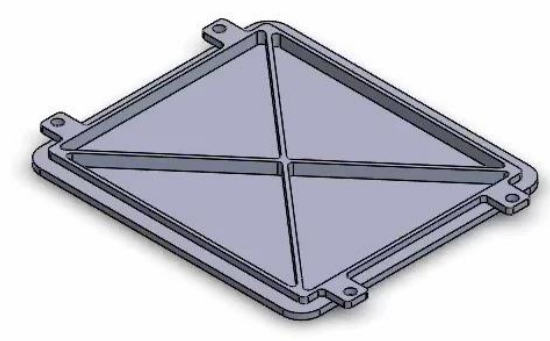

Figure 10 Button Cap Screw Flanged Access Port Design

The button cap screws have a lower profile than socket cap screws. Because of the head shape, button cap screws must utilize a smaller drive head then socket cap screws. The hex drive button cap screws are not capable of meeting the torque that is typically used on other size \#4 screws on the P-POD, which drove the requirement to use the Torx-drive button cap screws, as the star shape allows for significantly higher installation torque values. This was tested on a scrap piece of aluminum and proved able to achieve 10 in-lb of installation torque, in line with the torque applied to the rest of the 4-40 screws on the P-POD. 
Access Port Cover Trade Summary

A summary of the trade study of the various access port designs is shown below in Table 1.

Table 1 Access Port Cover Trade Study Summary (1-3, 3 is best)

\begin{tabular}{|c|c|c|c|c|c|c|c|}
\hline Design & $\begin{array}{l}\text { EMI Shielding } \\
\text { Effectiveness } \\
\text { Weight }=5\end{array}$ & $\begin{array}{c}\text { Strength/Size } \\
\text { of Fasteners } \\
\text { Weight }=4\end{array}$ & $\begin{array}{c}\text { Machinability } \\
\text { Weight }=3\end{array}$ & $\begin{array}{c}\text { P-POD } \\
\text { Design } \\
\text { Impact } \\
\text { Weight }=3\end{array}$ & $\begin{array}{l}\text { Impact to } \\
\text { Integration } \\
\text { Weight }=2\end{array}$ & $\begin{array}{l}\text { Deflection } \\
\text { Weight }=1\end{array}$ & Total \\
\hline $\begin{array}{l}\text { Current } \\
\text { Design }\end{array}$ & 1 & 1 & 3 & 3 & 2 & 1 & 36 \\
\hline $\begin{array}{l}\text { Flanged } \\
\text { Access } \\
\text { Port }\end{array}$ & 2 & 1 & 2 & 3 & 2 & 3 & 41 \\
\hline $\begin{array}{l}\text { Captive } \\
\text { Screw } \\
\text { Design }\end{array}$ & 3 & 3 & 1 & 1 & 3 & 1 & 41 \\
\hline $\begin{array}{l}\text { Button } \\
\text { Cap } \\
\text { Design }\end{array}$ & 3 & 3 & 1 & 2 & 2 & 1 & 43 \\
\hline
\end{tabular}

The outcome of this study was that the Button Cap design was the best possible option to implement an EMI gasket to the access port cover interfaces and eliminate the use of the tiny size 2 screws.

\section{Door/Collar Interface Gap Closure}

The Door-Collar interface typically has gap measuring less than 0.010 inches wide, but because of its length, it is a significant source of RF/EM leakage. Additionally, under load the door will deflect, producing a larger gap. Therefore, the gasket in this case needs to be compressible enough to allow for a very small door gap, but also be able to keep that gap closed throughout any possible deflection of the door. 


\section{Door Coating Scheme}

The interior surface of the door is typically hard anodized with a polytetrafluoroethylene (PTFE) impregnation to provide low friction surface, should any CubeSat surface slide against it. This coating is non-conductive, meaning that were the conductive gasket to sit between the collar and this interior surface of the door, it would not attenuate properly. Therefore, it is necessary to use a conductive ChemFilm coating around the edge of that surface. This coating scheme is visualized below in Figure 11.

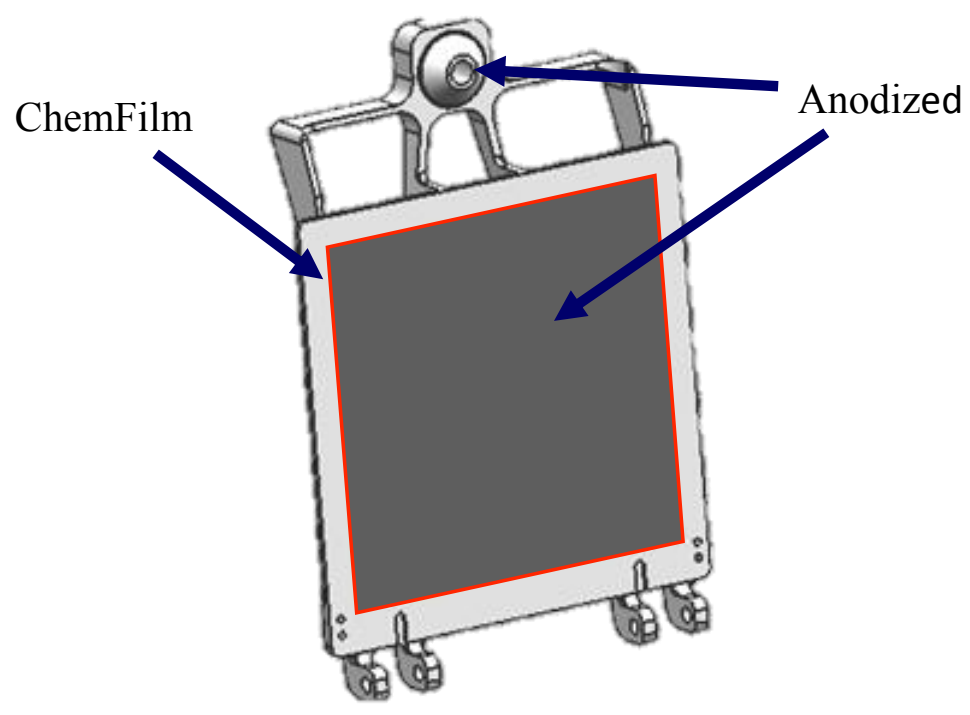

Figure 11 Door Coating Scheme

\section{EMI Gasket Properties}

The gaskets themselves, consist of a silver-plated aluminum flakes suspended in a silicone matrix. The aluminum flakes provide the conductive shield required for at least $100 \mathrm{~dB}$ of EMI attenuation, and the silicone matrix makes the gasket soft in order for it to seat and compress nicely. The gasket style chosen was a hollow cylinder extrusion, which would allow for minimal closure force. Small sized 0.053 " gaskets were chosen 
for the access ports, with an interference fit only to keep them seated. Because the access ports will not be removed on orbit, it is impossible for the gaskets to escape and create debris. The door-collar gasket could potentially escape upon CubeSat deployment, as the door opens. Therefore, a conductive epoxy must be used to keep the gasket from being dislodged when the P-POD door opens. Using a conductive epoxy also allows for a looser fitting gasket groove to provide wider range of gasket compression. This setup provides a flexible seal for EMI, which accounts for both manufacturing tolerances and deflections under load.

\section{EMI Resistant Venting Methods}

After sealing all gaps in the P-POD, the P-POD will need a ventable area. In the past, launch vehicles have required an extra hole to be machined into the P-POD to satisfy the requirement. Unfortunately, the hole-size needed to adequately vent the PPOD volume would be large enough to allow for RFI/EMI leakage, therefore, it was necessary to discover an alternative method of venting that would maintain RFI/EMI shielding effectiveness. These options include a conductive mesh installed over a hole, or an array of small holes specifically sized and spaced such that they do not allow EMI/RFI to pass through.

\section{Conductive Mesh Vent Hole Cover}

One possible method to accomplish this was to implement a conductive mesh over a large vent hole. A conductive mesh, similar to one employed in the door of a microwave, is effective in shielding RFI/EMI if properly sized for a specific frequency. 
The traditional location of a vent hole is on the ribs of the side panel, where no mounting hole is present. An example of this vent hole with mesh is shown below in Figure 12.

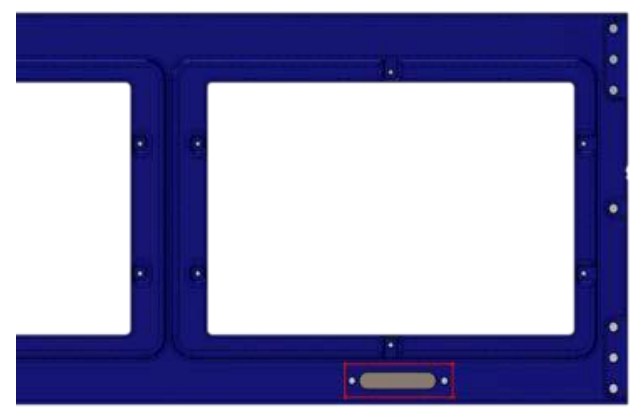

Figure 12 Side Panel Vent Hole with Mesh.

Unfortunately, the mesh interferes with the ventable area of the hole, necessitating a larger total area. Because of limitations on positioning due to fasteners through the Side Panel, this could not be accomplished by just increasing the hole size, but required the addition of multiple holes. Having multiple venting holes imposes limitations on mounting hole locations, and also requires a great deal of install time in order to affix multiple meshes to the side panels. Additionally, with so many meshes attached to the PPOD, it is difficult to keep track of all of them, making P-POD handling more difficult, and increasing the risk of damaging one of the meshes. A possible solution to mitigate some of these issues, is to put the vent hole on the thin area of the top panel, where there is ample surface area available and no impact to mounting interfaces. Having only one mesh, is much easier to keep track of, greatly reducing the risk of damage to the mesh. It also simplifies the installation, as only one mesh needs to be attached. This design is show below in Figure 13. Unfortunately, this design is not without drawbacks. With the vent 


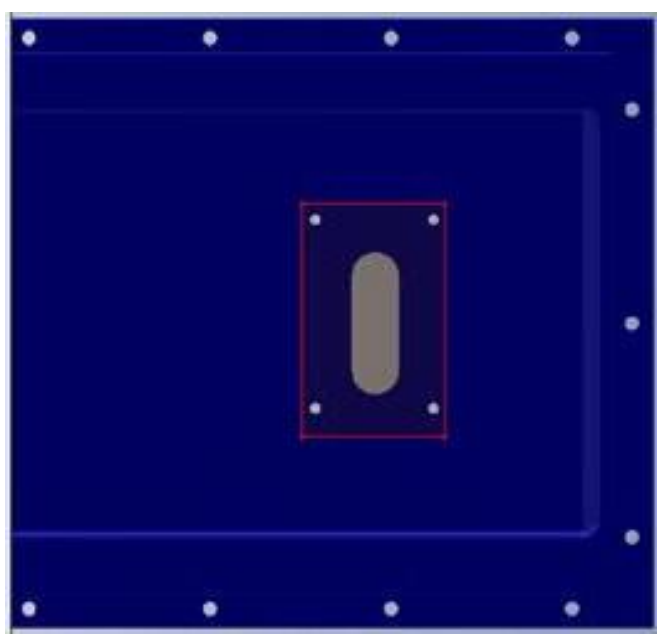

Figure 13 Top Panel Vent Hole with Mesh.

holes in the side panels, the holes lead to a vacant area of the P-POD, in the corner between the rails. Having such a large hole with direct path to the CubeSat payload, creates some risk of foreign object debris (FOD) penetrating the mesh and causing damage to the CubeSat. Additionally, the material thickness in the Top Panel is very thin, providing little thread engagement for the fasteners affixing the mesh to the Top Panel.

Small Hole Array

Another option is drilling an array of small holes in the top panel to simulate a mesh without having to attach it over a large hole in the P-POD. In order to effectively shield RFI/EMI, the holes need to be below a certain size as well as have a certain spacing. The holes need to have a hole-to-hole spacing that is not a multiple of half of the wavelength of the source, otherwise the source will not be adequately attenuated. This design, if done properly, would have the least impact on handling and installation procedures, and also has the least risk of FOD entering the P-POD due to the small size of the holes. In order to first determine what hole pattern would offer the best shielding, 
it was necessary to take a selection of patterns that should theoretically block a signal with a frequency within the specified range $(400 \mathrm{MHz}-10 \mathrm{GHz})$ from permeating through. The selection of mounting patterns are described below in Table 2 .

Table 2 Vent Hole Options

\begin{tabular}{|c|c|c|c|c|}
\hline Drill Size & Diameter & $\begin{array}{c}\text { Cutoff Frequency } \\
(\mathrm{GHz})\end{array}$ & Number of Holes & Hole Spacing (in) \\
\hline \hline "3/64" & 0.0469 & 125.92 & 112 & 0.22 \\
\hline$" 1 / 16 "$ & 0.0625 & 94.49 & 61 & 0.39 \\
\hline$" 5 / 64 "$ & 0.0781 & 75.61 & 39 & 0.62 \\
\hline$" 3 / 32 "$ & 0.0938 & 62.96 & 27 & 0.9 \\
\hline$" 7 / 64 "$ & 0.1094 & 53.98 & 20 & 1.21 \\
\hline
\end{tabular}

The plan is for these holes to be placed in two lines, on opposite sides of the Top Panel. In theory, each of these patterns should work as the cutoff frequency is much larger than $10 \mathrm{GHz}$, but a simplified model analyzed in EMPro would help to determine the best possible hole pattern. To do this, a rectangular box model with similar dimensions to the P-POD was created in CAD. Five separate models were used, each containing a hole pattern of interest on one of the long sides of the P-POD, and then imported into EMPro. A monopole antenna was inserted into the simplified model, with a parameterized antenna length to $1 / 4$ of the wavelength. The simulation was ran with five common frequencies spanning the desired range. These frequencies are shown below in Table 3. 
Table 3 Common CubeSat Frequencies

\begin{tabular}{|c|}
\hline Frequency \\
\hline $437 \mathrm{MHz}$ \\
\hline $900 \mathrm{MHz}$ \\
\hline $2.4 \mathrm{GHz}$ \\
\hline $5.6 \mathrm{GHz}$ \\
\hline $10.0 \mathrm{GHz}$ \\
\hline
\end{tabular}

As expected, the largest hole pattern with 7/64" diameter holes had trouble attenuating $10 \mathrm{GHz}$, with a minimum attenuation value of $46 \mathrm{~dB}$, below the desired $50 \mathrm{~dB}$ attenuation. Two EMI plots are shown below in Figure 14, comparing the large hole pattern with the small hole pattern at $10 \mathrm{GHz}$. The smaller hole pattern had a minimum attenuation of $76 \mathrm{~dB}$ at $10 \mathrm{GHz}$, which exceeds the desired level of attenuation. In an effort to minimize cost, the $1 / 16$ " hole pattern would be preferred, but unfortunately it was just shy of being able to attenuate the $10 \mathrm{GHz}$ signal adequately. Results for the 1/16" and 3/64" are shown below in Table 4 and Table 5, respectively. 


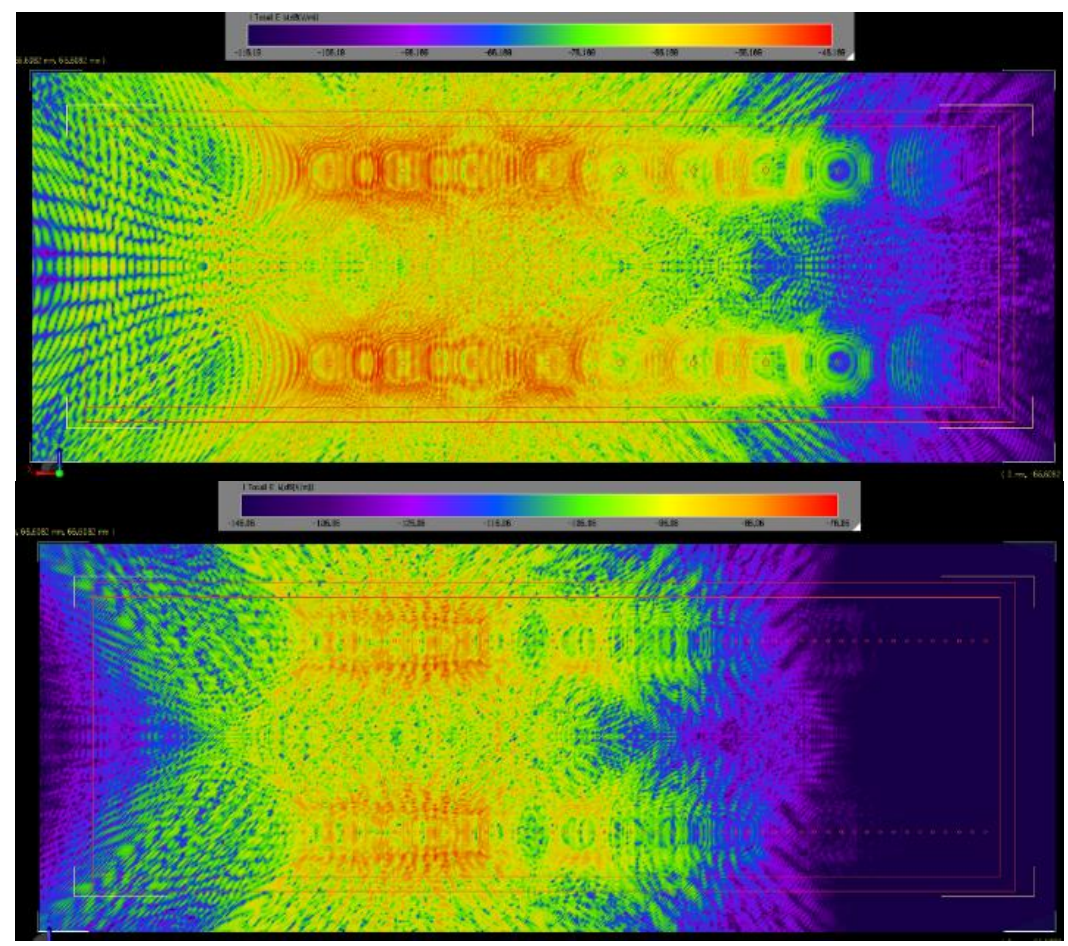

Figure 14 EMPro Attenuation Plots at $10 \mathrm{GHz}$.

Table 4 1/16" Hole Pattern Attenuation Results

\begin{tabular}{|c|c|c|}
\hline Frequency & Antenna Length $(\mathrm{mm})$ & $\begin{array}{c}\text { Worst Case Attenuation } \mathrm{dB} \\
(\mathrm{V} / \mathrm{m})\end{array}$ \\
\hline \hline $437 \mathrm{MHz}$ & 171 & 68.81 \\
\hline $900 \mathrm{MHz}$ & 83.3 & 87.30 \\
\hline $2.4 \mathrm{GHz}$ & 31.3 & 62.50 \\
\hline $5.6 \mathrm{GHz}$ & 13.4 & 54.69 \\
\hline $10 \mathrm{GHz}$ & 7.5 & 48.62 \\
\hline
\end{tabular}

Table 5 3/64" Hole Pattern Attenuation Results

\begin{tabular}{|c|c|c|}
\hline Frequency & Antenna Length $(\mathrm{mm})$ & $\begin{array}{c}\text { Worst Case Attenuation } \mathrm{dB} \\
(\mathrm{V} / \mathrm{m})\end{array}$ \\
\hline \hline $437 \mathrm{MHz}$ & 171 & 81.44 \\
\hline $900 \mathrm{MHz}$ & 83.3 & 105.05 \\
\hline $2.4 \mathrm{GHz}$ & 31.3 & 71.95 \\
\hline $5.6 \mathrm{GHz}$ & 13.4 & 83.67 \\
\hline $10 \mathrm{GHz}$ & 7.5 & 76.06 \\
\hline
\end{tabular}


Because the attenuation at $10 \mathrm{GHz}$ was under the required $50 \mathrm{~dB}$ with the $1 / 16$ " hole pattern, it was decided to proceed with manufacturing top panels with the $3 / 64$ " hole pattern. This pattern showed the highest attenuation values, which offers the most margin in case of simulation error.

Launch vehicles delegate volume to ventable area ratio requirements in order to simplify flow analysis. The Falcon 9 Launch Vehicle requires this ratio be less than 1700 inches. Using the empty P-POD volume, with pusher plate, and the 112 3/64 inch diameter holes total ventable area, this ratio is described by in $\frac{P-P O D \text { Volume }}{\text { Ventable Area }}=\frac{\mathbf{3 2 7 . i n}}{.193 \text { in }}=$ $1694 \quad$ Equation 1.

$$
\frac{P-P O D \text { Volume }}{\text { Ventable Area }}=\frac{327 . \text { in }}{.193 \text { in }}=1694
$$

This value is just under the 1700 inch requirement. CubeSat volume is ignored for simplicity, as the volume taken up by CubeSats varies significantly from case to case, which makes it difficult to predict. 


\section{CHAPTER III: MISSION SPECIFIC CAPABILITIES}

In some cases, CubeSat missions stand to benefit from specific carrier capabilities, such as inert gas purging and an electrical interface to the CubeSat while integrated into the P-POD. The inert gas purge (typically utilizing nitrogen) allows environmentally sensitive instruments to be stored for extended periods of time without degradation. Nitrogen purge consists of maintaining a positive gauge pressure in the PPOD. Because the P-POD has some small openings, a constant flow of nitrogen is used to accomplish this. In other cases, CubeSats would benefit from having a power source while integrated to the P-POD, and possible even while attached to the launch vehicle. Additionally, with that electrical interface already in place, adding additional circuits for data and systems monitoring is trivial. The most important aspect of this interface is that it does not in any way hinder CubeSat deployment. To accomplish this, different lowseparation force connectors and interfaces were considered and an interface was designed into the P-POD which could be adapted to accommodate any connector in the future that would fit in the allowable volume.

\section{Inert Gas Purge System}

The need for a nitrogen purge system for the P-POD came about when a specific CubeSat utilized a sensitive instrument as its primary payload. The instrument could not last for an appreciable duration exposed to oxygen. In order to accomplish this, the PPOD back plate was seen as the logical location for a nitrogen purge interface. The back plate was modified to accommodate an auxiliary access port to the $-\mathrm{Z}$ face of the P-POD. 
A one off Mk. III Rev. E Back Plate was manufactured for this specific case and is shown below in Figure 15.

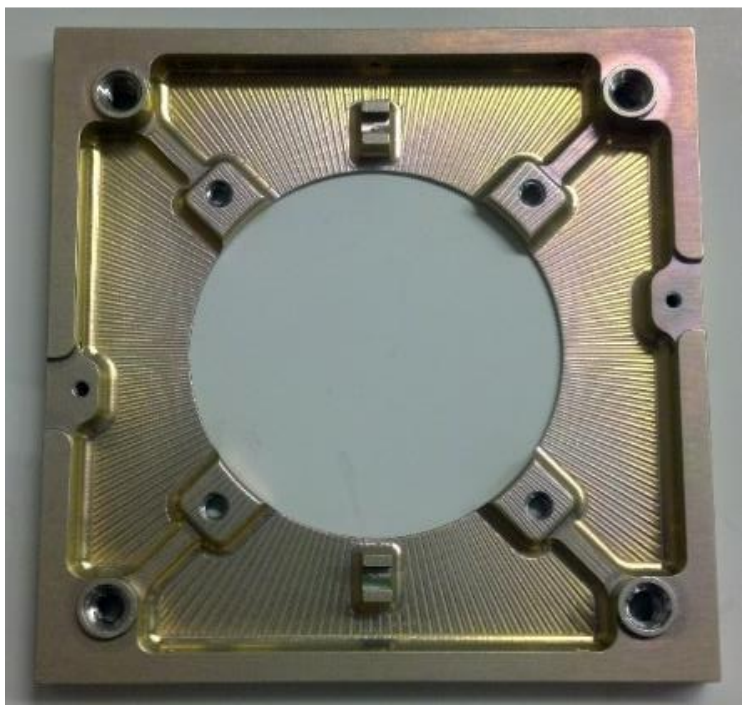

Figure 15 P.POD Mk. III Rev. E Nitrogen Purge Back Plate.

The primary design change being the addion of the central hole and cover mounting holes. Mounting holes were over designed, utilizing size 8 screws to fit the access port cover to the back plate. This strength was unnecessary, but a robust and easily workable interface was desired. Helicoils were also used to ensure there would be no damage due to repeated installation and removal.

\section{Gas Purge Testing}

In order to prove that this method would work, a test with a modified P-POD was necessary in order to show that it was capable of holding positive gauge pressure with a realistic gas flow rate. Positive gauge pressure from an influx of gas is a sign that the PPOD is being purged, meaning what was in it previously is exitting the P-POD to make 
room for the upstream gas flow. For safety reasons, air was used in place of gaseous nitrogen. Air is similar enough to nitrogen, as it is made up of primarily nitrogen, such that the safety benefit of using air instead outweighed the chance of slight errors in the pressure reading. The overall test setup is shown below in Figure 16.

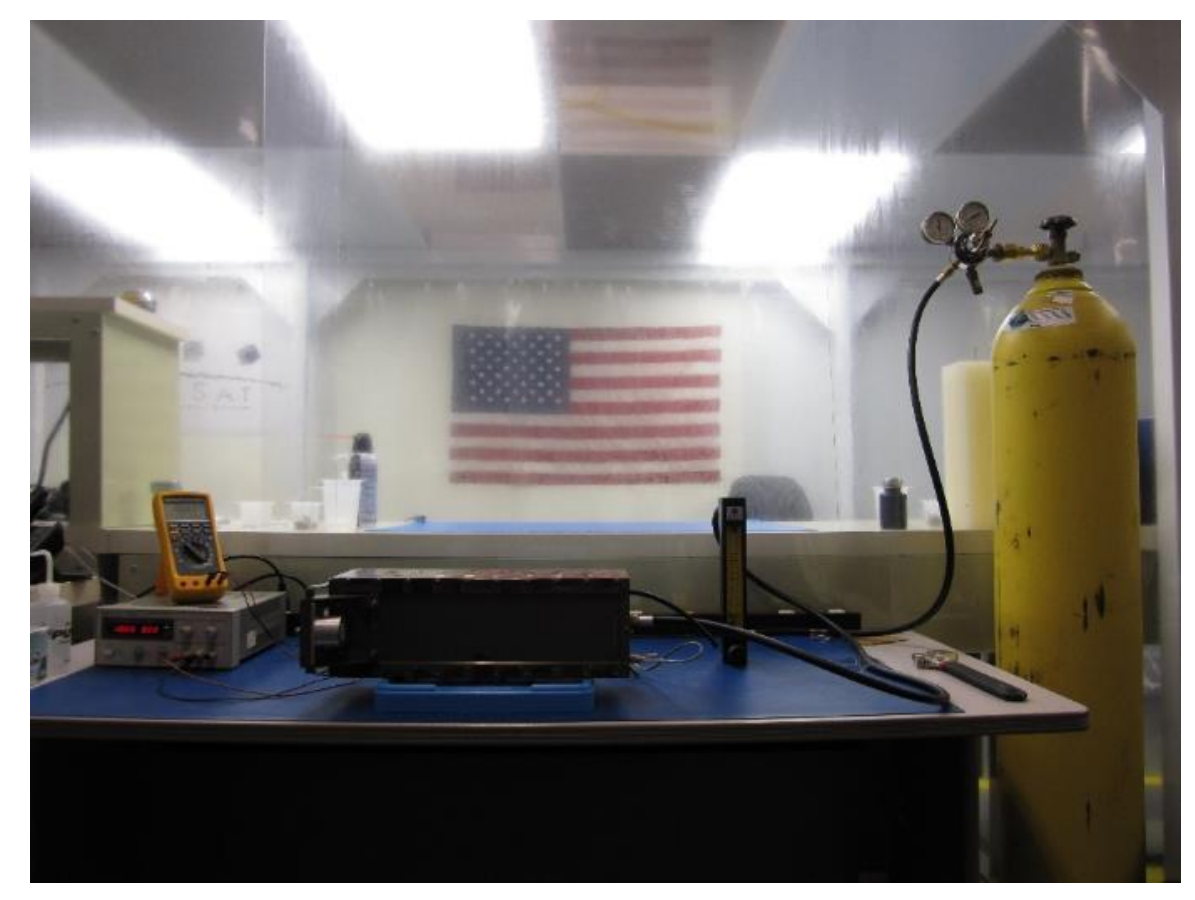

Figure 16 Nitrogen Purge Test Setup

Compressed air flows from the large tank on the right via an Accu-Trol RS-7-5 compressed gas regulator, through 0.25 " diameter hose and a Matheson FM-1127 air flow meter placed in line with flow, and then into the P-POD seen in the middle. On the left are an HP E3610A DC power supply and Fluke 189 multimeter to provide the correct power for the pressure sensor and measure the pressure sensor output respectively. Output voltage from the pressure sensor was converted to pressure units using the manufacturer calibration curve. Figure 17 shows a close up of the modified back plate 
used. The electrical wires for the pressure sensor were fed through one of the existing holes in the back plate. The black tube shown is connected to one of the orifices on the pressure sensor. Inside the black tube reads the ambient pressure, and the other orifice on the sensor reads the inner pressure. The hose at the center of the back plate is connected to a $1 / 4$ " NPT fitting and is what feeds the purge gas into the P-POD.

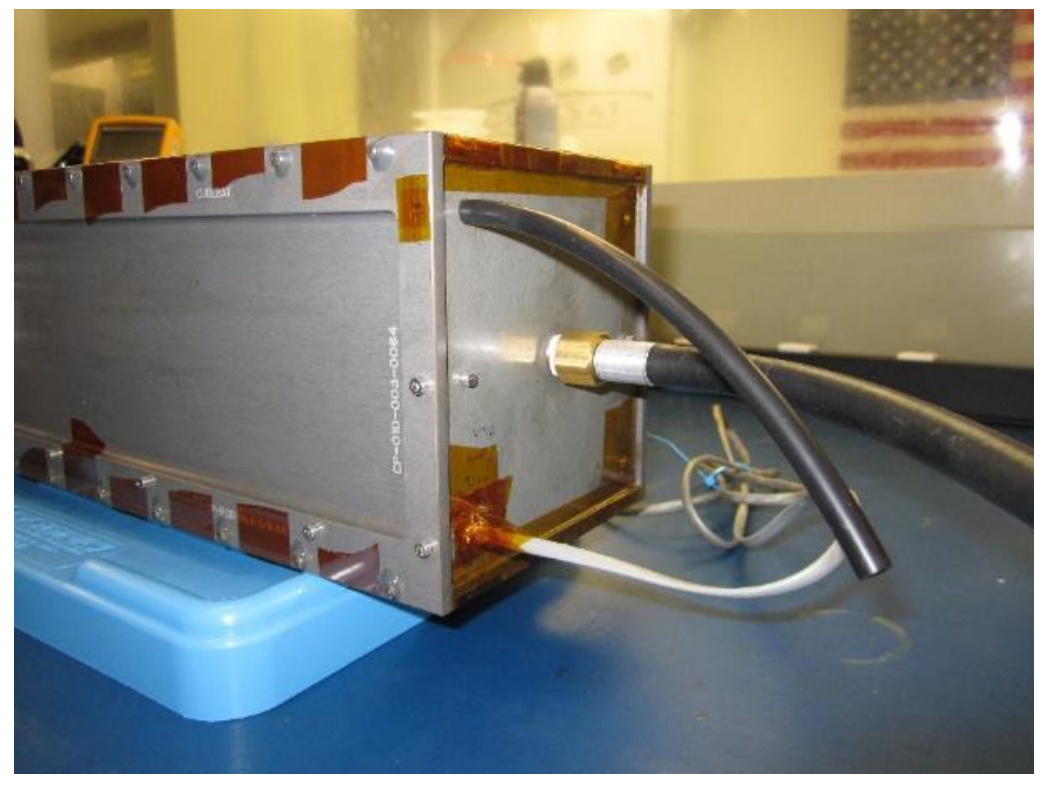

Figure 17 Purge Test P-POD Back Plate

In order to accurately determine the static pressure inside the P-POD, it was necessary to prevent any of the high velocity flow exiting the gas inlet from coming into contact with the pressure sensor. To do this, a piece of cardboard was placed between the gas inlet and the pressure sensor, with the purpose of diffusing the high velocity flow. In this revision of the P-POD, there are gaps around access ports and between the door and collar. The door gap is not avoidable, as any attempts to cover it would hinder deployment. The gaps around the access ports can be covered simply by covering each 
entire side panel with tape. The first iteration of this tape method is shown below in Figure 18. Kapton tape was used and placed in parallel to the panel from the collar to the back plate. In an effort to prevent leakage through the different pieces of tape, each strip covers at least half of the previous strip of tape.

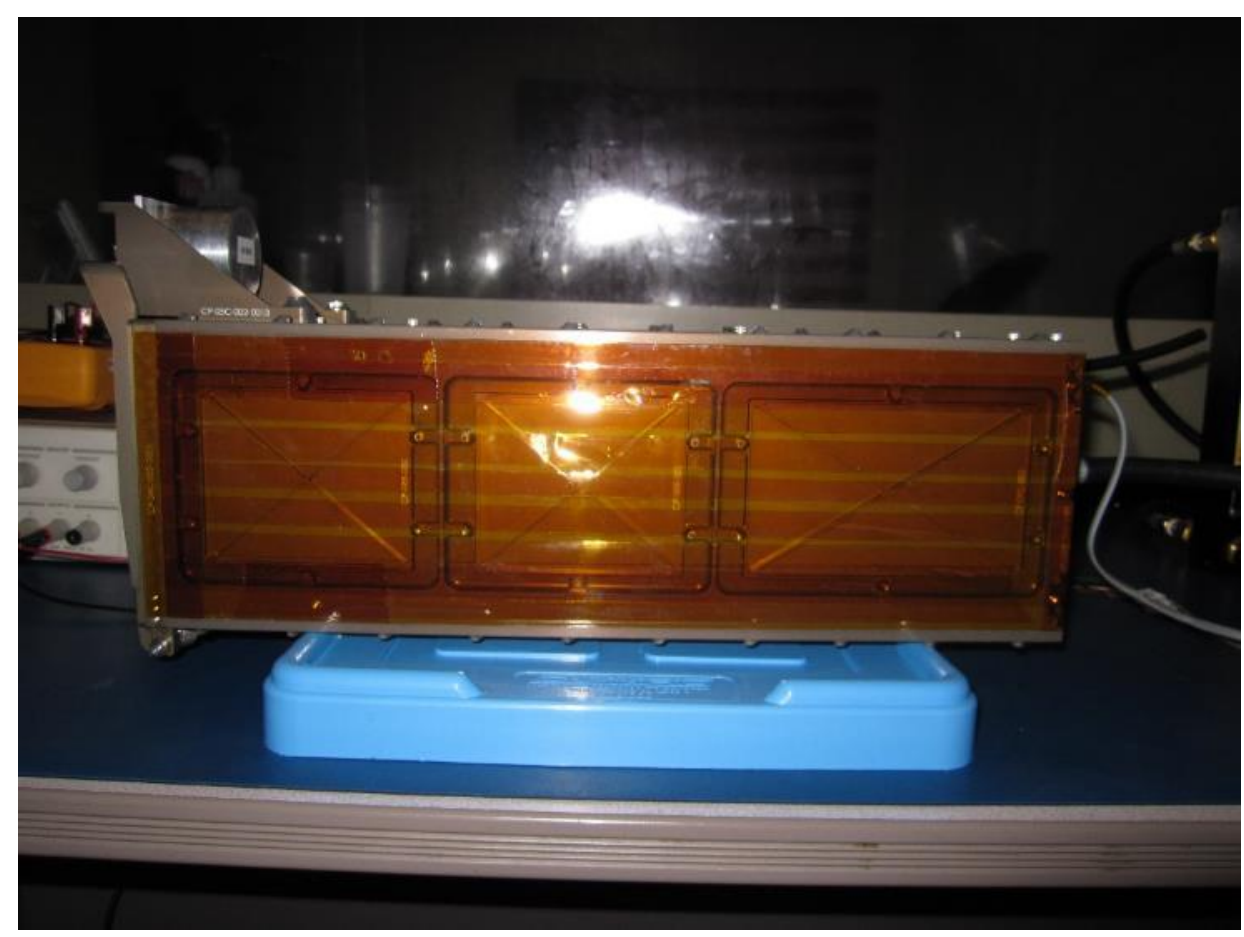

Figure 18 First Side Panel Taping Method

\section{First Gas Purge Test Run}

The first test run utilized the side panel taping method shown above, in the configuration shown above, with the P-POD mounted on its -Y bottom panel, to prevent the weight of the P-POD resting on the tape as it inflates, in flight like configuration. It is important to note that the door/collar assembly on this P-POD had previously undergone a severe qualification program, and was exhibiting an abnormally large door gap. Such a large door gap decreases the P-POD's ability to hold pressure and is a worse case test 
case. Inlet flow began at 1 SCFM, and was increased in increments of 0.5 SCFM every 5-10 seconds up to 8 SCFM. Results are shown below in Table 6. P-POD internal pressure was very low considering the range of low rates tested, which was attributed to the abnormally large door gaps. The door/collar assembly was immediately changed out for a different set with a much smaller door gap.

\section{Second Gas Purge Test Run}

The same test was repeated with the new door assembly, but this time at a flow rate of 5 SCFM, air began to escape the tape on the $+Y$ side of the $-\mathrm{X}$ side panel. These results are shown below in Table 7 . It is clear in the results that the tighter door gap made a profound difference, being the largest source of venting on the P-POD.

Table 6 Purge Test Data for Run 1

\begin{tabular}{|c|c|}
\hline Flow Rate (SCFM) & Internal Pressure (PSI) \\
\hline \hline 1.0 & 0.01 \\
\hline 1.5 & 0.02 \\
\hline 2.0 & 0.03 \\
\hline 2.5 & 0.05 \\
\hline 3.0 & 0.07 \\
\hline 3.5 & 0.09 \\
\hline 4.0 & 0.12 \\
\hline 4.5 & 0.16 \\
\hline 5.0 & 0.19 \\
\hline 5.5 & 0.24 \\
\hline 6.0 & 0.30 \\
\hline 6.5 & 0.37 \\
\hline 7.0 & 0.47 \\
\hline 7.5 & 0.57 \\
\hline 8.0 & 0.67 \\
\hline
\end{tabular}


Table 7 Purge Test Data for Run 2

\begin{tabular}{|c|c|}
\hline Flow Rate (SCFM) & Internal Pressure (PSI) \\
\hline \hline 1.0 & 0.03 \\
\hline 1.5 & 0.11 \\
\hline 2.0 & 0.21 \\
\hline 2.5 & 0.32 \\
\hline 3.0 & 0.46 \\
\hline 3.5 & 0.62 \\
\hline 4.0 & 0.80 \\
\hline 4.5 & 0.92 \\
\hline
\end{tabular}

\section{Third and Fourth Gas Purge Test Run}

Leakage through the tape is a clear indication that the taping method would need to be improved in order to allow for higher pressure, especially when under pressure for an extended duration. For the next test run, each flow rate was sustained for at least 30 seconds to measure the tape's ability to sustain pressure for an extended duration. At 3 SCFM after waiting about 30 seconds, the side panel tape began to leak again at the same location as the previous run, confirming the need for a more robust method of taping. Tape was added to both side panels in strips between the top/bottom panel screws. This new taping pattern is show below in Figure 19. The P-POD set back on its $-\mathrm{Y}$ bottom panel and testing was conducted in the same manner as the previous run, holding flow rates at steady state for at least 30 seconds. Results are shown below in Table 8 . 


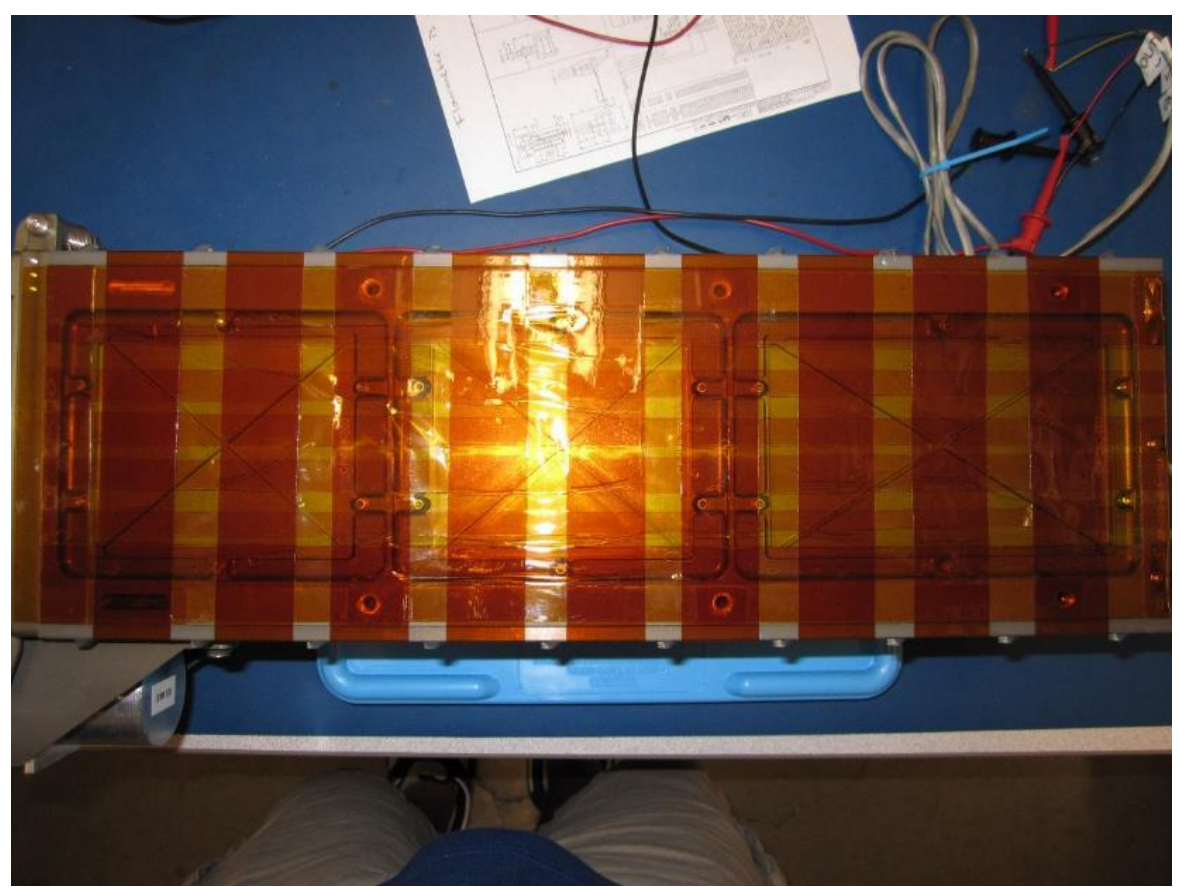

Figure 19 Reinforced Taping Pattern

Table 8 Purge Test Data for New Taping Method.

\begin{tabular}{|c|c|}
\hline Flow Rate (SCFM) & Internal Pressure (PSI) \\
\hline \hline 1.0 & 0.06 \\
\hline 1.5 & 0.13 \\
\hline 2.0 & 0.23 \\
\hline 2.5 & 0.34 \\
\hline 3.0 & 0.49 \\
\hline 3.5 & 0.65 \\
\hline 4.0 & 0.82 \\
\hline 4.5 & 1.01 \\
\hline
\end{tabular}

The setup held at just over 1 psi for 15-20 seconds prior to the Kapton tape along the $+\mathrm{X}$ side panel opened up, allowing air to escape. The internal pressure dropped down to a steady 0.6 psi immediately after. The leak occurred in areas along the edge of the 
tape not covered by the perpendicular strips of tape. An identical run was done again, and this time the tape opened while holding a constant 4 SCFM at 0.83 psi. These results are very consistent with previous test run. The modified P-POD's ability to hold pressure for a range of supplied flow rates was determined, and the results are summarized below in Figure 20.

Gas Purge Testing Results

On completion of these purge test runs, it was found that the P-POD can in fact hold a significant amount of positive pressure with a realistic flow rate supplied by the LV. For the current P-POD design, this requires that the $+\mathrm{X}$ and $-\mathrm{X}$ side panels be taped over to cover the gaps in the access port covers. The taping methods in this report were improved, and ultimately, the one-off design that flew utilized a sheet of Kapton film with its edges taped to the panel. With the Mk. IV P-POD which uses EMI gasketed access port covers, this taping will not be required as there will be no gaps in the access ports. This configuration will have very little risk of the seal failure that was seen with the air escaping through the tape.

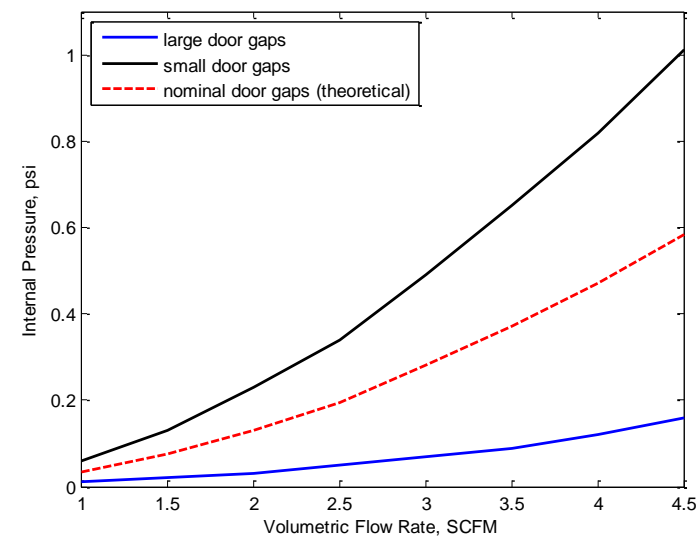

Figure 20 Internal Pressure vs Inlet Flow Rate Page 29 


\section{Mk. IV Back Plate Design}

The Mk. IV back plate comes standard with the ability to be modified to accommodate a similar purge setup as the one-off design that was flown on the ORS-3 mission. Instead of designing a separate back plate to be used for P-PODs with the purge capability, the standard back plate will be used, needing only 5 mission specific holes drilled, 4 of which being threaded holes. The back plate integrated with the purge access port is shown below in Figure 21. This design allows for quick modifications to the back plate to have it ready for a purge capable P-POD, rather than needing a whole part to be manufactured.
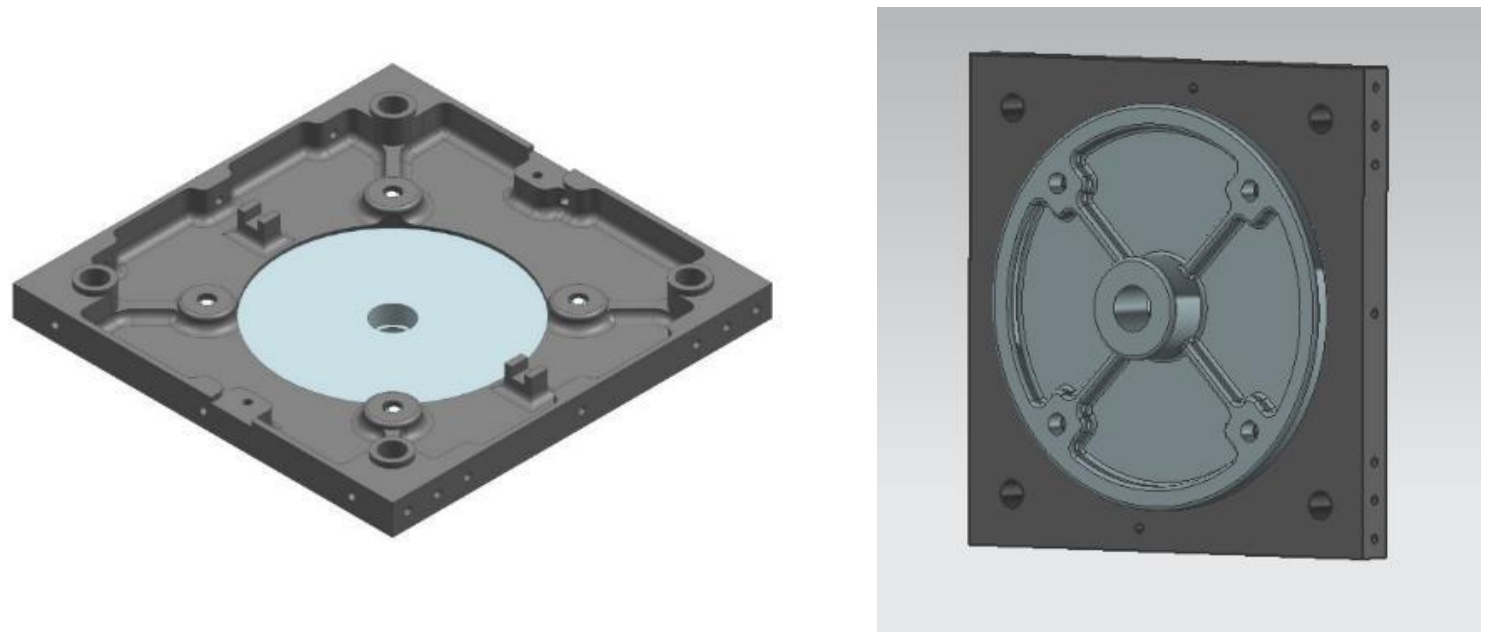

Figure 21 P-POD Nitrogen Purge Back Plate Design

\section{P-POD Power-On System}

The back plate interface used for the gas purge system can also be used to provide other specific capabilities, such as an electrical interface to the CubeSat payloads after CubeSat integration into the P-POD. The desire was to provide at least 3 circuits to support CubeSat power-on signal while integrated to LV, CubeSat battery charging while 
integrated onto the launch vehicle, and CubeSat system monitoring while integrated onto the launch vehicle. The first step was to explore a variety of interfaces, both wired and wireless, and determine the most feasible and effective method that would meet all requirements. The wireless options include using an inductive charging system, infrared radio, and standard Wi-Fi, while the wired option consisted of a standard differential line.

\section{Power-On System Wireless Options}

The following section describes wireless options for the Power-On system.

\section{Inductive Charging}

Inductive charging consists of high current running through tightly wound coiled wires which produces an electro-magnetic field, and then using a smaller concentric coil that picks up that electro-magnetic field, and in turn produces current. The primary coil would be housed within the pusher plate, while the secondary, receiving coil would be protruding from the CubeSat. This charging method requires no physical contact, and can also double as a wireless data transfer method, but can only support data rates up to $300 \mathrm{bps}$, which are insufficient for basic health check and very noisy. Therefore, the inductive charging system would require other wireless interfaces to send power-on signal and monitor CubeSat systems. Additionally, the DC power from the LV requires a $\mathrm{DC}$ to AC inverter for the primary coil, and the current coming from the secondary coil needs to be rectified in order to provide a DC charging current to the CubeSat batteries. At every stage there are inefficiencies, leading to an inefficient charging system as a whole. 


\section{Infrared Signal}

Using an infrared signal to power-on the CubeSat would be a very simple, effective method of doing so, but any systems monitoring would require significant interfacing electronics. While this is possible, it as an increasingly complex method of monitoring systems, and requires direct line of sight. Infrared is also not an option for battery charging, meaning it would have to be used in conjunction with the inductive charging system.

\section{Wi-Fi System}

At first glance, Wi-Fi is the best wireless method for systems monitoring, with high data rates, support for several CubeSats, and no need for line of sight. Unfortunately, Wi-Fi cannot be used to charge CubeSat batteries. Additionally, using WiFi requires that the Wi-Fi module on the CubeSat be powered on, which prevents it from being used to signal the CubeSat to turn on. In the end, none of these wireless systems can meet all of the requirements on their own. In fact, all three of them are needed in order to meet the requirements. Using three separate systems is very complex, and not the best option.

\section{Wired Power-On System}

Using a simple differential pair interface can meet all requirements through a single connector. The only downside is a physical interface is required, but there are existing connector solutions that require little to no force to disengage. A connector such as this placed into the P-POD inside of the pusher plate could mate to the other connector half, attached to the CubeSat. An example of this setup is shown below in Figure 22. 


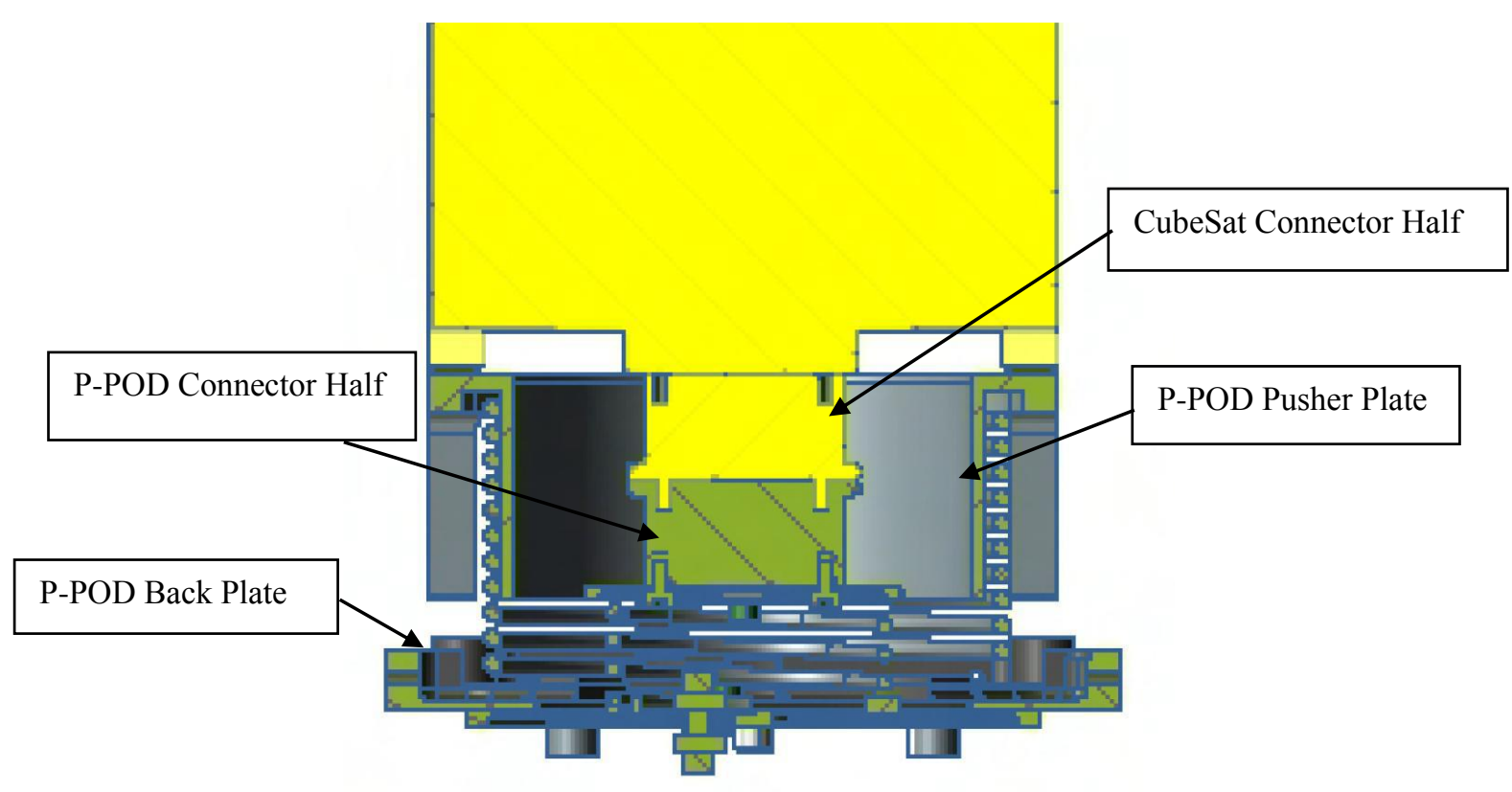

Figure 22 P-POD Power-On System Configuration.

The configuration shown above is one of the direct contact wired options. Another option was to utilize a collection of items such as springs and pin contacts in order to achieve the same goal without using a commercial off-the-shelf connector.

\section{In-House Conical Spring System}

An example of this setup is shown below in Figure 23. The conical springs and the circular pads shown are conductors, and the pins serve as guides to ensure the conical spring does not slip off its designed location, ensuring contact. This setup could be put together for relatively low cost but there were some concerns. For instance, it would be difficult to provide industry approved shielding to each of the conical springs without interfering with their function. This system would also require a significant amount of assembly, which makes workmanship errors more likely and prolongs build times. It 
does however offer no hindrance to deployment, and is simple enough in its function such that there is little chance for disruptions in continuity.

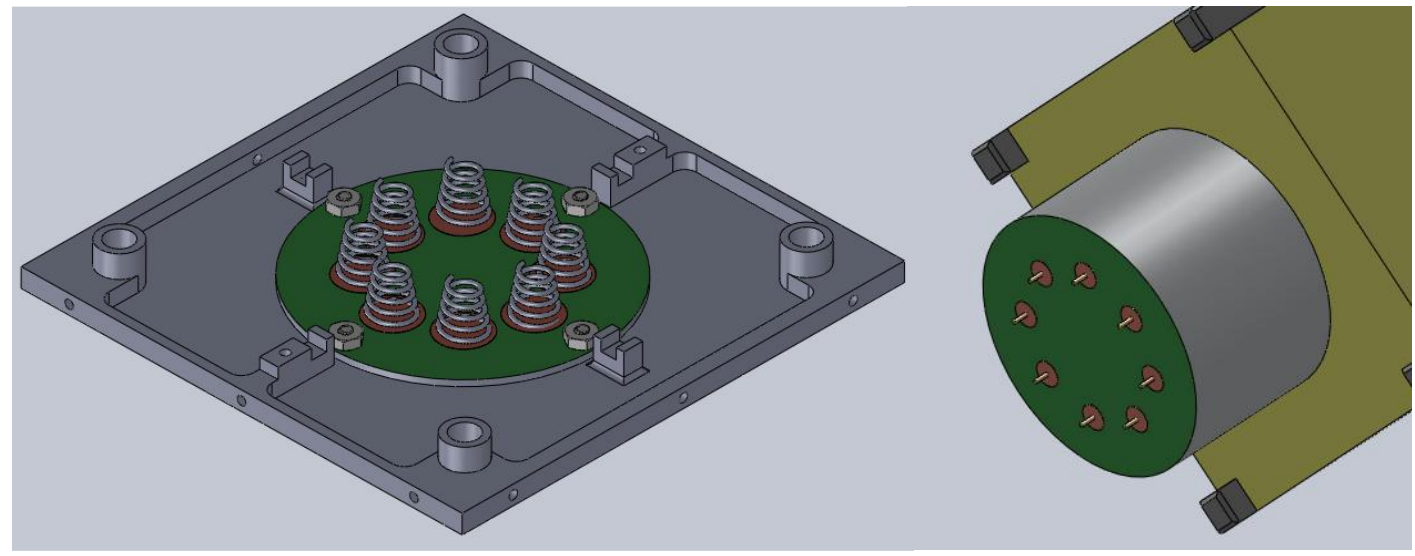

Figure 23 Wired Interface Without Connector.

\section{Commercial Separation Connector Solutions}

Using a commercial off the shelf connector designed for low or zero-force separation offers many benefits. It looks good to launch providers and CubeSat payloads alike, to use industry proven electrical connectors. Two units were investigated and each had its own set of advantages and disadvantages. The first was a Glen-Air MIL-STD38999 connector, such as the one shown below in Figure 24. This robust connector has flight heritage and can support up to 37 electrical contacts, which is restricted by the connector diameter. Even with smaller sets of contacts, the connector design is bulky and would be a very tight fit inside the P-POD, which could make internal circuit wiring difficult. The 22D AWG pins can support 5 Amps, which is much more than a CubeSat would draw for battery charging ( 1 Amp). The other connector is a separation connector used by Planetary Systems Corporation (PSC) on their Lightband spacecraft separation rings. These connectors are essentially an array of pogo-pins aligned in a 
shielded housing. They have flight heritage as well, but are less resistant to random vibration. This presents a risk for electrical intermittencies during launch, but this was seen as not a driving issue as the function of these circuits will not be affected by intermittencies in the event that they do occur. This connector is shown below in Figure 25.

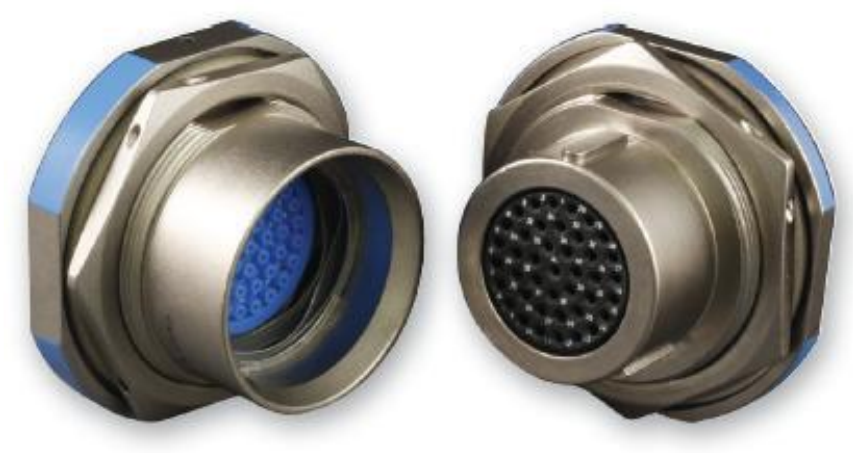

Figure 24 Glen-Air Zero Ejection Force Connector
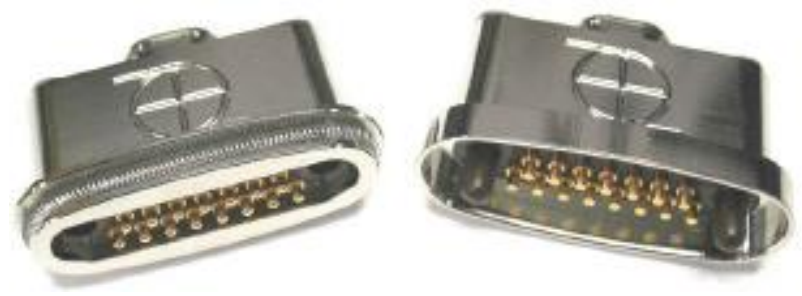

Figure 25 Planetary Systems Corporation Separation Connector

Power-On System Down-Selection

The following section describes the series of trade studies to determine the best Power-On system design. 
Wired vs. Wireless

The initial trade study to determine the best method for the P-POD to CubeSat electrical interface was primarily focused on the wireless methods versus using a standard wired interface. Several trade metrics were considered. The first being how well it would satisfy the design requirements. Most of the wireless cases came up short in this category as they each required another wireless method to capture all of the requirements. The next category was a measure of the risk of hindering deployment, and all of the options can be made to have almost no effect on deployment. Another metric was how well each trade could maintain continuity. Having a wired connection was determined to be most reliable through the use of flight proven connectors. The complexity of the system was also looked at, primarily from the standpoint of how many components are required. The inductive charging option scored very low in complexity due to it requiring more than one power converter. Finally, the ease of assembly, integration, and testing was examined to minimize the amount of time and risk associated with those three processes. The first three metrics were weighted twice as heavily due to their direct impact to the design requirements and P-POD functionality. The trade summary is shown below in Table 9. By fair margin, the wired differential line option seemed to be the most favorable option. It satisfies all design requirements while maintaining reliable deployment and, through the use of flight proven separation connectors, provide a reliable electrical interface. It is also the simplest option, as the primary function simply relies on a connector rather than extra electrical modules and inductor coils. 
Table 9 P-POD Electrical Interface Trade Summary (1-5, 5 is best)

\begin{tabular}{|l|c|c|c|c|c|c|}
\hline \multicolumn{1}{|c|}{ Signal } & $\begin{array}{c}\text { Satisfies } \\
\text { Requirements } \\
\text { Weight }=2\end{array}$ & $\begin{array}{c}\text { Deployment } \\
\text { Reliability }\end{array}$ & $\begin{array}{c}\text { Continuity } \\
\text { Reliability }\end{array}$ & Complexity & $\begin{array}{c}\text { Ease of } \\
\text { Assembly } \\
\text { and I\&T } \\
\text { Weight }=1\end{array}$ & Total \\
\hline $\begin{array}{l}\text { Inductive } \\
\text { Charging }\end{array}$ & 3 & 5 & 4 & 1 & 4 & 29 \\
\hline Infrared & 3 & 5 & 4 & 4 & 4 & 32 \\
\hline Wi-Fi & 2 & 5 & 4 & 4 & 5 & 31 \\
\hline $\begin{array}{l}\text { Differential } \\
\text { Line }\end{array}$ & 5 & 5 & 5 & 5 & 4 & 39 \\
\hline
\end{tabular}

\section{Wired System Trade Study}

The next step was to narrow down the wired options to determine which option to proceed with. The three options examined were the in-house option described above that utilized conical spring contacts, as well as the two separation connectors. In this case, deployment and continuity reliability, and complexity were considered, as well as cost and area of interface required on the CubeSat. None of the wired options considered presented a risk for deployment. The conical spring trade and PSC connector both contain spring loaded contacts, which can present a risk for continuity intermittencies. Both the Glen-Air and PSC connectors are simple, off the shelf connectors, and do not have the inherent complexity in putting together the circuit boards required to accomplish what is proposed in the conical spring trade. Both the conical spring system and the Glen-Air connector are relatively wide interfaces, that would require use of a large portion of the $-\mathrm{Z}$ face of the CubeSat. The PSC connector on the other hand has a much slimmer profile, and is a simpler connector so it takes up much less space. Unfortunately, because PSC is a smaller company, their connector is several times the cost of the Glen- 
Air connector. A summary is shown in Table 10 below. All three options were very closely ranked, but the conical spring system was dropped due to the difficulty in adequately shielding all of the contacts, along with lack of flight heritage. The decision made was to push forward with the PSC connector, with the stipulation that the final system could be adapted for use with the Glen-Air connector with minimal adjustment.

Table 10 Wired Interface Trade Summary

\begin{tabular}{|c|c|c|c|c|c|c|c|}
\hline Trade & $\begin{array}{c}\text { Deployment } \\
\text { Reliability }\end{array}$ & $\begin{array}{c}\text { Continuity } \\
\text { Reliability }\end{array}$ & Complexity & $\begin{array}{c}\text { Area of } \\
\text { Interface (on } \\
\text { CubeSat) }\end{array}$ & Cost & $\begin{array}{c}\text { Ease of } \\
\text { Assembly } \\
\text { and I\&T }\end{array}$ & Total \\
\hline \hline $\begin{array}{c}\text { Conical } \\
\text { Spring }\end{array}$ & 5 & 5 & 2 & 2 & 5 & 2 & 21 \\
\hline Glen-Air & 5 & 5 & 5 & 2 & 3 & 2 & 22 \\
\hline PSC & 5 & 4 & 5 & 4 & 1 & 4 & 23 \\
\hline
\end{tabular}

Power-On System Design

The Power-On system consists of both a mechanical interface and an electrical interface to the CubeSat. Additionally, CubeSat's that desire access to these capabilities must be designed to a standardized interface, to mitigate any recurring engineering costs.

\section{Mechanical System Design}

The first consideration when designing this electrical interface, was that it needed to accommodate a range of positions in all three axes, to account for tolerance allowances. The determined solution was to mount the P-POD half of the connector to a small aluminum plate that was attached to the back plate through a spring. This would provide a loose enough interface such that the connector could move to accommodate the CubeSat connector half in whatever position it is in during integration. Additionally, it would allow the connector to translate should the CubeSat shift in the P-POD, preventing 
any shear load or bending moment on the CubeSat connector. The connector spring would also provide allowance in the $\mathrm{Z}$ axis, and it would aid in deployment by providing an extra kick. The next feature incorporated into the design, was compatibility with the back plate purge interface so that the same back plate could be used for P-PODs with the electrical pass through capability. Therefore, a back plate cover was designed that would accommodate the connector spring and exterior connector. A Micro-D style connector was chosen for the exterior connector because of its small surface area and volume requirements. The cover was designed in two halves, one half mounted the exterior connector and connector spring and the other simply supported the spring and closed the access port. This would allow for an access point in the back plate during and after integration, without disturbing the connector spring or internal wiring. An exploded view of this system is shown below in Figure 26.

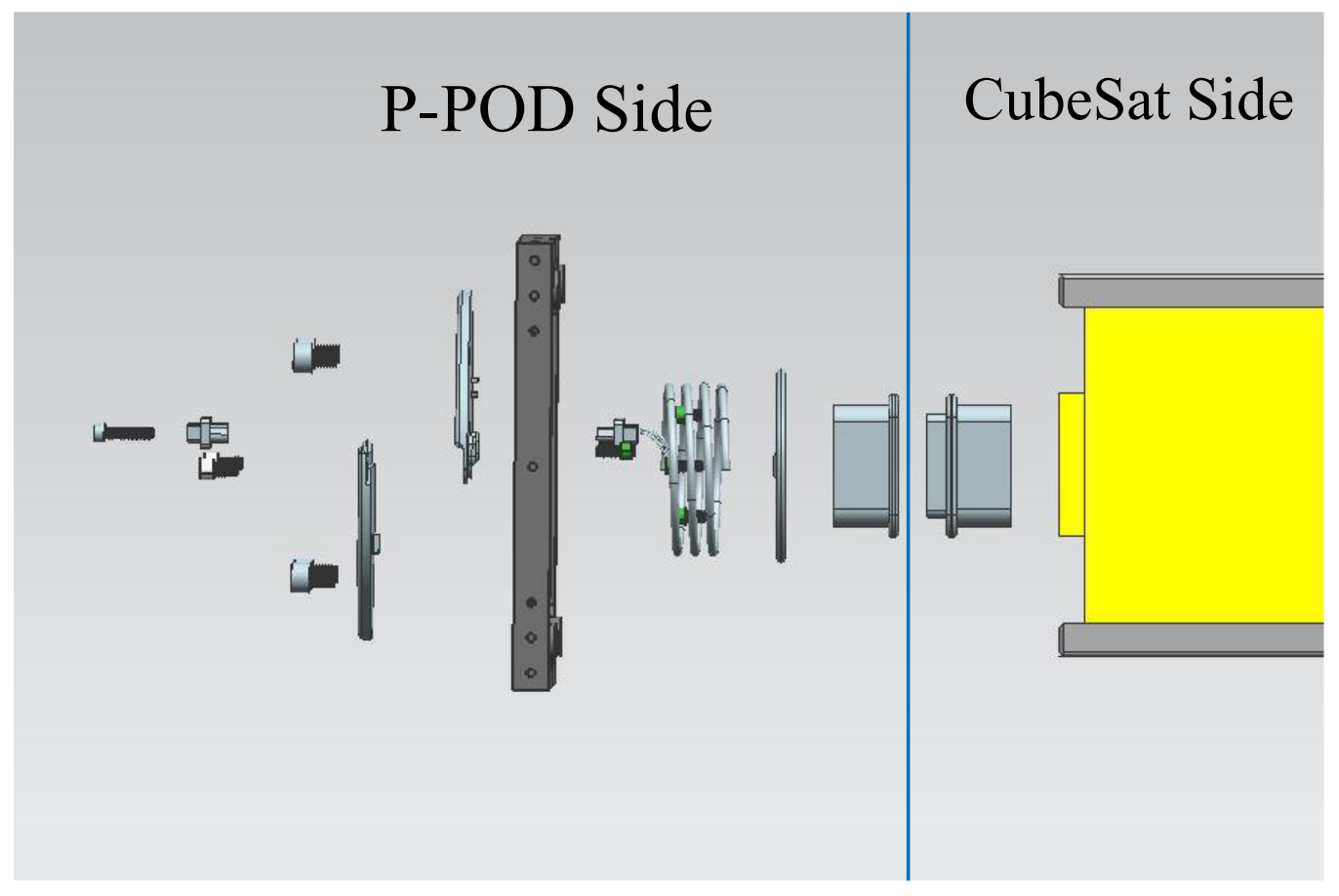

Figure 26 Separation Connector Exploded View.

Page 39 
The CubeSat is to mount its connector half in a standardized location and orientation, and that is the only requirement levied on the CubeSat. The rest of the interface is on the P-POD side. Its connector half is mounted to the spring perch, which is fixed to the spring through the use of Tefzel loop straps. Tefzel is a low outgassing plastic with flight heritage and conforms to NASA outgassing standards. The spring is then mounted to one of the access port cover halves, which is mounted to the back plate with size 8-32 screws. The separation connector is wired to the fixed Micro-D exterior connector with enough slack to allow it full range of motion. Then the second half of the back plate access port cover is attached to the back plate with size 8-32 screws, and then two retaining screws are installed in the two corners of the second access port cover that overlap the first cover. The Micro-D external connector was used instead of a standard MIL-DTL-38999 connector because of space limitations. For launch vehicle integration, it is usually preferred to have a MIL-DTL-38999 connector as the interface to the launch vehicle due to its robust method of attachment. In this case a harness of specified length may be required, with the Micro-D connector to the P-POD on one side, and a 38999 style connector on the LV side.

\section{Electrical System Design}

The PSC connector has 15 pins, which is more than the minimum required. To meet the basic design requirements, nine pins would be needed. The battery charging circuit requires a 5V DC line and ground. Power-on signal would require two differential lines for noise mitigation and a ground line for a total of three lines. Systems monitoring would require two differential lines, a ground, and a transmit/receive line for a total of four lines. Having an extra six pins available can possible support a redundant charging 
circuit, more systems monitoring circuits, or a redundant power-on signal circuit. There is enough room to fit two of these connectors if for some reason the payload requires more than 30 lines. A circuit drawing of the base system is shown below in Figure 27.

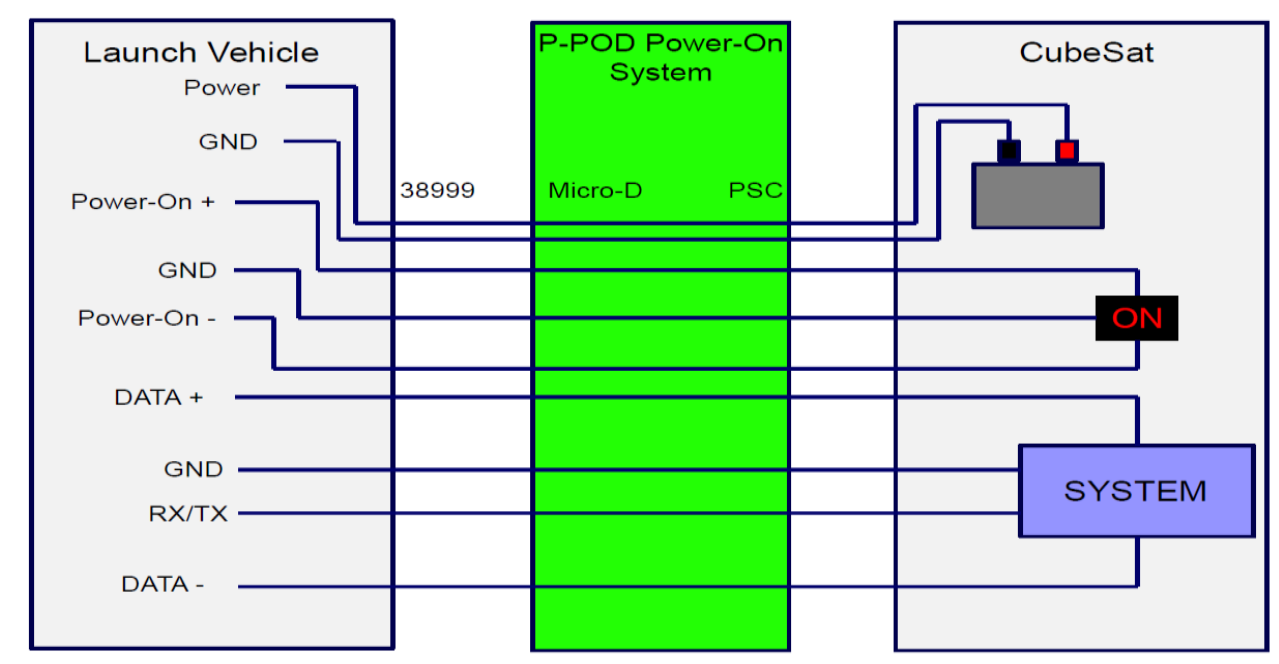

Figure 27 P-POD Power-On System Circuit Drawing.

\section{CubeSat Interface Specification}

The standardized CubeSat interface will consist of the CubeSat connector half mounted to the center of the $-Z$ face of the CubeSat, such that the back plane of the connector housing is flush with the plane created by the $-\mathrm{Z}$ rail standoffs. The reason this location was chosen was that it is important to maintain the CubeSat's useable volume, employing as few limitations to the CubeSat as possible. Connector orientation is less critical, but must be the same every time, so it will required that the connector be mounted such that the long axis of the connector is along the $\mathrm{Y}$-axis of the CubeSat. This interface specification is show below in Figure 28. 

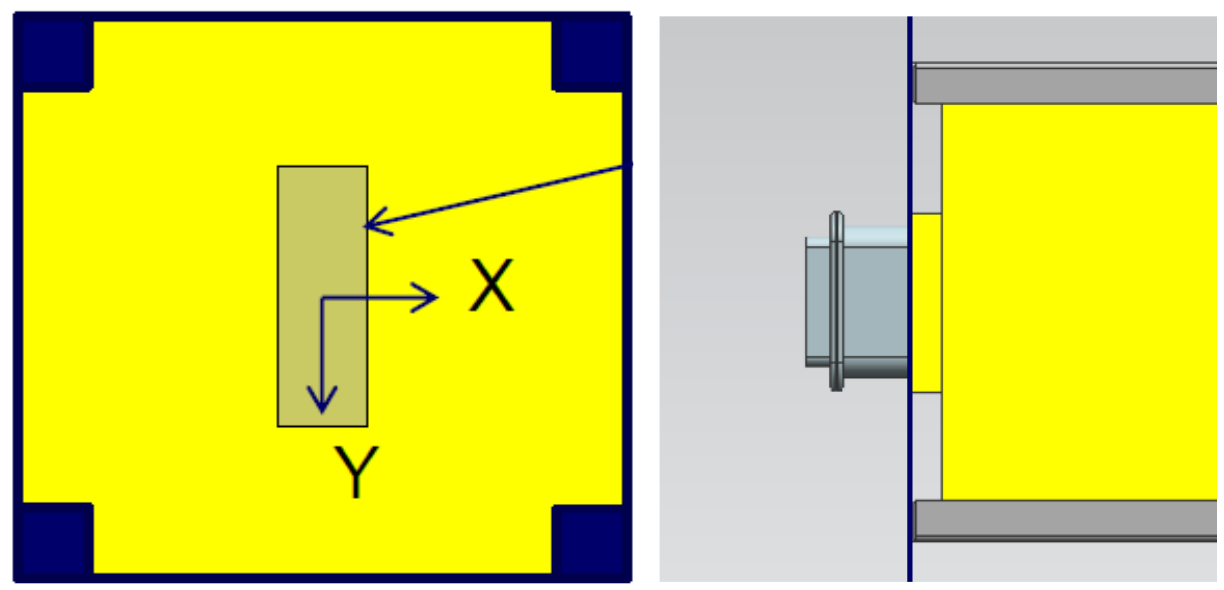

Figure 28 CubeSat Connector Interface

\section{Power-On System Structural Analysis}

The next step was to show that the design was structurally sound. This was not a significant concern given the loads it was going to see, but it is still important to show launch providers that the entire P-POD is structurally sound and that the access port halves will not gap. A conservative load case was used, assuming the entire load of the connector spring was placed on the access port half that it was mounted too. Additionally, the spring was assumed to be compressed twice as much as expected, a total of 0.5 ", which would produce a force of $14 \mathrm{lbf}$. Added to the plate was a $100 \mathrm{~g}$ gravity load in the Z-axis, which is higher then any qualification load the P-POD would see. The access port cover was fixed at its mounting holes, but the supporting spar from the second access port spar was ignored to get a worst case load scenario. The FEM analyzed showing constraints and loads is shown below in Figure 29, with Deflection and Stress plots shown in Figure 30. Maximum deflection for the specified load case was 0.004 inches, as shown. The maximum stress was seen on the ribs near the mounting holes, and was calculated to be 5616 psi. It is necessary that the Margin of Safety be calculated for 
clarity. Margin of safety is calculated as shown in $\boldsymbol{M S}=\frac{\boldsymbol{S}_{a}}{S}-\mathbf{1}$

Equation 2.

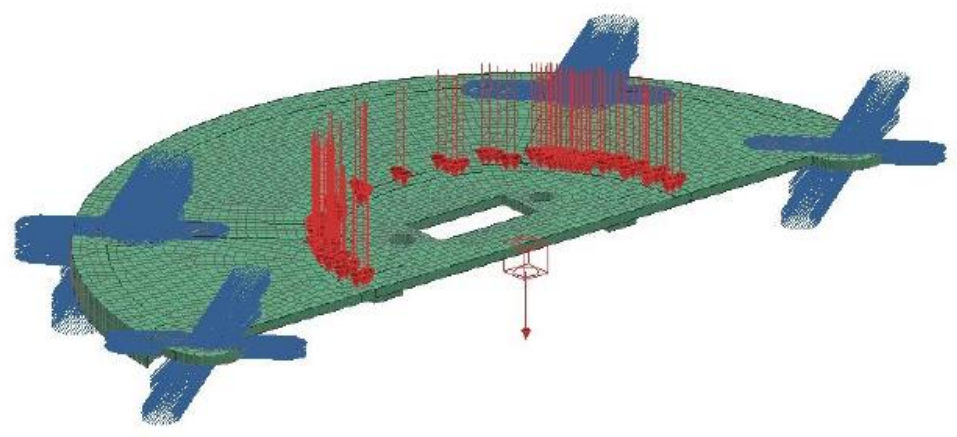

Figure 29 Power-On Access Port FEM

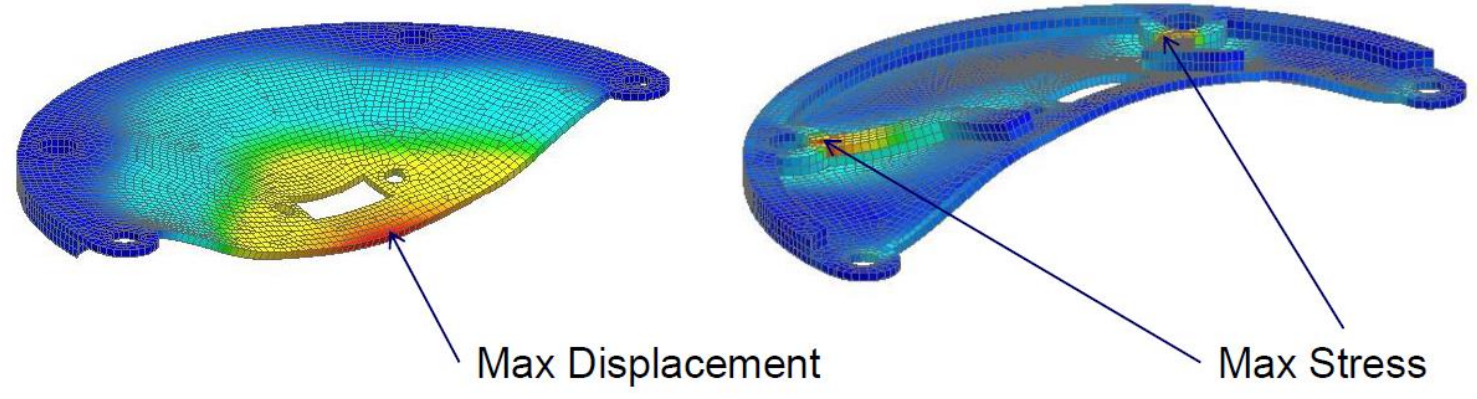

Figure 30 Power-On Access Port FEA Results

$$
M S=\frac{s_{a}}{S}-1
$$

Equation 2

In this case, $S_{a}$ is the material allowable stress, or yield stress when doing yield analysis, and $S$ is the stress seen from the applied load. This part exhibited an extremely 
high margin of safety of 10.24 , while the acceptable margin of safety for yield strength analysis is greater than 0.0 .

The next analysis case was to show the effect of the back plate changes on the fundamental frequency of the P-POD. The concern was that cutting a hole in the back plate would cause a reduction in stiffness that would lower the fundamental frequency of the P-POD. The outer walls of the back plate were assumed to be fixed, and the resulting displacement plot of the $1^{\text {st }}$ mode of the new back plate is shown below in Figure 31. As

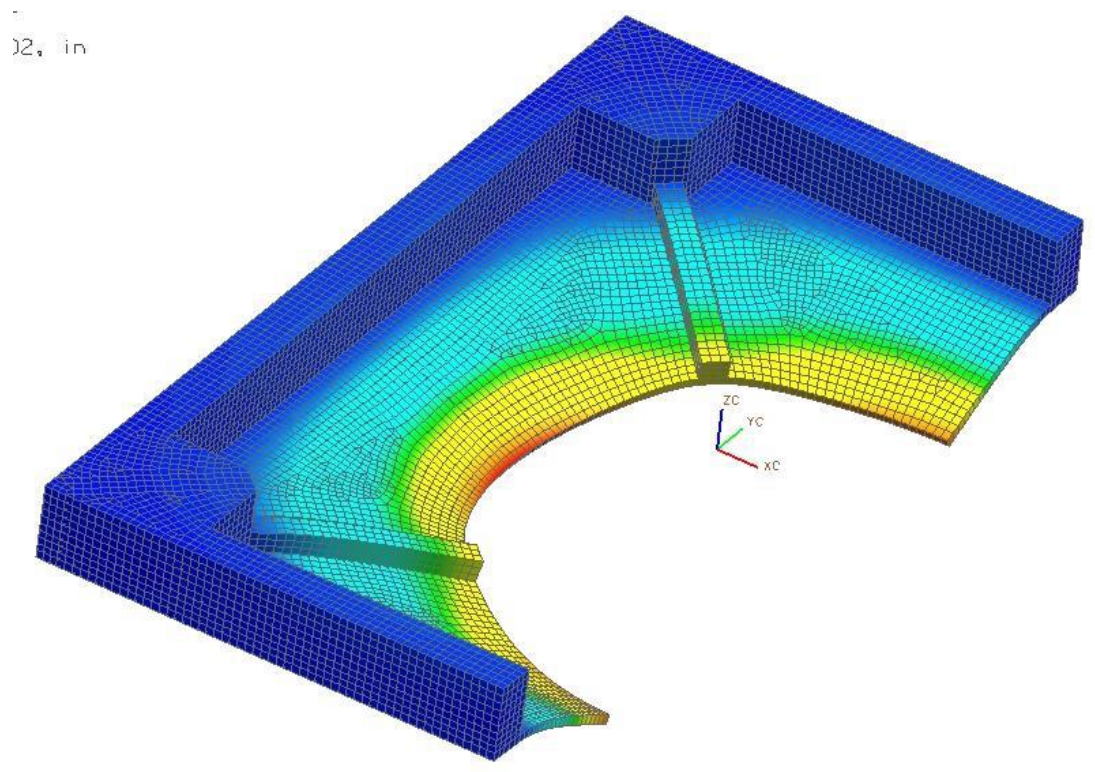

Figure 31 1st Mode of Power-On/Purge Style Back Plate

expected a basic panel mode appeared as the first mode. However, instead of reducing in natural frequency, the lack of mass at the center of the back plate increased the first mode substantially. Comparison frequencies from the Mk. III Rev. E back plate, and the Power-On/Purge back plate are shown below in Table 11. For reference, the P-PODs 
natural frequency is around $120 \mathrm{~Hz}$, therefore it was concluded that the modifications to the back plate would not significantly effect the natural frequency of the P-POD.

Table 11 Back Plate Fundamental Frequencies

\begin{tabular}{|c|c|c|}
\hline Mode & Standard & Power-On/Nitrogen Purge \\
\hline \hline 1st & $1213 \mathrm{~Hz}$ & $2538 \mathrm{~Hz}$ \\
\hline 2nd & $3215 \mathrm{~Hz}$ & $3095 \mathrm{~Hz}$ \\
\hline 3rd & $4301 \mathrm{~Hz}$ & $4890 \mathrm{~Hz}$ \\
\hline
\end{tabular}

Power-On Preliminary Design Conclusion

In summary, it was conluded that the Power-On system was structurally sound and was capable of meeting the design requirements. The system can accommodate a wide tolerance range and does not impose any constrain on CubeSat movement once integrated to the P-POD. The system was primarily designed for 1 15-pin PSC separation connector, but can easily be adapted to accommodate two of these connectors, or the larger and more versatile Glen-Air connector design. The system can support battery charging with a $5 \mathrm{~V}, 1 \mathrm{~A}$ power supply, as the connectors are limited to $3 \mathrm{~A}$ per contact which is in excess of the 1A standard. The preliminary design for this system is complete, but has not been put into production at this time. 


\section{CHAPTER IV: P-POD MASS REDUCTION}

The P-POD has undergone several design iterations over the years in an effort to improve its capabilities. The early P-PODs exhibited high stress concentrations near fasteners due to very low edge-to-hole distances. Between the P-POD Mk. III Rev. C and the Mk. III Rev. E, every fastener hole was evaluated, and most were modified to provide thicker material at through-holes and more distance from the panel edges to fastener holes. Industry advised the use of the 1-diameter rule of thumb. This guideline suggests using the major diameter of the hole to determine threaded and through-hole thickness, as well as distance from the panel edge to the edge of the fastener hole. Additionally, because helical inserts are used in the mounting holes of the P-POD, there is a recommended insert length from the insert manufacturer for a given fastener material/load. Because high strength stainless steel screws are used frequently, the PPOD was designed to use helical inserts with lengths of 1.5 times the major diameter of the 10-32 mounting screws, or 0.285 inches. This drives the mounting region of each $\mathrm{P}-$ POD panel to be unnecessarily thick. All of these changes increase the robustness of the P-POD, but also have resulted in a non-trivial increase in mass. The objective of the mass reduction pass over the P-POD was to dial back some of the mass gains without introducing any weak points in the structure. Finally, some small changes that were overlooked in the past were corrected.

\section{P-POD Design Loads}

In order to determine the loads for analysis, the random vibration environment on the NPSCuL, integrated to an Atlas V launch vehicle, was considered. The launch 
vehicle specifies levels at the NPSCuL interface, but these are not appropriate levels to use for P-POD analysis. A P-POD has been tested in this configuration before, so it was possible to use the results from that test to determine the levels experienced by the PPOD at the P-POD to NPSCuL mounting interface. The corresponding Acceptance levels are shown below in Figure 32. In order derive a static load case from random vibration load case, the vibration response spectrum (VRS) tool was used. The VRS function

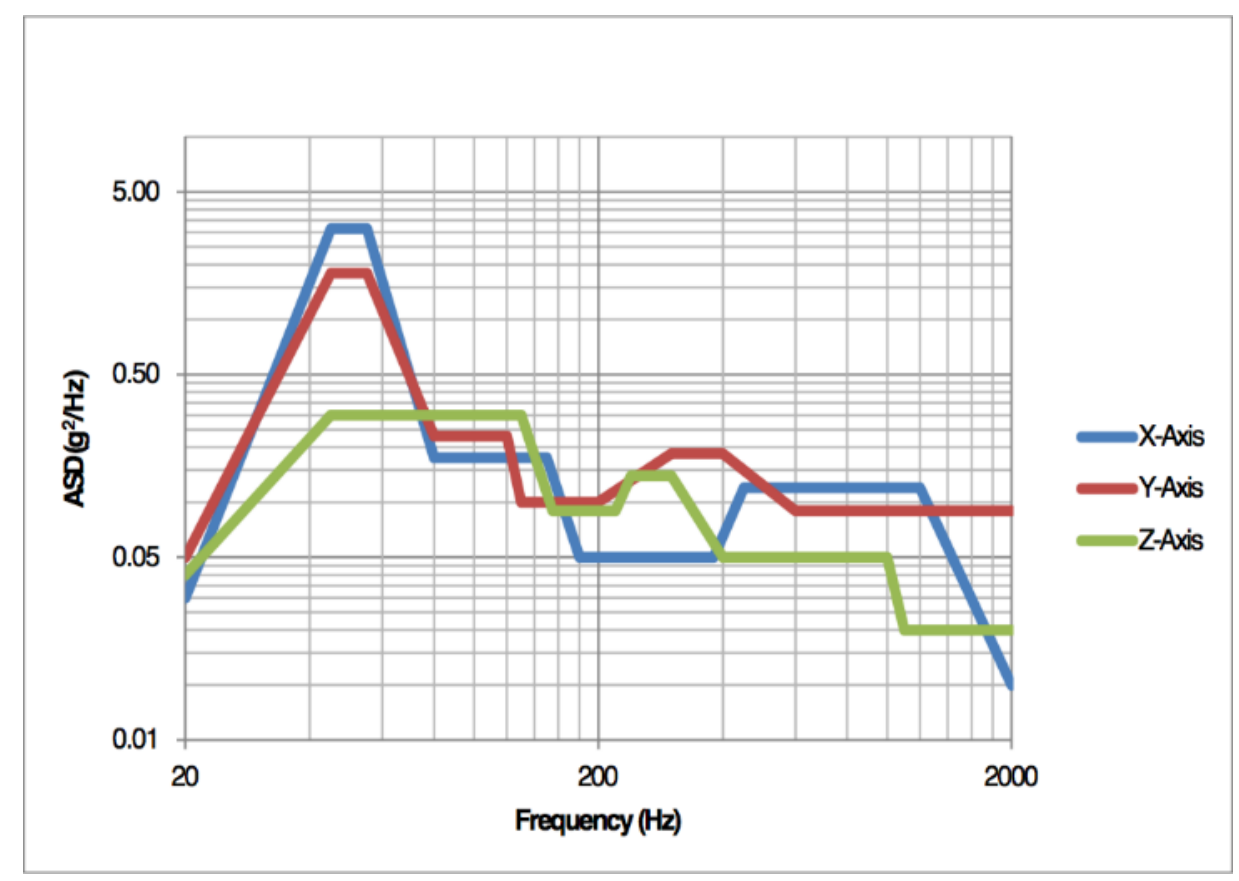

Figure 32 NPSCuL Acceptance Loads at P-POD Interface

determines the root-mean-square acceleration $\left(\mathrm{g}_{\mathrm{rms}}\right)$ response of a system to a base input, such as random vibration. There are two equations that can be used in order to determine the appropriate static acceleration, Miles Equation and a more general equation. Miles Equation is a simpler calculation, but is only applicable when the power spectral density levels are flat for an octave on either side of the fundamental frequency of the system. 
The General Approach was used because of its non-restrictive nature, and because it was going to be solved using Matlab. The general equation, as a function of natural frequency and damping ratio, is show below in $\ddot{x}_{G R M S}\left(f_{n}, \xi\right)=$

$$
\begin{aligned}
& \sqrt{\sum_{i=1}^{N} \frac{\left\{1+\left(2 \xi \rho_{i}\right)^{2}\right\}}{\left\{\left[1-\rho_{i}\right]^{2}+\left[2 \xi \rho_{i}\right]^{2}\right\}}} \widehat{\boldsymbol{Y}}_{A P S D}\left(f_{i}\right) \Delta f_{i}, \rho_{i}=\frac{f_{i}}{f_{n}} \quad \text { Equation } 3 \text { (Irvine 2009). } \\
& \ddot{x}_{G R M S}\left(f_{n}, \xi\right)=\sqrt{\sum_{i=1}^{N} \frac{\left\{1+\left(2 \xi \rho_{i}\right)^{2}\right\}}{\left\{\left[1-\rho_{i}{ }^{2}\right]^{2}+\left[2 \xi \rho_{i}\right]^{2}\right\}}} \widehat{Y}_{A P S D}\left(f_{i}\right) \Delta f_{i}, \rho_{i}=\frac{f_{i}}{f_{n}} \quad \text { Equation } 3
\end{aligned}
$$

In this equation, $f_{i}$ is frequency (x-axis of ASD plot) and $\widehat{Y}_{A P S D}$ is the base input level (y-axis of ASD plot). The input level is given, but the damping ratio and natural frequency are needed. The best method of determining these values is from previous tests of a similar system. The natural frequency, $f_{n}$ can be identified by examining the response of a previous test, and identifying the first mode in each axis of testing. The damping ratio, $\xi$, can be determined from the Q-factor.

The Q-factor is the ratio of energy stored to the energy dissipated per cycle. It relates to $\xi$ by the equation,

$$
Q=\frac{1}{2 \xi}
$$

The Q-factor is determined through testing, by using the half-power bandwidth of the response peak at the fundamental frequency. In this instance, the same $\mathrm{Q}$ is described as,

$$
Q=\frac{f_{n}}{\Delta f}
$$

Equation 5 
Where $\Delta f$ is the bandwidth at the half-power point of the first mode peak in the vibration response data from previous testing. The Q-factor was determined through testing of a previous revision of the $\mathrm{P}-\mathrm{POD}$, because the structure is so similar. Once $\mathrm{Q}$ is determined, $\xi$ is determined from solving the first $\mathrm{Q}$ equation. (Irvine 2005)

Additionally a 3-sigma factor was applied to the output $\mathrm{g}_{\mathrm{rms}}$ value, as there is a $99.73 \%$ chance that the static load will reside inside the \pm 3 -sigma limits of the $g_{r m s}$ output. For this case, the inputs to the VRS function are shown below in Table 12, along with the output load.

Table 12 VRS Input Values and Output Loads

\begin{tabular}{|c|c|c|c|}
\hline Axis & Fundamental (Hz) & Q & Load (g's) No F.S. \\
\hline \hline X & 130 & 8.67 & 60.2 \\
\hline Y & 260 & 3.71 & 50.0 \\
\hline$Z$ & 165 & 4.13 & 40.1 \\
\hline
\end{tabular}

The worst case was taken for the $\mathrm{X}$ - and $\mathrm{Y}$-axis loads, to account for mounting on either side of the P-POD. Additionally, a conservative static launch vehicle acceleration of $8.75 \mathrm{~g}$ 's (supplied by ULA) was applied to all 3 axes. The load calculated so far is for Acceptance levels. In order to obtain the Qualification by analysis levels, the Acceptance loads need to be multiplied by a no-test factor of safety of 1.6, as per the Launch Services Program Program Level Requirements Document (LSP-REQ-317.01). The resulting loads are shown below in $\boldsymbol{L}_{x, y}=(\mathbf{6 0 . 2}+\mathbf{8 . 7 5}) \times \mathbf{1 . 6}=\mathbf{1 1 0 . 3} \mathrm{g}$

Equation 6 and $\boldsymbol{L}_{z}=(\mathbf{4 0 . 1}+\mathbf{8 . 7 5}) \times \mathbf{1 . 6}=\mathbf{7 8 . 2} \mathrm{g}$

Equation 7.

$$
L_{x, y}=(60.2+8.75) \times 1.6=110.3 g
$$




$$
L_{z}=(40.1+8.75) \times 1.6=78.2 g
$$

Equation 7

The resulting static acceleration calculated was applied to a CubeSat payload mass of $6 \mathrm{~kg}$, as the upper bound of expected CubeSat masses at this point in time. Additionally, for calculations in the Z-axis of the P-POD, the total spring force, including the P-POD main spring and back plate spring plungers, of $67.6 \mathrm{lbf}$ was applied to components loaded in the Z-axis, primarily the P-POD Door, NEA bracket, and collar.

\section{P-POD Mk. IV Part Design and Analysis}

This section describes the part by part design changes and analysis to show that the P-POD Mk. IV will survive the expected loads.

P-POD Mk. IV Top Panel

The P-POD top panel is never used as a mounting surface but is still a structural component in contain CubeSat loads. Additionally, the release mechanism bracket attaches to the Top Panel. The P-POD Mk. III Rev. E Top Panel is shown below in Figure 33. The four Bracket mounting holes at the top were moved outward 0.150 inches in order to accommodate through holes on the Bracket that are properly centered, as the previous design had off center through-holes. The two ribs running down the length of 


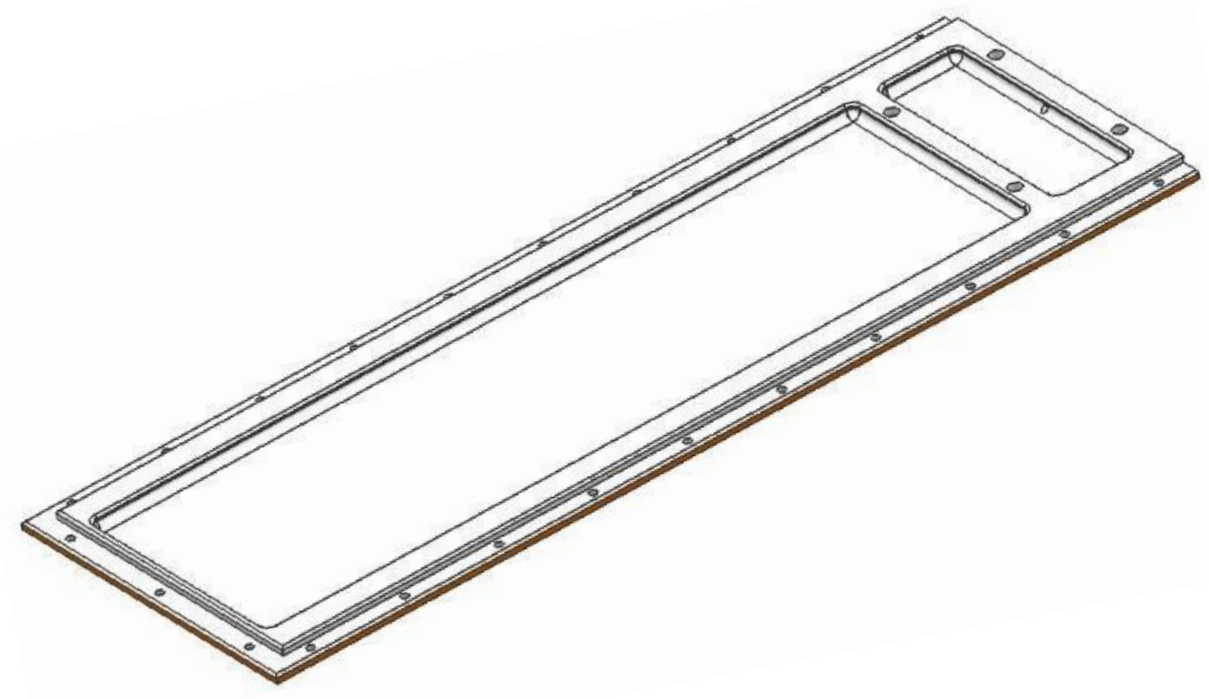

Figure 33 P-POD Mk. III Rev. E Top Panel

the panel are currently sized to accommodate the mounting holes with both adequate width and thickness. These ribs were increased for Rev. E but are suspected to be excessive for the design intent of the Top Panel. Additionally, as discussed in Chapter II, a venting hole array designed to shield EMI/RFI was added as a non-standard mission specific case. The resulting design is shown below in Figure 34. The panel has been reduced to the minimum it needs to be. The ribs are now considerably thinner, but wide enough to accommodate a non-structural 4-40 screw for harness routing if needed. The 

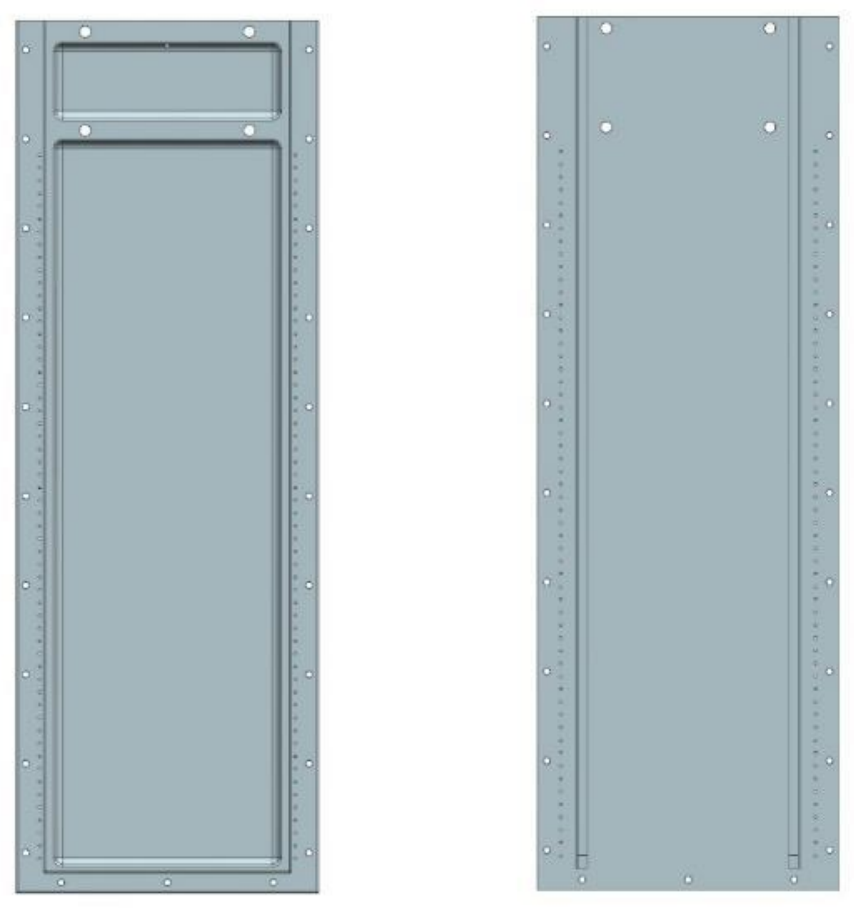

Figure 34 P-POD Mk. IV Top Panel

FEA was conducted, while hand calculations were used to determine the strength of the fastener through-holes. The finite element model used a symmetric constraint in order to reduce solving time, which solves a mirror image of the model across the centerline. All other edges were fixed. The Top Panel was subjected to the Y-axis load case, applied at the panel rails. The resulting Top Panel stress from FEA is shown below in Figure 35. Stress was very low throughout the entire part, the max being at the venting holes. This is expected as a hole in a panel will always produce stress concentration. Fortunately, the stress was already so low that even the max stress exhibited by the part yielded an M.S. of 4.6. The 3 4-40 through-holes at the $-Z$ end of the part exhibited a lower M.S. of 2.8, which is still very high. The Top Panel was not expected to be the limiting factor in considering P-POD strength, even after mass reduction. 


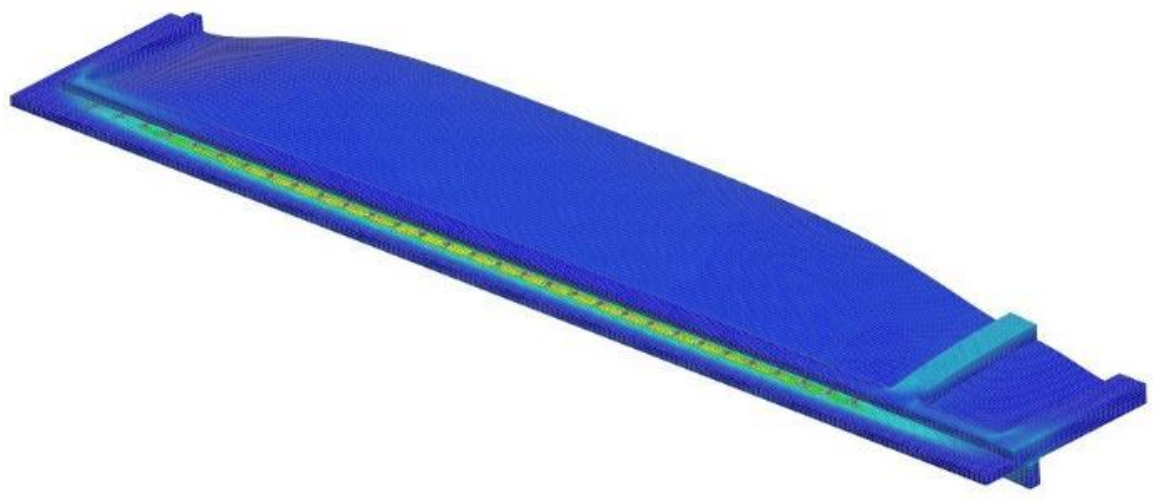

Figure 35 P-POD Mk. IV Top Panel FEA Results

P-POD Mk. IV Bottom Panel

The next part considered was the P-POD Bottom Panel. The Bottom Panel is similar to the Top Panel but lacks the bracket interface and venting holes. Addtionally, the Bottom Panel is commonly used as a mounting surface, and therefore needs to accommodate the mounting holes. The P-POD Mk. III Rev. E Bottom Panel is shown below in Figure 36. The original design features very thick ribs along the entire length of

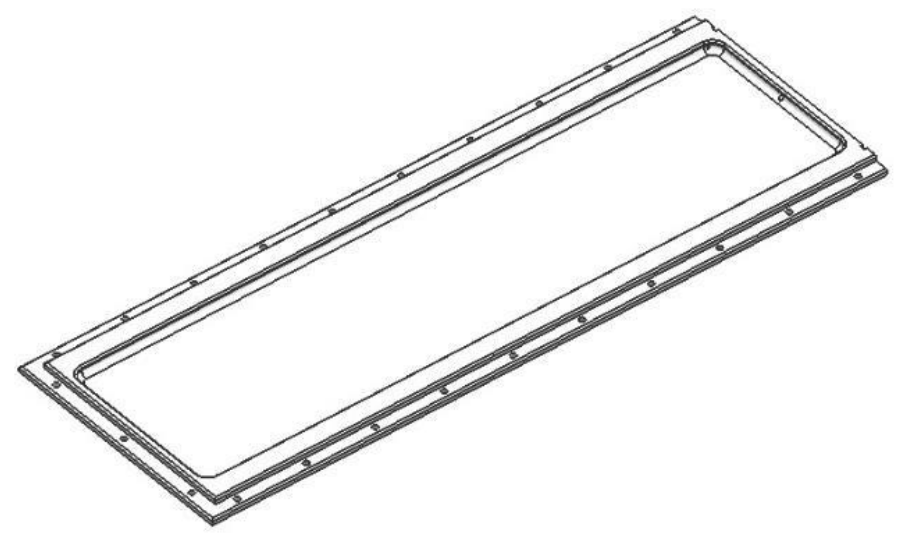

Figure 36

P-POD Mk. III Rev. E Bottom Panel

Page 53 
the part. These ribs are necessary at the mounting hole locations but excessive any where else. This was the primary method of reducing the mass of the Bottom Panel. Sections where the standard mounting pattern resides were left alone, and all other locatons had material removed. The resulting design is shown below in Figure 37. A thin rib was left on the exterior for retained stiffness, but material was cutout on both the inside and outside of the panel. One potential downside of this change is that it restricts the P-POD
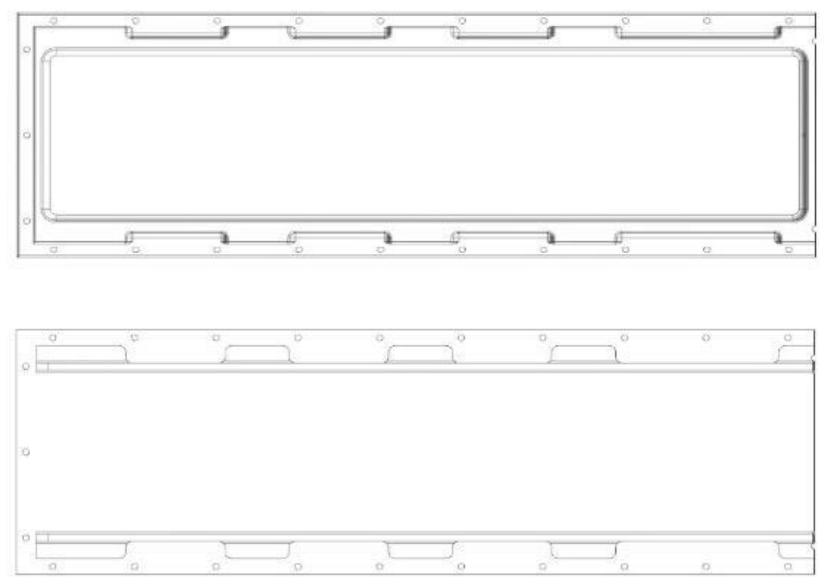

Figure 37 P-POD Mk. IV Bottom Panel

to utilize a single standard mounting pattern. This could prove to be a potential loss of flexibility down the road, but when considering the amount of launch vehicles that use any other mounting patterns than the 8-hole pattern proposed, this is acceptable. All launch vehicles that require different mounting patterns are either going out of production or can utilize old P-POD designs. An FEA was conducted to verify that the Bottom Panel can withstand CubeSat loads. The Bottom Panel FEM was subjected to a Y-axis load case applied to the rails. All interfaces to other parts of the P-POD were fixed. The 
resulting stress plot is show belown in Figure 38. The highest stress was exhibited near the fixed constraint, and was considered a somewhat unrealistic result. However, this value was well within the allowable range, still exhibiting a high positive margin, so the result was accepted as a worst case. Resulting Margin of Safety was 3.1 for the part stress. The area surrounding mounting hole locations was unchanged and not considered to be a stregnth risk.

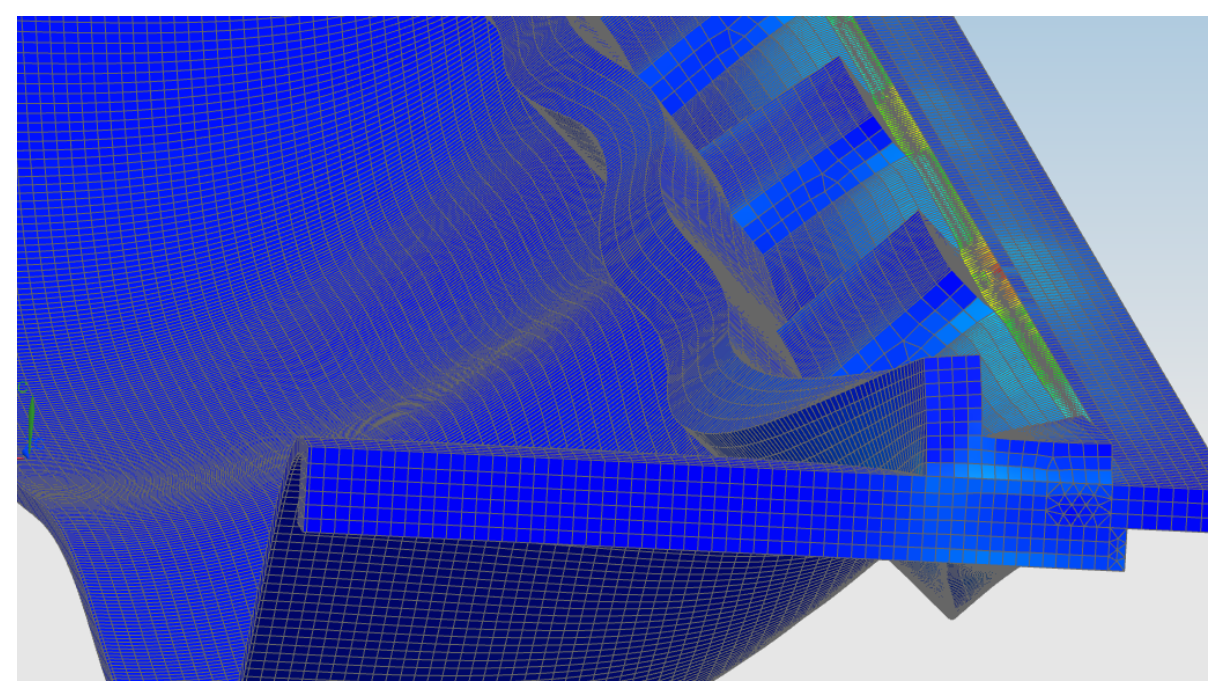

Figure $38 \quad$ P-POD Mk. IV Bottom Panel FEA Results

\section{P-POD Mk. IV Side Panel}

The next part considered was the P-POD Side Panel, that faces the $+/-\mathrm{X}$

direction. The P-POD Mk. III Rev. E Side Panel is shown below in Figure 39. The side panel contains access port windows that developers may utilize to access their satellite after it has been integrated into the P-POD. The location and size of the windows is specified in the CubeSat Design Specification (CDS) which is the standard document 


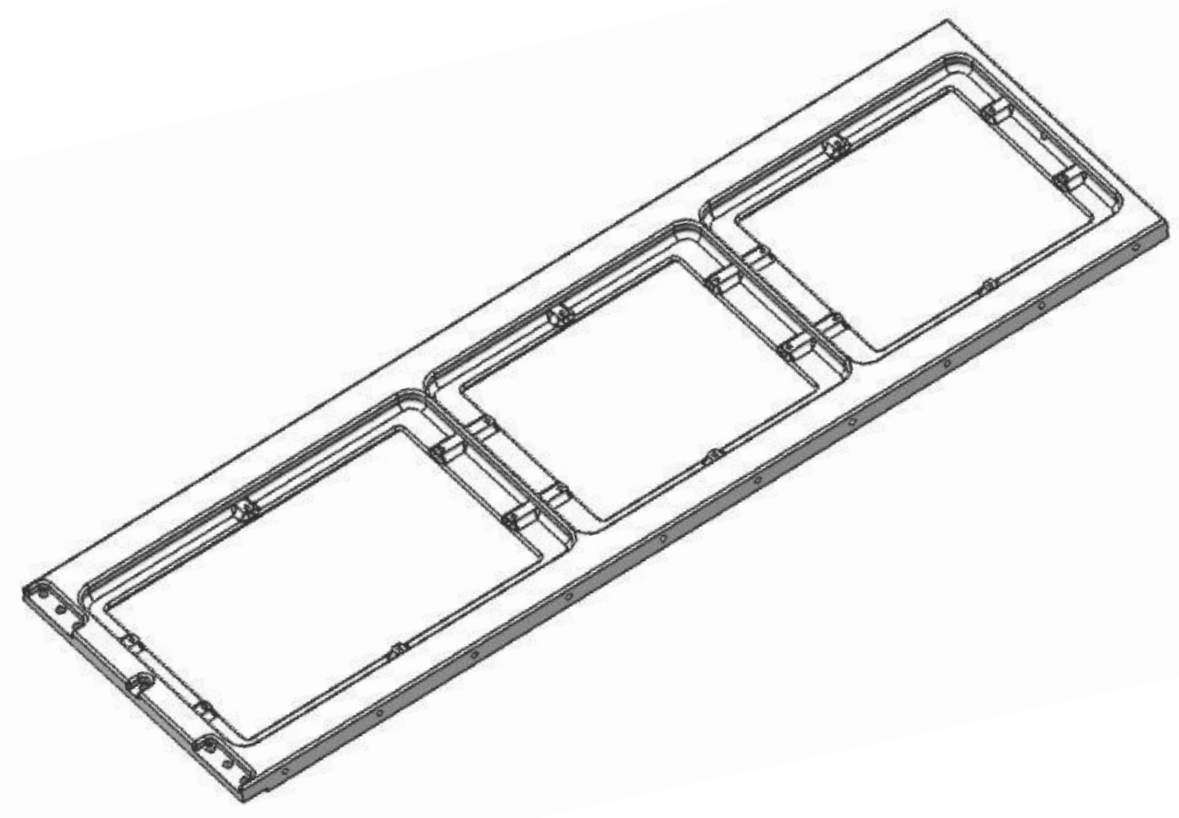

Figure 39 P-POD Mk. III Rev. E Side Panel

used to design and build CubeSats. Because these windows are in a standardized location, their size and position could not be changed. The P-POD EMI changes have however changed the access port cover interface. The access port covers now implement a flanged interface surface with an EMI gasket groove in place for maximum EMI signal attenuation. Additionally, because of previous experience with the size 2-56 screws used with the access port covers, the design was changed to utilize larger 4-40 torx head button cap screws, and 4 of them instead of 6 of the size 2 screws. This change makes the design more resiliant to vibrations as the size 2 screws back out easily. Other changes include removing material from areas that aren't utilized by the standard 8-hole mounting pattern in order to save mass. The resulting design is shown below in Figure 40. 

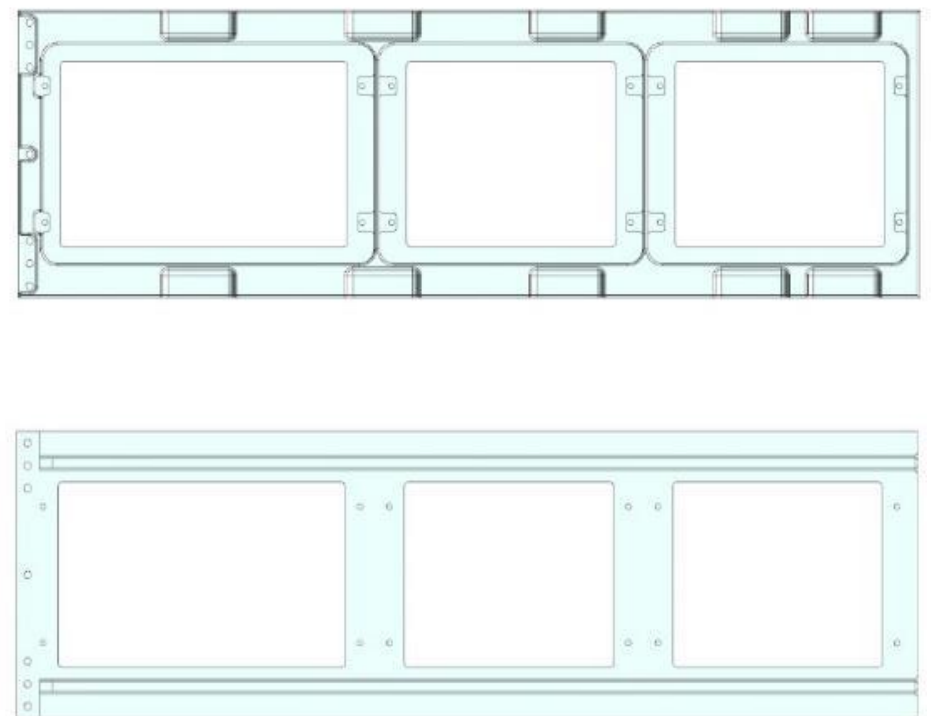

Figure $40 \quad$ P-POD Mk. IV Side Panel

Like the Bottom Panel, mounting flexibility is reduced, but in the long run, utilizing one mounting pattern can help reduce recurring engineering costs and provide a well known standardized mounting pattern. Aside from the mass reduction and access port mounting method, the side panel remains largely unchanged. An FEA was conducted to confirm the Side Panel's structural integrity, and it exhibited a high margin of safety of 6.4. A symmetric constraint was used to reduce solving time and all panel-topanel were assumed to be fixed. The X-axis load case described above was applied to the side panel rails. The resulting stress plot is shown below in Figure 41. The maximum stress was located near the fixed boundary constraint, and was well within acceptable levels. So far, all parts have exhibited extremely high margins. This is because of certain 
containment requirements, along with mounting interfaces that make the part more robust than it needs to be for the loads themselves.

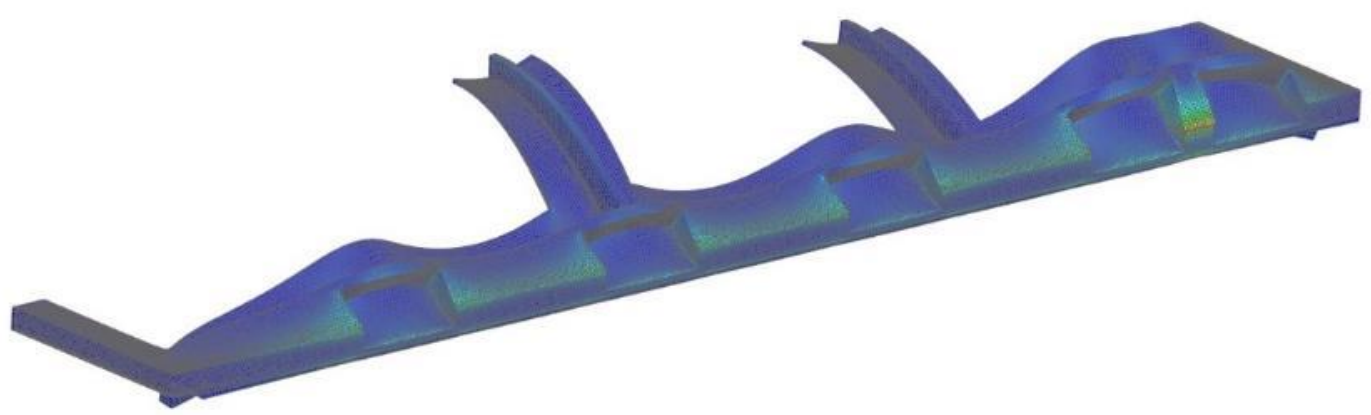

Figure 41 Side Panel FEA Results

P-POD Mk. IV Back Plate

The next part analyzed was the Back Plate, that makes up the $-\mathrm{Z}$ face of the $\mathrm{P}$ POD. In the past, this part was strengthened due to concerns with the screw interfaces having high stress concentrations, making the exterior walls thicker and taller, and adding more screws. The P-POD Mk. III Rev. E P-POD is shown below in Figure 42. These changes made the part very strong, but the thick, tall walls are only needed for areas where fasteners are located. In an effort to save mass, parts of the walls not near 


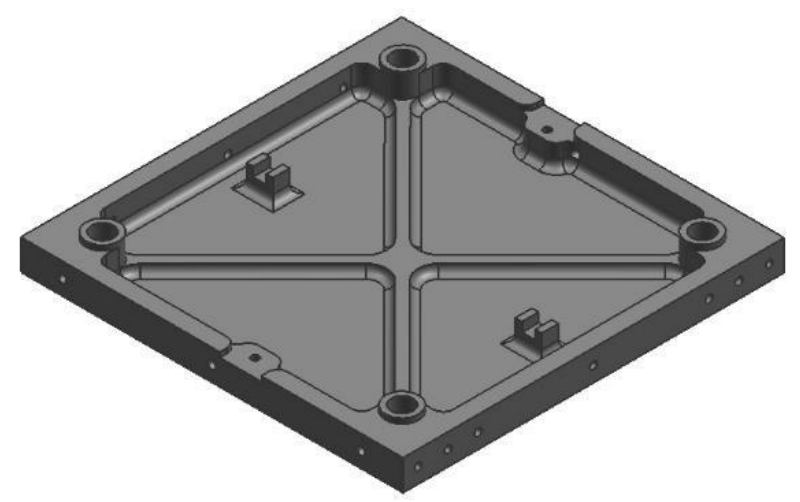

Figure 42 P-POD Mk. III Rev. E Back Plate

mounting screws or other features were thinned but remained constant height with the rest of the part. Additionally, four standoffs were added to streamline the implementation of a gaseous purge system or the Power-On system. If either system is required, the part can simply be sent out and have mission specfic holes drilled into it. The amount of material removed was low, saving only 12 grams, but it did not increase the part's complexity an appreciable amount. The Back Plate following the changes is shown below in Figure 43. Additionally, an example of the purge interface is shown in Figure 44. The part was expected to lose little to no strengh with this

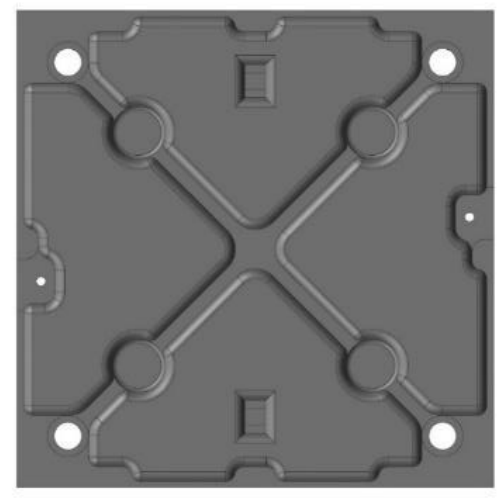

Figure 43 P-POD Mk. IV Back Plate Page 59 


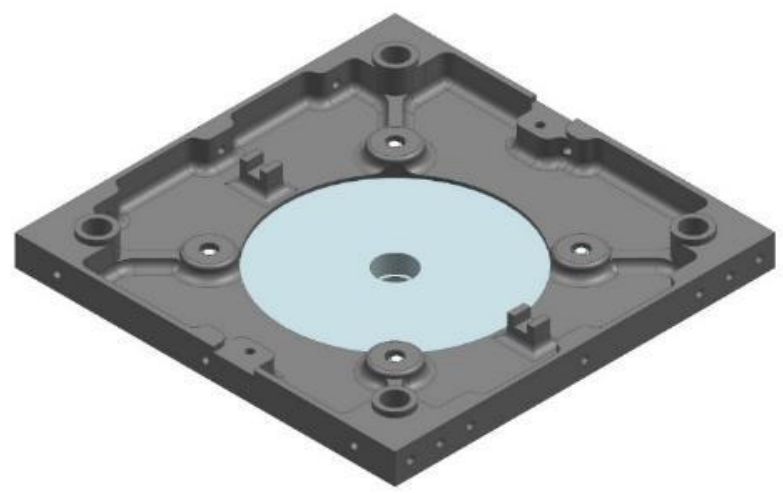

Figure 44 P-POD Mk. IV Back Plate Purge Interface

change, as no direct load path was altered and interfaces to other panels remained unchanged. In order to verify the prediction that the part retained its strength, an FEA was conducted under the Z-axis load case applied to the 4 spring plunger holes, which are the large holes shown in the corners of the figures above. All outer walls were considered fixed. The resulting stress is shown below in Figure 45. Slight stress was seen in each of the 4 corners, but this part exhibited and extremely high margin of safety of 10.5 . Another change to this part was the removal of the PTFE impregnated hard anodization, which required a high temperature bake to set. This bake would cause the material to have a strength equal to $80 \%$ of its expected yield strength. Because this component does not contact any CubeSat payloads, the low friction surface was not required, so in order to save time and cost, the PTFE impregnated hard anodization was removed. With the panel interfaces the same, the stress in the fastener holes was also the same, but because the part no longer has to go through the bake process, the margin of safety was even higher, going from 9.1 for the last version to 11.6 for the Mk. IV design. 


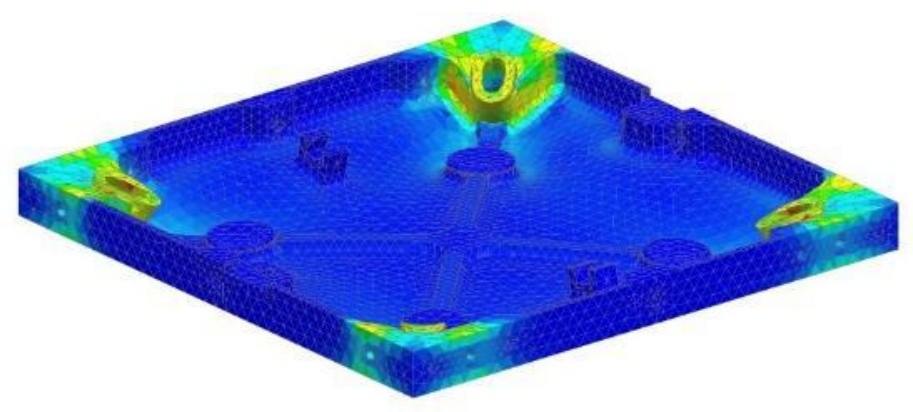

Figure 45 P-POD Mk. IV Back Plate FEA Results

P-POD Mk. IV Release Mechanism Bracket

The next part modified was the NEA Bracket that attaches to the +Y Top Panel. The purpose of the part is to mount the NEA so that it can constrain the door. Unlike the other parts, this part is not required to contain anything, so skeleton or truss structures are acceptable. The original Mk. III Rev. E Bracket is shown below in Figure 46. The objective was to shave some mass off of the part without dramatically decreasing its stiffness, and while maintaining a positive margin of safety. Another odd aspect of the bracket, was that the through holes used to attach the Bracket to the Top Panel were not centered on the part, and were located extremely close to one edge. This was most likely

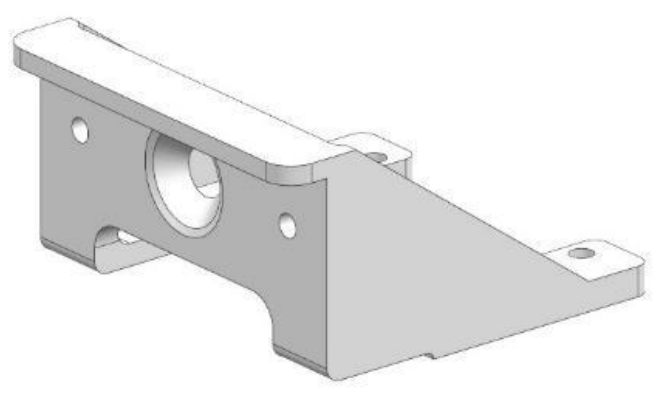

Figure 46 P-POD Mk. III Rev. E Bracket

Page 61 
a feature left over from a previous version of the P-POD which utilized shear pins in the bracket. The shear pins were removed back when the Mk. III Rev. C was released, but this feature was not changed. This design had heritage and did not pose a strength issue in the past, but moving forward there was no reason to maintain this odd design.

Material was removed from most sections of the part, reducing it to more of a skeleton like structure, as opposed to solid surfaces. Because the Bracket does not need to provide CubeSat containment, there is more liberty allowed in the structural design, unlike the rest of the parts on the P-POD. The resulting Bracket following the mass reduction changes is shown below in Figure 47. The side walls that bear the load from
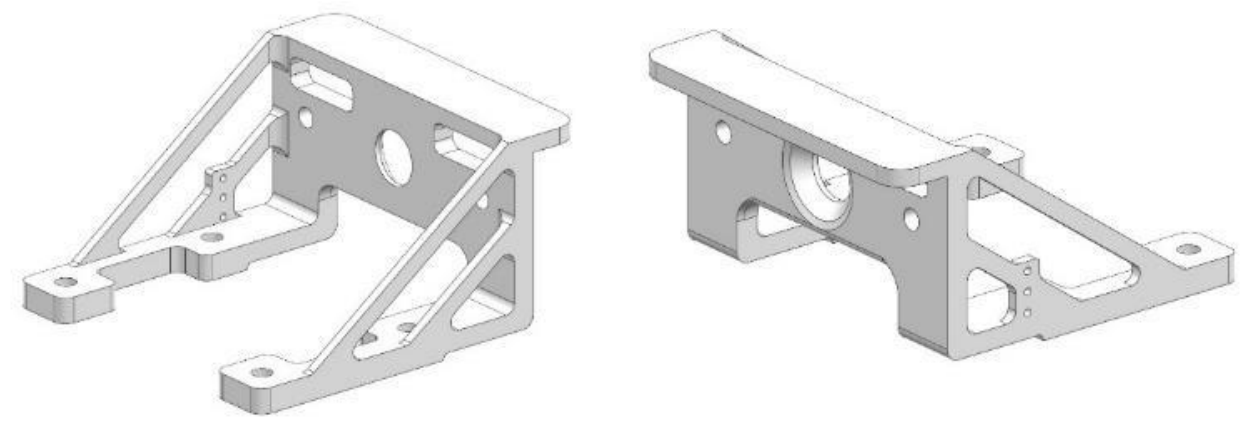

Figure 47 P-POD Mk. IV Bracket

the Door were reduced to two spars, and some material was removed in other sections that did not take any load, such as between the two mounting through holes, and the sections in the front face near the conical cup. Additionally, some material was removed from under the conical cup to accommodate the new Door geometry. Some of these changes reduced the front sections bending stiffness. In order to help rectify this, the lip that protrudes from the top edge of the bracket was heightened, to provide some extra 
stiffness. In order to evaluate the new design, an FEA was conducted. The part was fixed at its mounting holes. Beyond that, the model setup was a little more complicated than previous analysis thus far. The load imparted on the Door is transferred in unequal parts to the Bracket and the hinges on the Collar. Because of the geometry, the bracket takes $58 \%$ of the Z-axis loading. In reality, this load is transferred through the NEA Release Mechanism, as the door is attached to it through the Bracket. Therefore, to apply the load in the FEA, the load was applied to the NEA's section area that interfaces to the back face of the bracket, behind the conical cup interface. The FEA results are shown below in Figure 48. Areas of heighest stress include areas near the point where the top side spar meets the the back face, and under the part in the edge blend under where the

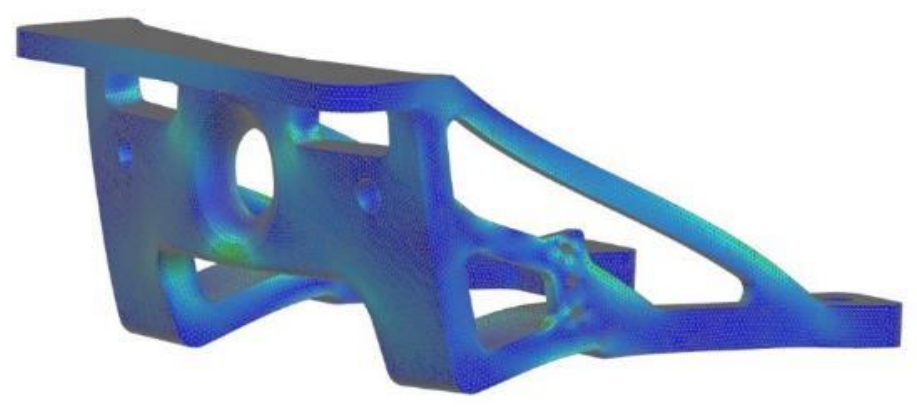

Figure 48 P-POD Mk. IV Bracket FEA Results

switch attaches to the bracket. These areas contain high concentrations of stress. Other areas of high load include the side spars themselves, and the area at the bottom of the conical cup, where material was removed to make room for the new door geometry, but these areas still exhibited high margins. The margin of safety at the high stress areas was 
0.8 , which is less then a third of that of the old design, but still well within the acceptable range. The Bracket changes resulted in a mass reduction of 25 grams.

P-POD Mk. IV Collar

The P-POD Collar saw no design changes, save for the addition of a gasket groove to accept the EMI gasket to seal the Door-Collar gap. This Collar is shown below in Figure 49. The only strength concern was that of the gasket groove causing stress concentrations. An FEA was conducted to check this prediction, with the Collar fixed at

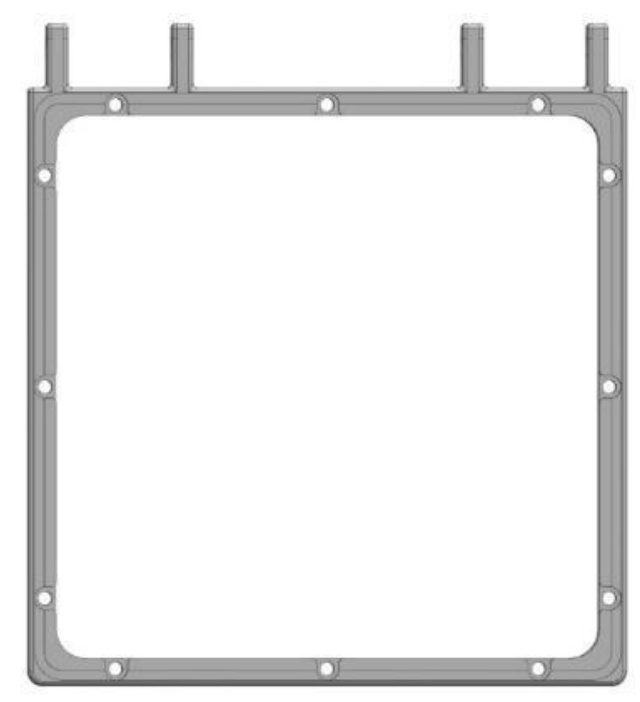

Figure 49 P-POD Mk. IV Collar with Gasket Groove

its mounting holes, with the Z-axis load left over from the Bracket analysis applied to the hinge hole. The results involved a slight increase in stress from the previous design, yielding a Margin of Safety of 0.4, down from 0.5 for the previous design. This decrease was not seen as an issue. The FEA results are shown below in Figure 50. 


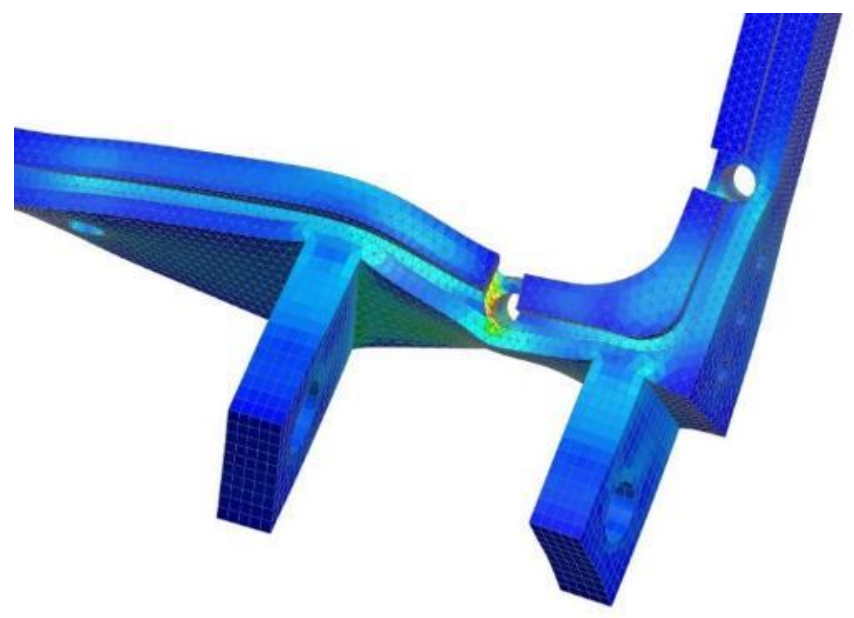

Figure 50 P-POD Mk. IV Collar FEA Results

P-POD Mk. IV Door

The next part evaluated was the Door. This part was seen as a part that was due for a redesign. It is consistently the weak point of the P-POD, and often deflects enough to exhibit a noticeable increase in door-collar gap. A stiffer door that could better compress the EMI gasket was desired. The P-POD Mk. III Rev. E Door is shown below in Figure 51. The first item addressed was the material coating employed on the P-POD Door. A PTFE impregnated hard anodization was used on the part, which requires a high temperature bake that lowers the yield stress to $80 \%$ of its original value. Because this part is the limiting factor of the P-POD, the need of this coating was seriously questioned. The next best alternative is simply using a standard hard anodization process, increasing the hardness of the running surface, while only taking a slight reduction in sliding friction. The sliding friction itself is only of minor concern, as the sliding contact the CubeSats have with the door is limited to the milliseconds following deployment. After 
which the door has had enough time to move out of the way of the CubeSat. This coating change itself causes a significant increase in margin of safety.

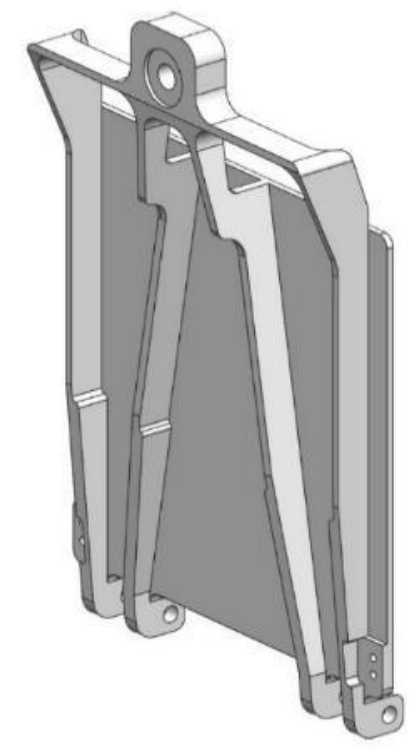

Figure $51 \quad$ P-POD Mk. III Rev. E Door

Hinge design modifications were not highly sought after due to how the current design maximizes the Door's movement away from the CubeSats. This is unfortunate as it imposed a limit on how much the part's margin could be increased. In the mean time, focus was shifted towards stiffening the part. The puzzling aspect of the Mk. III Rev. E Door, is that the stiff structure section is not directly on the load path between the CubeSat rail interface and the Door anchoring points. The more ideal case would be to have the CubeSat rails directly interface with the stiff structure of the door. A beam style design was used, using the geometry to maximize stiffness without a substantial increase in mass. From there, structure was routed towards the NEA anchoring point. The resulting Door design is shown below in Figure 52. The thicker ribs resulted in a mass 


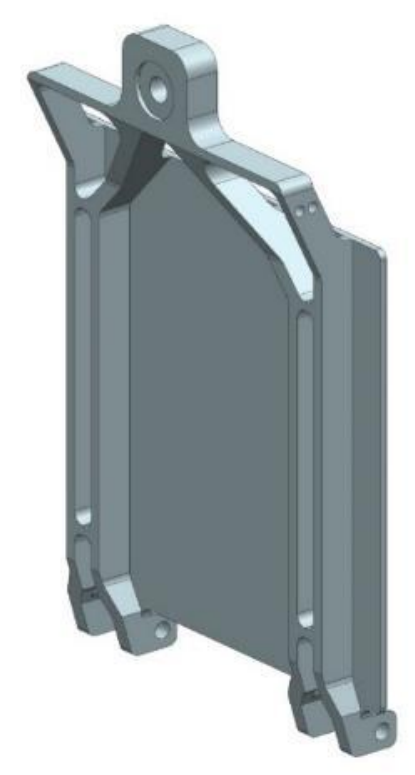

Figure $52 \quad$ P-POD Mk. IV Door Design

increase of 18 grams, but this was acceptable provided that the part was stiffer and stronger. In order to evaluate this part, an FEA was conducted. The hinge hole was constrained as a pin constraint to allow the door hinge to rotate around its axis. The release bolt hole and cone were fixed. The Z-axis load was applied to 4 square areas on the Door to simulate the manner the CubeSat loads the Door. Door deflection was evaluated to make sure no significant gaps were formed as a result of the loading that may lead to EMI leakage. Door deflection values for the P-POD Mk. IV Door were roughly one half those of the previous design, which was considered to be a success. The stress of the part exhibited a slight decrease in stress at the high stress point of the door, on the inner edge of the inner hinge. Coupled with the allowable stress inscrease due to the removal of the PTFE baking process for the coating, this part exhibits a significantly higher margin of safety than the previous design. The resulting analysis stress plots are shown below in Figure 53. Additionally, a hinge closeup is shown in Figure 54. The 


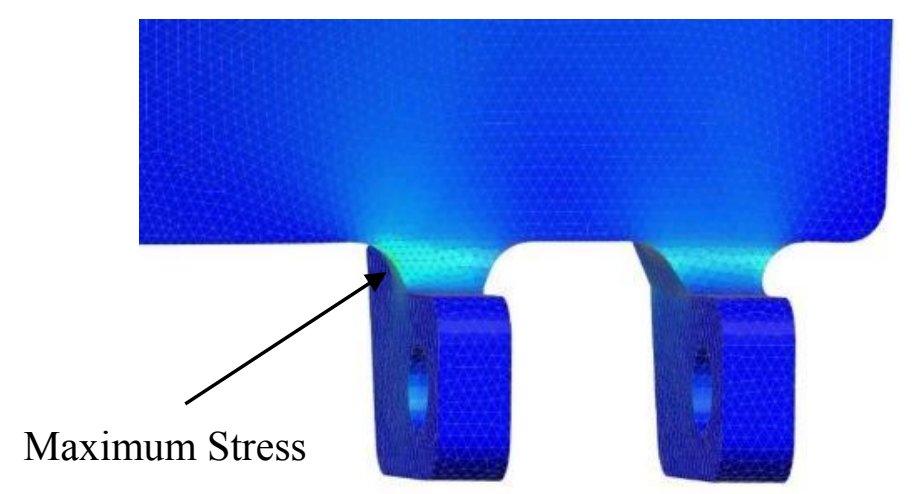

Figure $53 \quad$ P-POD Mk. IV Door Stress

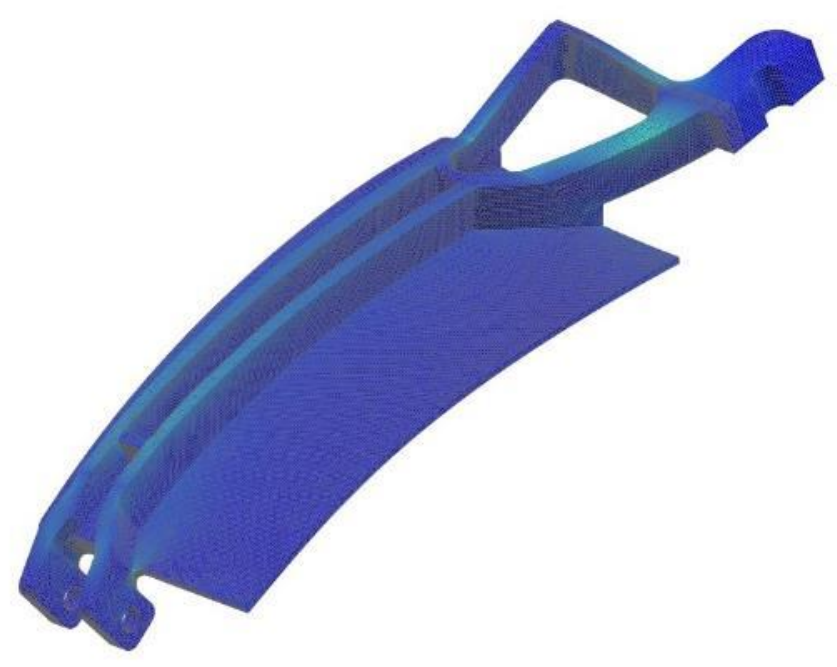

Figure $54 \quad$ P-POD Mk. IV Door Hinge Stress

hinge exhibits significant stress, but shows up light blue instead of red because of some severe stress elements near boundary conditions. The Margin of Safety at the maximum stress point depicted was 0.06 . This is significantly lower than other components of the P-POD, but the previous design exhibited a negative margin, so this was considered a 
significant improvement. The changes to the door resulted in a 18 gram mass increase, which was worth the improvement.

\section{P-POD Mk. IV Access Port Covers}

The access port covers were also redesigned to accommodate the EMI Gasket, as well as the changes to the mounting method described in Chapter 2. Through incorporating the flanged interface with gasket groove, the access port covers gained significant stiffness, such that all six screws were not necessary. A recap of the basic access port cover design is shown below in Figure 55. The access port covers are not
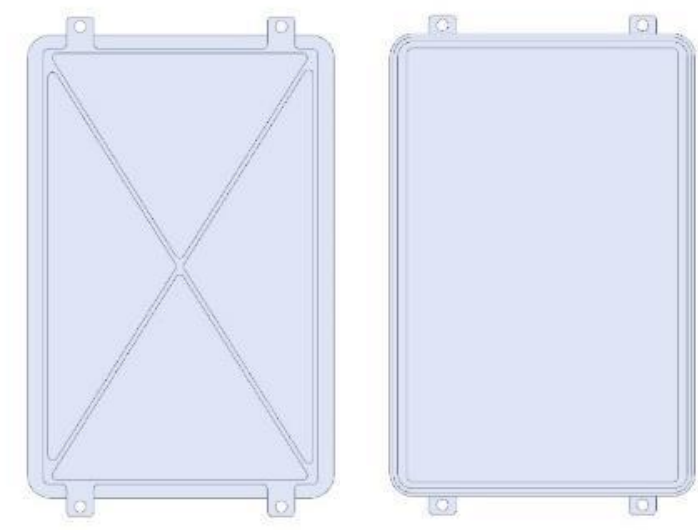

Figure 55 EMI Access Port Cover

intended to withstand any load from the CubeSat, and simply have to withstand the gravity load of their own weight. As expected, under the $\mathrm{X} / \mathrm{Y}$ gravity load factor the margin of safeties for each size were well over 10. It was important to also consider the load of part of the CubeSat resting on the access port during launch, should the CubeSat structure fail. In order to simulate part of the CubeSat resting against the access port cover, a load consisting of $1 \mathrm{~kg}$ at the $\mathrm{X}$-axis load factor was applied to the access port 
cover. Both the size access ports would survive the loading with a margin of safety of 0.1. The resulting stress plot from the FEA is shown below in Figure 56. Under this load case, the failure mode is the part yielding at the interior pocket corner and near the mounting holes. Additionally, an FEA was conducted using only the gravity load to ensure minimum deflection to confirm that no gaps would be created during launch that could allow EMI/RFI to escape. The large access port cover deflected more due to its size, but only 0.003 inches. The access port EMI gaskets are compressed 0.013 inches and would still be compressed with this deflection. The redesigned access ports offer no shortage of stiffness due to their flange design and gasket accommodation.

Unfortunately, in order to accommodate EMI gaskets, the access port cover set adds 134 grams compared to the previous access port cover set.

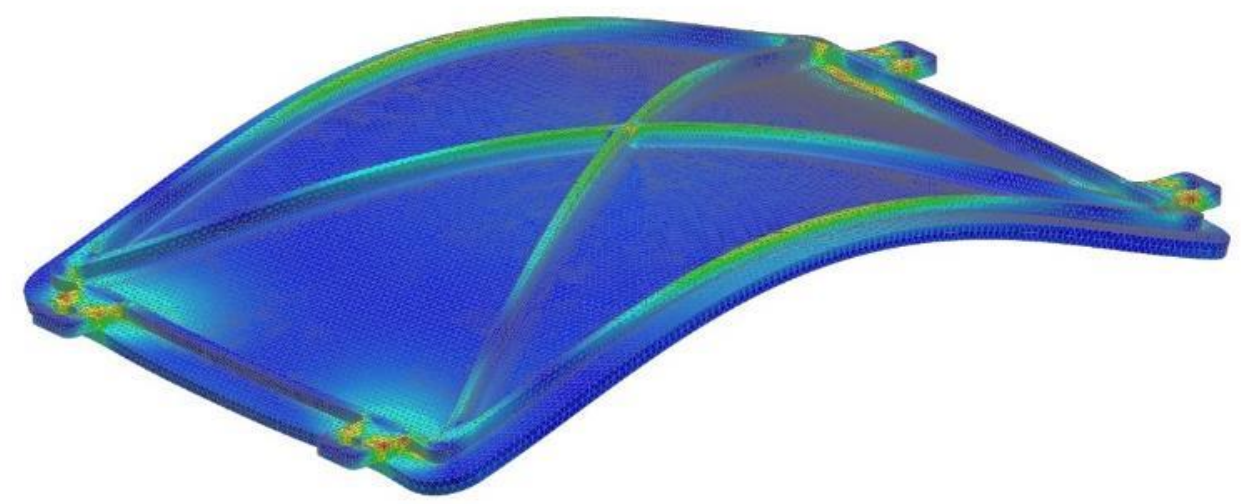

Figure 56 P-POD Mk. IV Large Access Port

P-POD Mk. IV Pusher Plate

The next component of interest was the pusher plate. The original design initiative of the new pusher plate was to provide additional volume for CubeSats. The Page 70 
volume within the P-POD mainspring was previously unused. Thus, the original "Tuna Can" Pusher Plate was designed for the P-POD Mk. III Rev. E. This design is shown below in Figure 57. This design added roughly 50 grams to the total mass of the P-POD, but provided additionaly volume for the CubeSat to use, and included a cylindrical wall that protects the CubeSat from any main spring movements. This pusher plate had the opportunity to prove its function on an Atlas V Launch, where a CubeSat had a large protrusion off of its $-Z$ face that fit inside the pusher plate. So the P-POD Mk. III Rev. E Pusher Plate's design intent was met, but it was unnecessarily bulky given the load cases it was seeing. Additionally, upon integration any spring misalignment would cause the spring to catch on the cylinderical section and then release moments later with an unattractive sound. This was undesireable and designed out for the P-POD Mk. IV Pusher Plate.
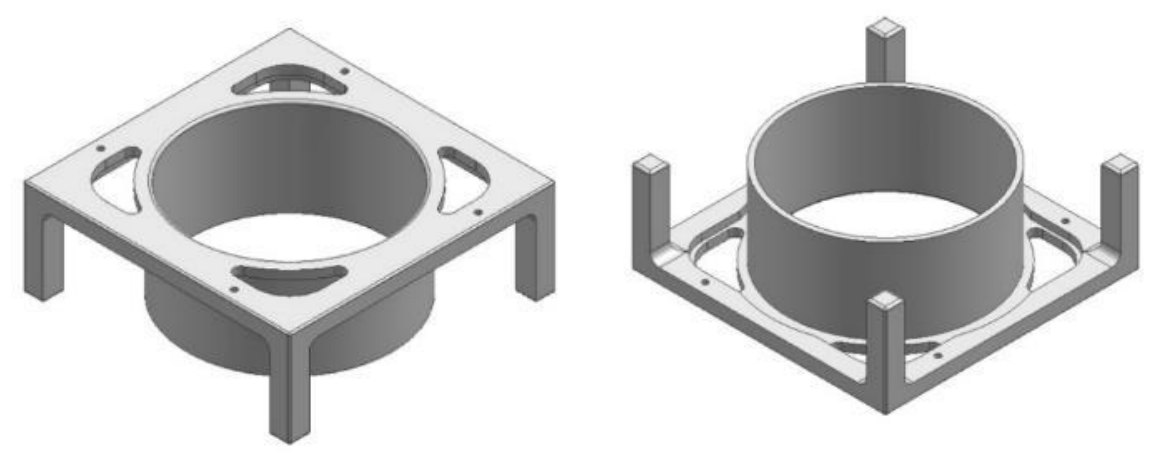

Figure 57 P-POD Mk. III Rev. E “Tuna Can” Pusher Plate

The new Pusher Plate for the P-POD Mk. IV sheds much of its mass without losing any of its utility. The only sections of the Pusher Plate that actually saw a significant load were its legs. These were left untouched, but the rest of the entire part 
was shaved down to be very slim, but because the part had a cylinder in the center of it, it still remained very stiff. Additionally, a chamfer was added to the outer edge of the slimmer tuna can wall which proved to completely eliminate the spring catching issue. The resulting re-design is shown below in Figure 58. This design saves 41 grams from the previous Pusher Plate design. The strength was verified by an FEA, subjecting the Pusher Plate to the Z-axis load acting downward on the legs. The margin of safety was slightly reduced from the previous design even though nothing on the load path of the Pusher Plate was changed. This was attributed to thinner structure at the point where the legs meet the rest of the part, causing a loss of stiffness in the corner. This margin of safety reduction from 11.5 to 11.1 was not seen as a serious problem as 11.1 is still very high.
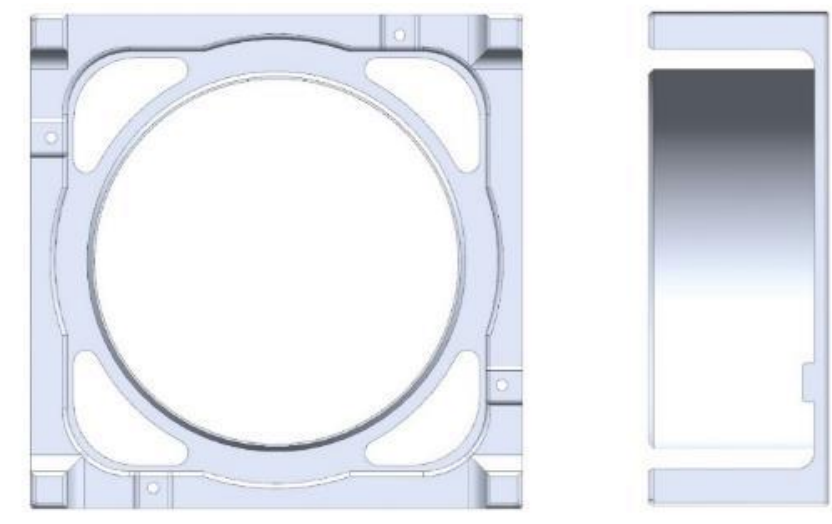

\section{Figure 58 P-POD Mk. IV “Tuna Can" Pusher Plate}

The majority of P-POD components exhibit extremely high margins. These high margins are a product of some of the geometric constraints that are levied on the P-POD design. The inner rails of the P-POD that are the running surfaces of the CubeSats are 
part of the CubeSat to P-POD interface and cannot be altered. These rails are a direct part of the load path and contribute a significant amount of strength to the structure. Additionally, the P-POD is required to contain the CubeSat in case of CubeSat failure. Therefore, the P-POD panels must include extra material to create a continuous enclosure. These factors, along with limiting machining complexity, contribute to the design of some components being left with excessive margins of safety.

\section{P-POD Structural Redesign Summary}

Following all design changes, the P-POD's overall margin of safety was increased while its mass was decreased, which can save cost and/or accommodate higher payload masses. EMI modifications added 134 grams to the P-POD mass, while the Mk. IV Door is also 18 grams heavier than the Mk. III Rev. E Door. Despite these increases, the total mass reduction for 1 P-POD was 154 grams. Additionally, the overall margin of safety for yielding under the analyzed load case was raised from -0.2 to 0.06 . This negative margin in the Mk. III Rev. E design is not surprising, as the Mk. III P-POD was tested to and survived the same levels that the design load case was derived from, but did exhibit signs of slight yielding of the door, in the form of increased door gaps. The important point, however, is that the P-POD is both lighter and stronger. Some points for further investigation include the prospect of completely re-designing the P-POD door hinge. Initially, removing the radius on the door hinge was not desired, because its geometry helps the door get out of the way of the CubeSat upon deployment. The radius hinge design also creates a stress concentration which is the cause of the low margin of safety. This deployment concern could be mitigated, which would allow for an even stronger door design to further expand the allowable CubeSat payload mass. An investigation and 
redesign of the hinge could improve the strength of the Door and bring it up to the strength of the rest of the P-POD. Additionally, looking at an alternative access port design without the flange and gasket groove could provide even further mass savings. For some missions that mount high quantities of deployers (8-16), 100 grams per P-POD is a substantial mass difference, and if EMI is not needed it is a waste of mass to incorporate the EMI access port covers, as there is really no structural reason for having such strong access port covers. A CAD assembly of the final P-POD Mk. IV design is shown below in Figure 59.

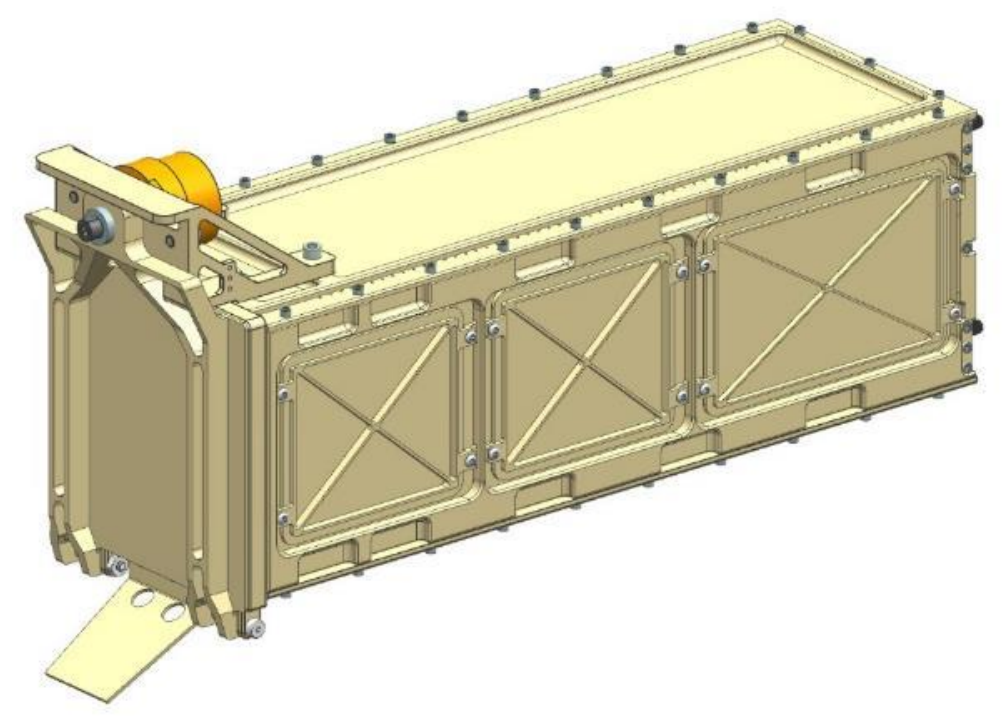

Figure 59 P-POD Mk. IV Final Design Assembly 


\section{CHAPTER V: ENVIRONMENTAL QUALIFICATION AND EMI TESTING}

P-PODs are subject to significant random vibration and thermal loads during launch. Everytime a new P-POD is going to fly on a launch vehicle, an engineering unit has to be put through a qualification program consisting of severe random vibration levels and extreme temperature cycling. Launch vehicle providers determine both the vibration and thermal maximum predicted environments (MPE) at the P-POD to LV interface. These environments are then used to derive qualification levels. In accordance with Launch Services Program Program Level Dispenser and CubeSat Requirements Document (LSP-REQ-317.01), qualification random vibration testing is conducted at $\mathrm{MPE}+6 \mathrm{~dB}$ for 3 minutes in each axis. In the same document, qualification thermal vacuum cycle specification is described as 8 cycles with 1 hour dwell times at levels equal to $\mathrm{MPE}+/-10^{\circ} \mathrm{C}$, at a vacuum level of $1 \times 10-4$ Torr in accordance with MIL-STD1540. The primary LV of interest for this program was the Atlas V, which accommoades the Naval Postgraduate School CubeSat Launcher (NPSCuL). The NPSCuL holds 8 PPODs and is mounted to the aft bulkhead of the Centaur stage of the Atlas V. The environments seen by the P-POD in this configuration are considered to be some of the most severe. Additionally, there is one short duration random vibration profile on the Falcon $9 \mathrm{LV}$, that is only specified for 17 seconds per axis, that is not encompassed by the P-POD to NPSCuL interface levels. In an effort to get the most out of this qualification unit, the decision was made to test to this profile following the qualification program on the NPSCuL to cover most future launch vehicles. The thermal levels on the Falcon 9 are much less severe and easily encompassed by the Atlas V/NPSCuL thermal levels. 


\section{Random Vibration Qualification Compliance}

The objective was to qualify the P-POD Mk. IV design for flight on both the Atlas $\mathrm{V}$ and the Falcon 9 v1.1 launch vehicles. Random vibration qualification levels are different for each, so the P-POD Mk. IV Qualification Test Program must encompass both vehicle profiles.

\section{Atlas V Random Vibration Qualification}

The random vibration levels for the NPSCuL can be found in the Atlas V Aft Bulkhead Carrier (ABC) User Guide (ABC_User_Guide_2014.pdf), which specifies the MPE random vibration profile described below in Figure 60 and Table 13.

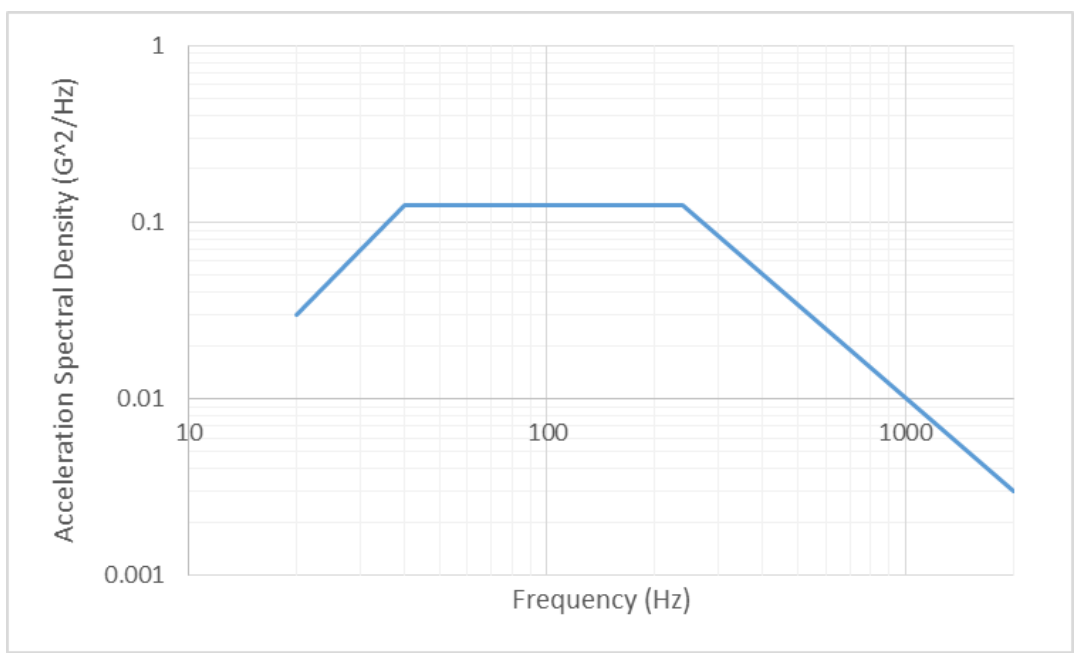

Figure 60 NPSCul to Aft Bulkhead Carrier Levels

Table 13 NPSCuL to Aft Bulkhead

Carrier Levels

\begin{tabular}{|c|c|}
\hline Frequency $(\mathrm{Hz})$ & ASD $\left(\mathrm{G}^{2} / \mathrm{Hz}\right)$ \\
\hline \hline 20 & 0.03 \\
\hline 40 & 0.125 \\
\hline 240 & 0.125 \\
\hline 2000 & 0.003 \\
\hline Overal & $7.6 \mathrm{G}_{\mathrm{rms}}$ \\
\hline
\end{tabular}

Page 76 
It is important to note that the levels described above differ then the levels designed to because these levels are the levels that the NPSCuL sees at its interface to the aft bulkhead of the Centaur stage, not the levels that the P-POD sees. The NPSCuL responds to those levels and produces an entirely different set of levels that act on the PPOD. The P-POD was mounted in the position on the NPSCuL that provides the worst case load scenario, and the same position that was used to derive the Mk. IV design load. Those levels have been characterized before in a previous test and are desribed at the beginning of Chapter IV. Adding $6 \mathrm{~dB}$ to the levels described above yields the qualification levels that the NPSCuL was tested to. These levels are described below in Figure 61 and Table 14.

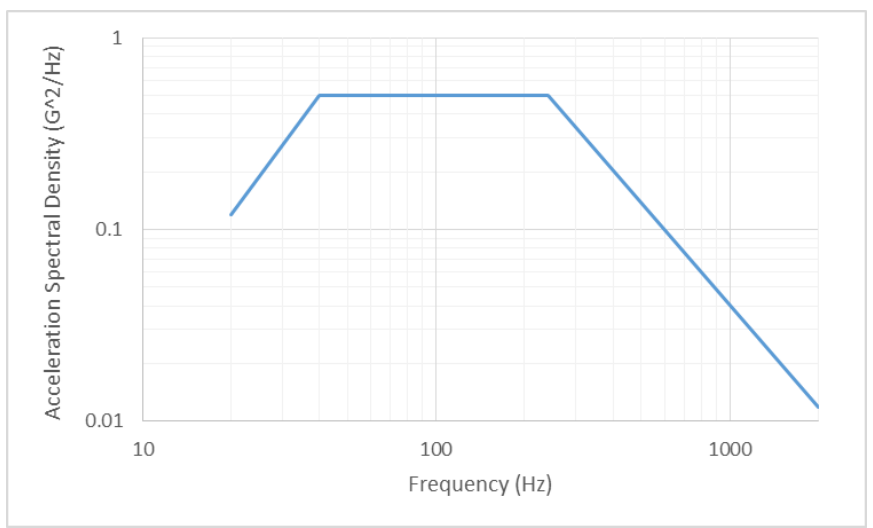

Figure 61 NPSCuL to Aft Bulkhead Carrier Qualification Levels

Table 14 NPSCuL to Aft Bulkhead Carrier Qualification Levels

\begin{tabular}{|c|c|}
\hline Frequency $(\mathrm{Hz})$ & $\mathrm{ASD}\left(\mathrm{G}^{2} / \mathrm{Hz}\right)$ \\
\hline \hline 20 & 0.120 \\
\hline 40 & 0.500 \\
\hline 240 & 0.500 \\
\hline 2000 & 0.012 \\
\hline Overal & $15.24 \mathrm{G}_{\mathrm{rms}}$ \\
\hline
\end{tabular}

Page 77 
The P-POD mounted to the NPSCuL in testing configuration is also shown below in Figure 62.

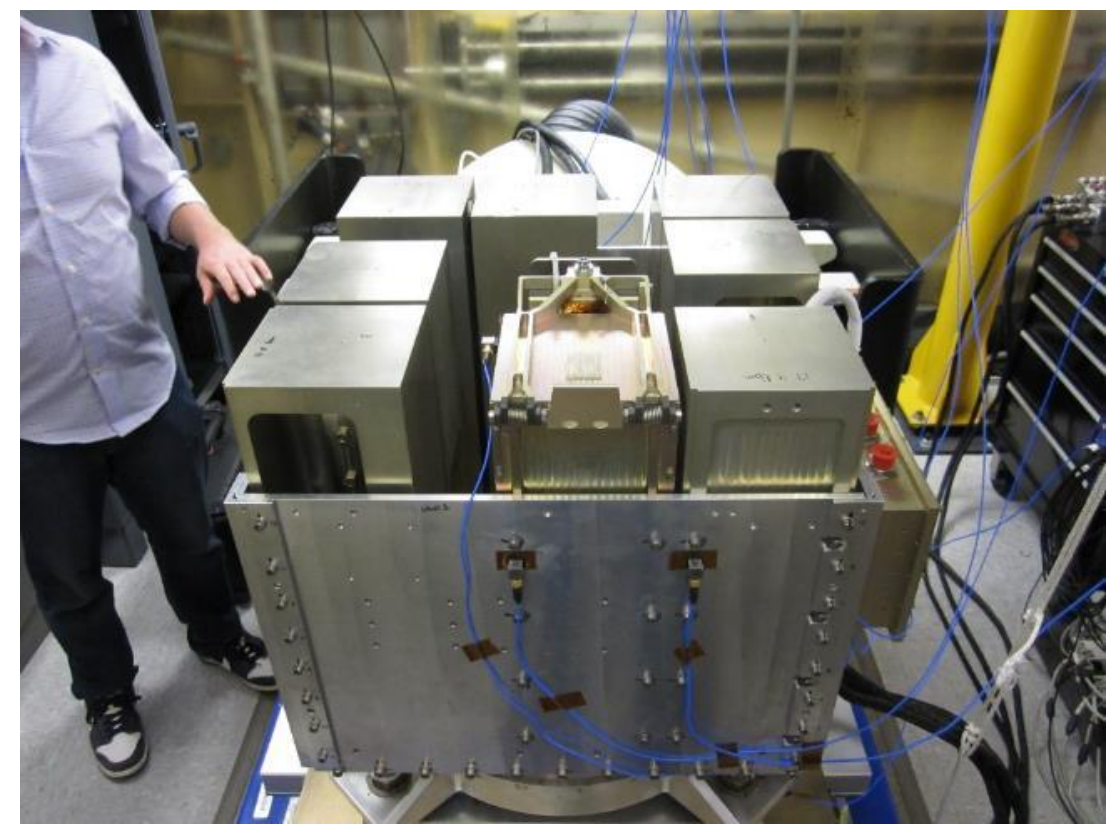

Figure 62 P-POD Mounted to NPSCuL in Testing Configuration

The NPSCuL with the qualification unit P-POD was tested to the above levels for a duration of 3 minutes in each axis. No anomalies were found when inspecting the PPOD, and P-POD interface response data for Z-, Y-, and X- axis tests are shown in Figure 63, Figure 64, and Figure 65, respectively.

On the control curve of the plots, some notches are seen for each axis. These notches are caused by the force-limiting system utilized by the NPS Random Vibration testing setup. The force-limiting system works by setting the maximum expected force to be seen by the shaker and ensuring this force is not exceeded due to the natural frequency of the test 
article. This is accomplished by mounting the NPSCuL interface ring to the interface plate through force transducers, and using these force meters a closed loop feedback for the drive input. P-POD deployment switch measurements remained nominal for the duration of the test with no instances of the switch opening, and post test inspection showed the P-POD was still in functional order following the random vibration qualification test. At this point a comprehensive inspection was done in accordance with the P-POD Acceptance Checklist (PAC).

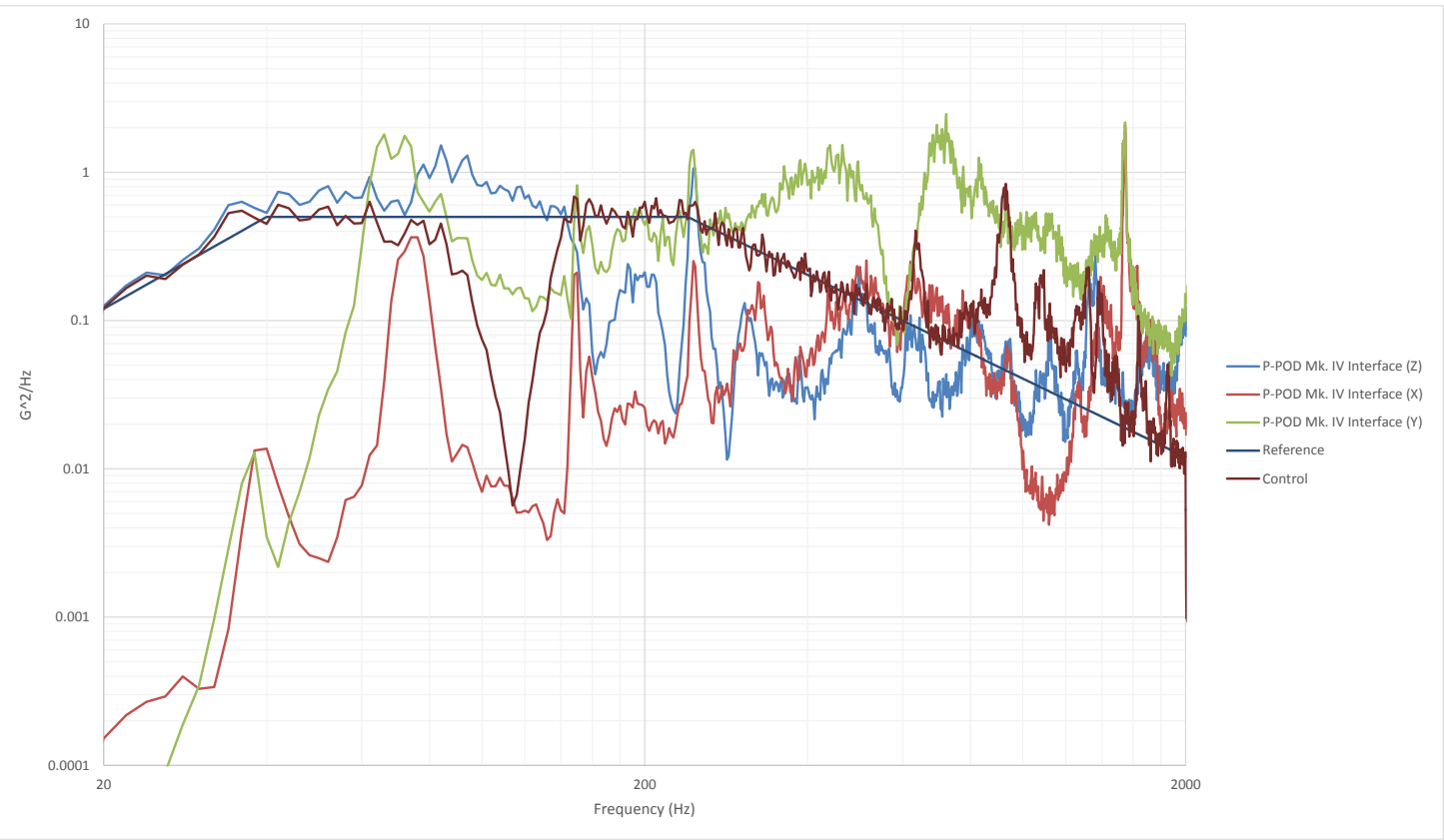

Figure 63 


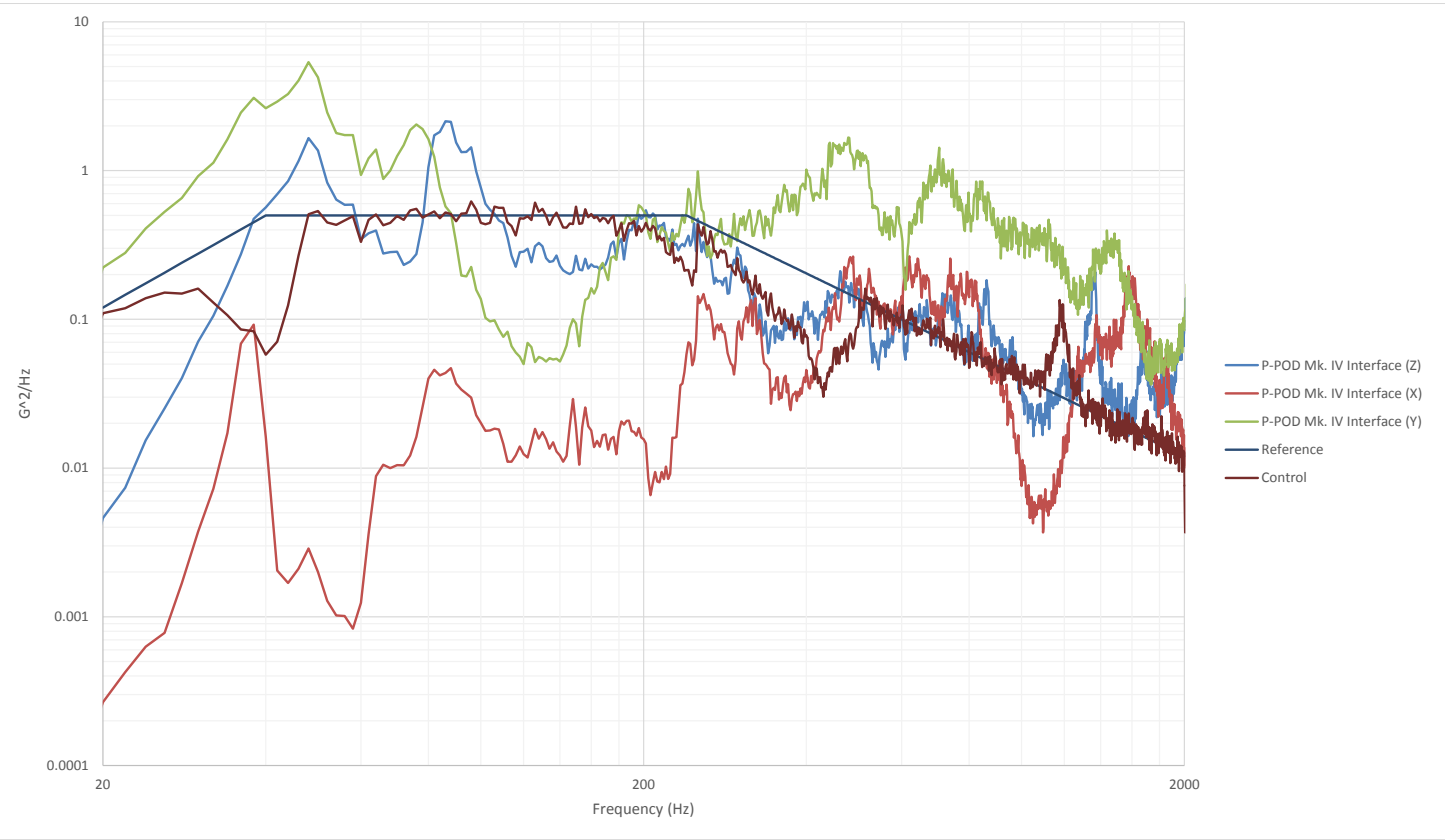

Figure 64 P-POD Y-Axis Vibration Test Data

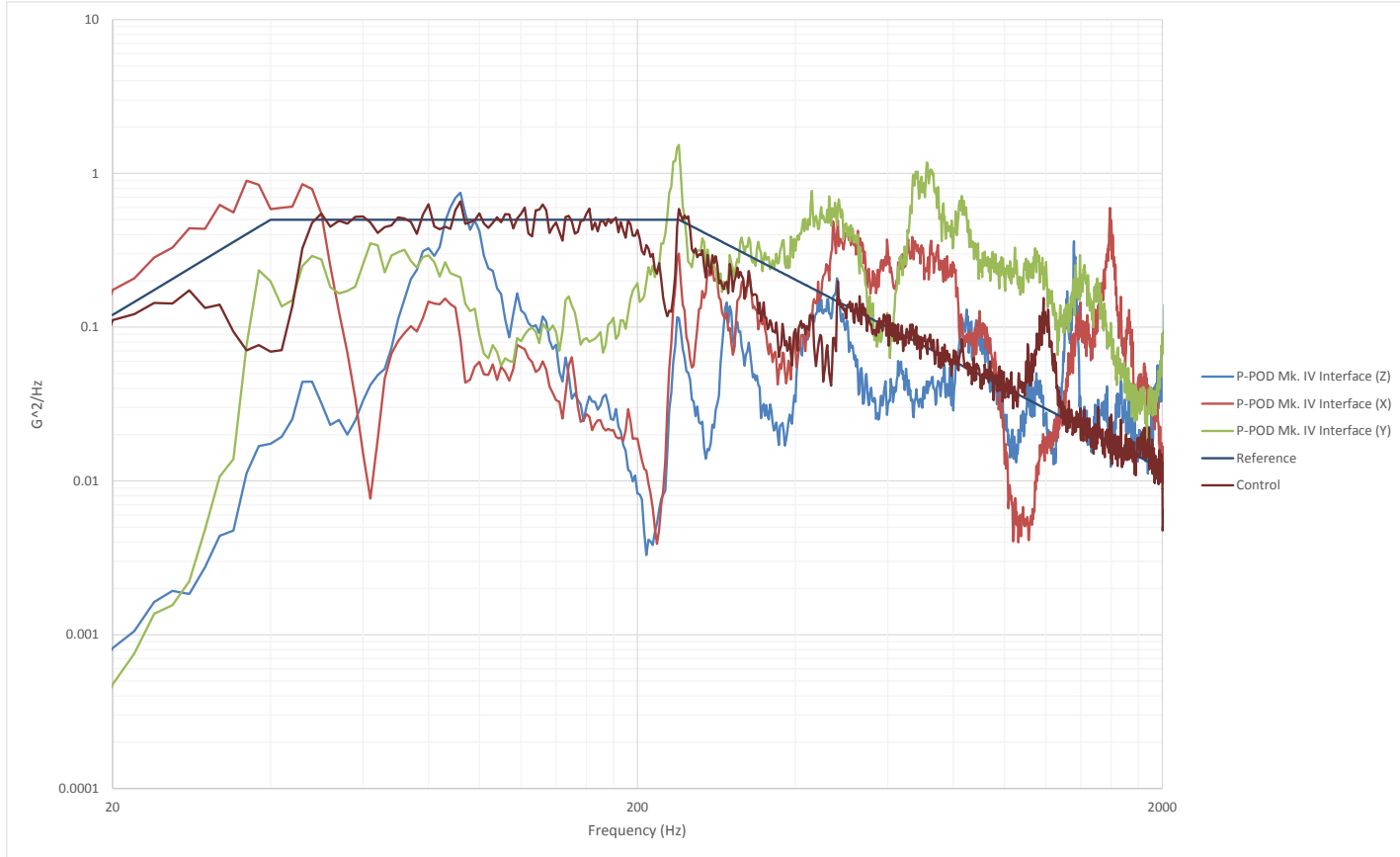

Figure 65 P-POD X-Axis Vibration Test Data

Page 80 
No damage to the P-POD's structure was observed during post test inspection. No fasteners dialed out and most fastener staking remained intact. In the case of 3 size 440 screws that affix the Bottom Plate to the Back Plate, a small section of the staking appeared to be delaminated from the fastener head. The majority of the staking still attached to the fastener showed that the fastener had not dialed out, but to ensure this remained the case during the next phase of random vibration testing, the three fasteners in question were torque-striped to easily visualize any evidence of fastener loosening.

\section{Falcon 9 Random Vibration Qualification}

The next random vibration qualification test was done to the Falcon 9 short duration levels. Because we do not need an NPSCuL to test to these levels, this test was done in house in the Cal Poly aerospace structures lab. The P-POD was mounted on its Y Bottom Panel to a magnesium interface block. This block is attached to the slip table to do X-and Z- axis testing, and mounted directly to the shaker in veritical configuration to conduct testing in the Y-axis. These levels are very severe in the low frequency range, but only require testing time for 17 seconds in each axis. The levels are described below in Figure 66 and Table 15. 


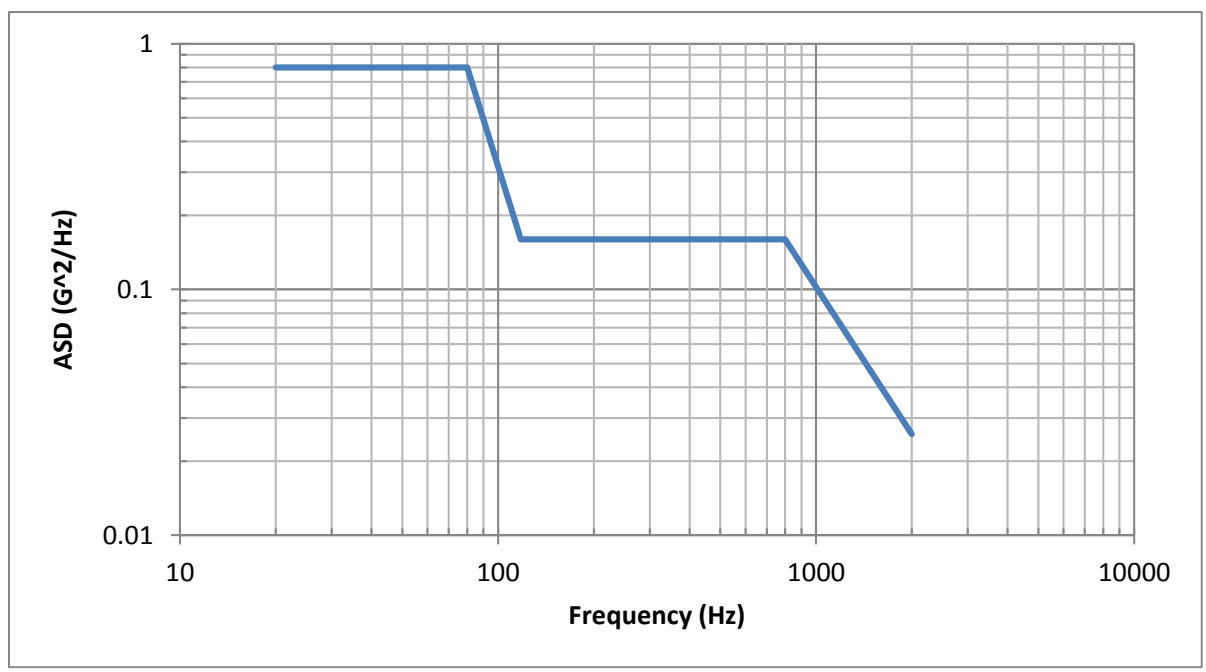

Figure 66 Falcon 9 Short Duration Qualification Levels

Table 15 Falcon 9 Short Duration Qualification Levels

\begin{tabular}{|c|c|}
\hline Frequency $(\mathrm{Hz})$ & ASD $\left(\mathrm{G}^{2} / \mathrm{Hz}\right)$ \\
\hline \hline 20 & 0.80 \\
\hline 80 & 0.16 \\
\hline 118 & 0.16 \\
\hline 800 & 0.16 \\
\hline 2000 & 0.0258 \\
\hline Overal & $15.76 \mathrm{G}_{\mathrm{rms}}$ \\
\hline
\end{tabular}

The P-POD mounted to the shake table in testing configuration is also shown below in Figure 67. 


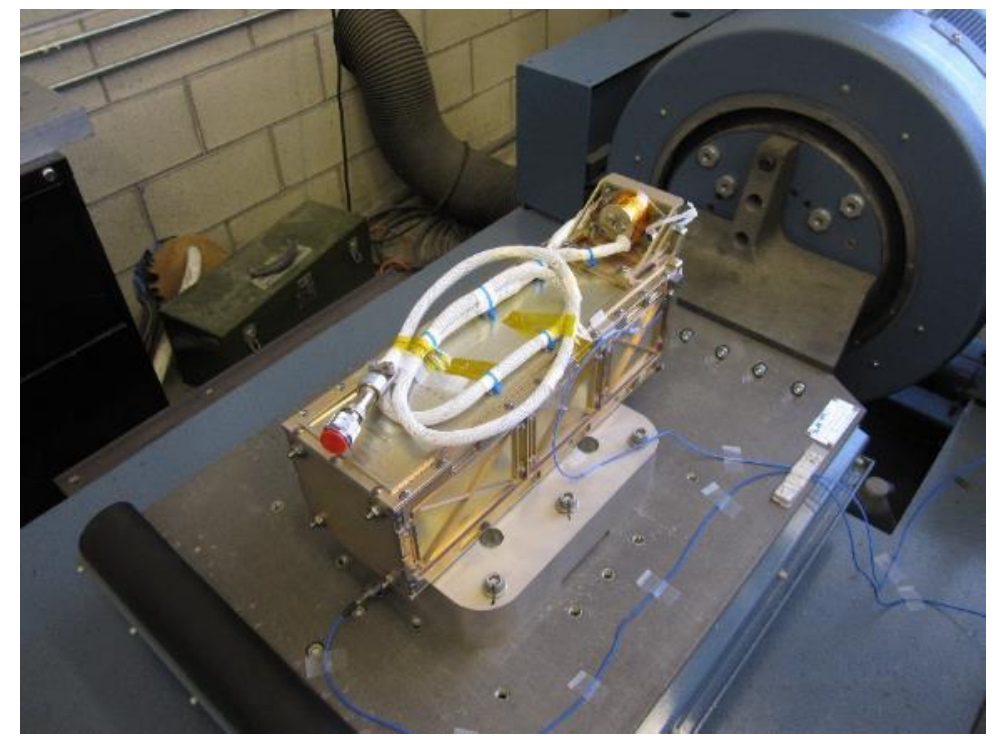

Figure 67 P-POD Mounting Configuration for CRS-3 Qualification

Following random vibration testing, no anomalies were found when inspecting the P-POD, and P-POD response data for $\mathrm{X}-, \mathrm{Y}-$, and $\mathrm{Z}$ - axis tests are shown in Figure 68, Figure 69, and Figure 70, respectively.

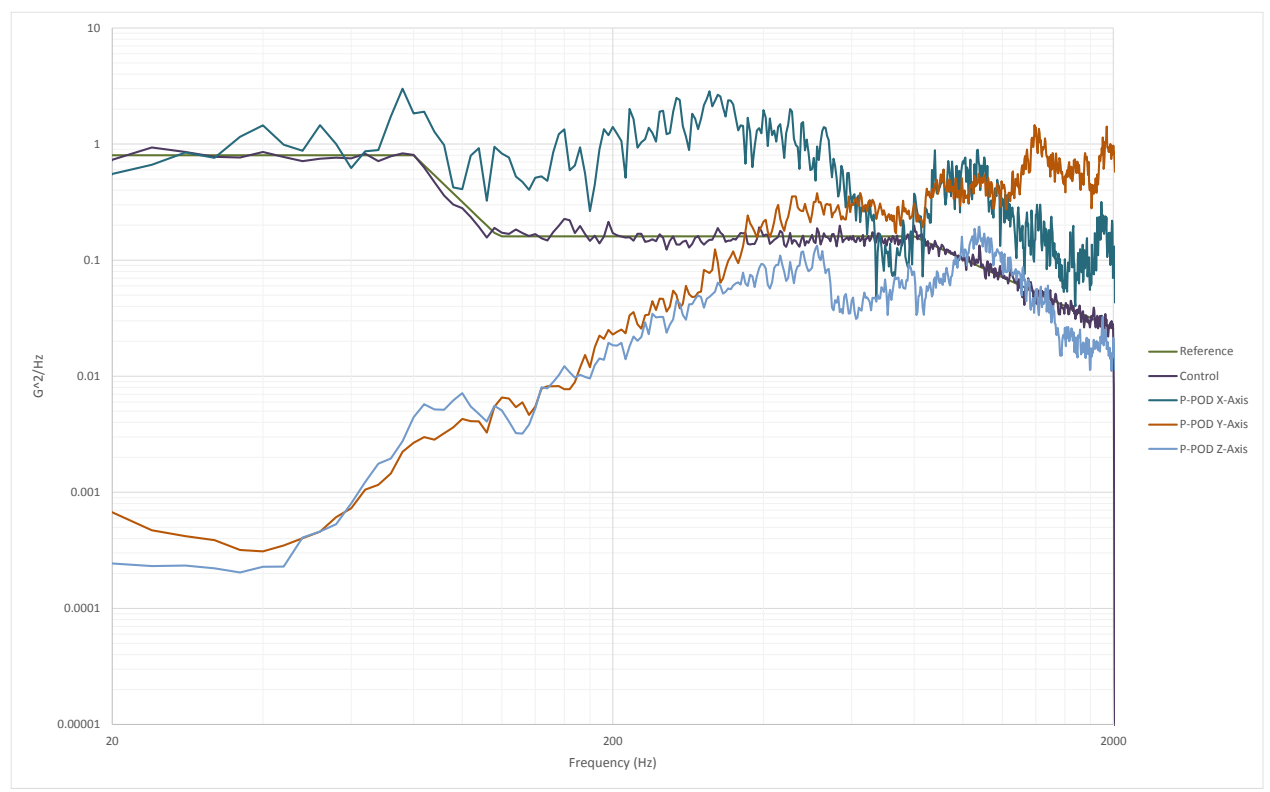

Figure 68 CRS Short Duration X-Axis P-POD Response Data Page 83 


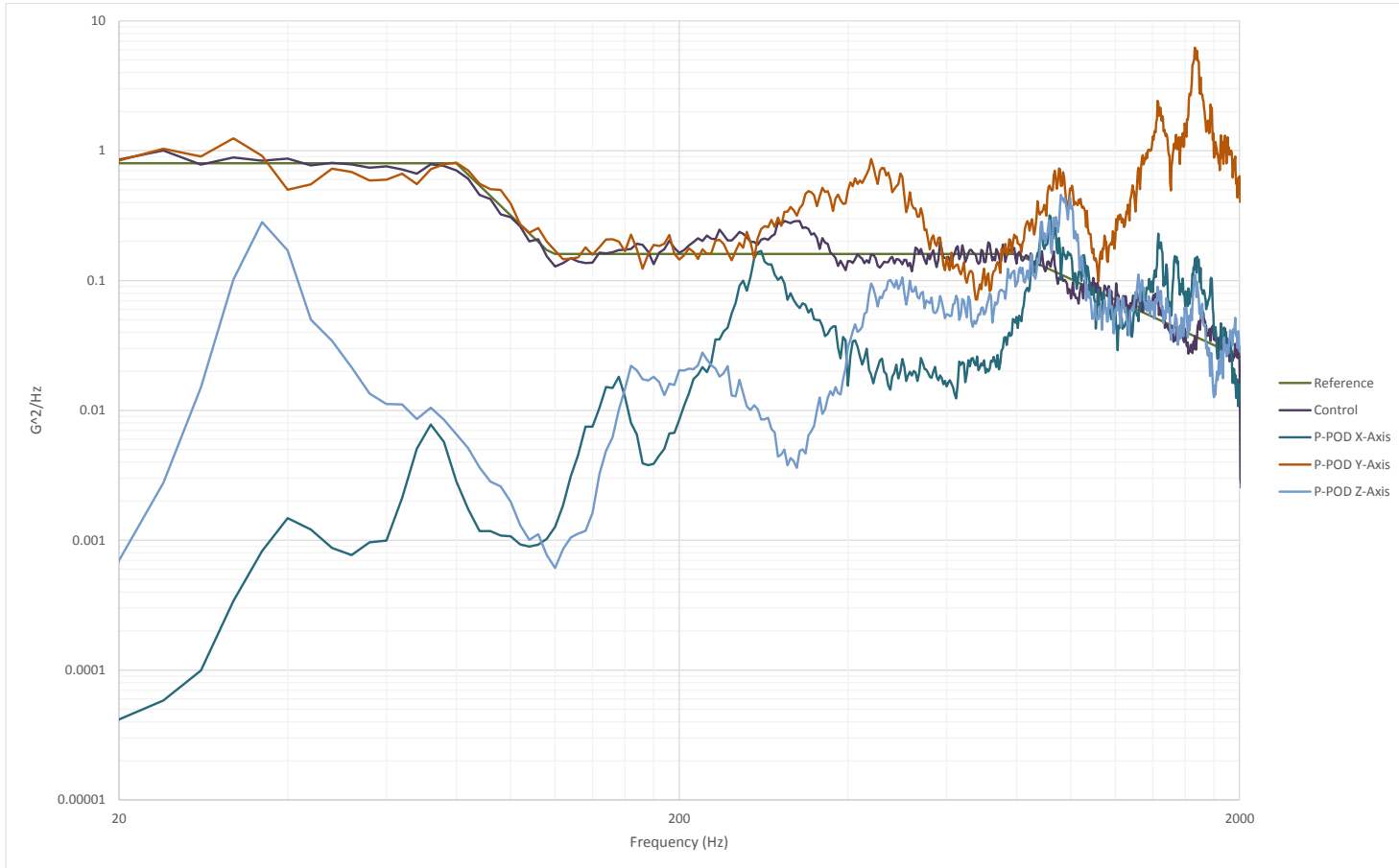

Figure 69 CRS Short Duration Y-Axis P-POD Response Data.

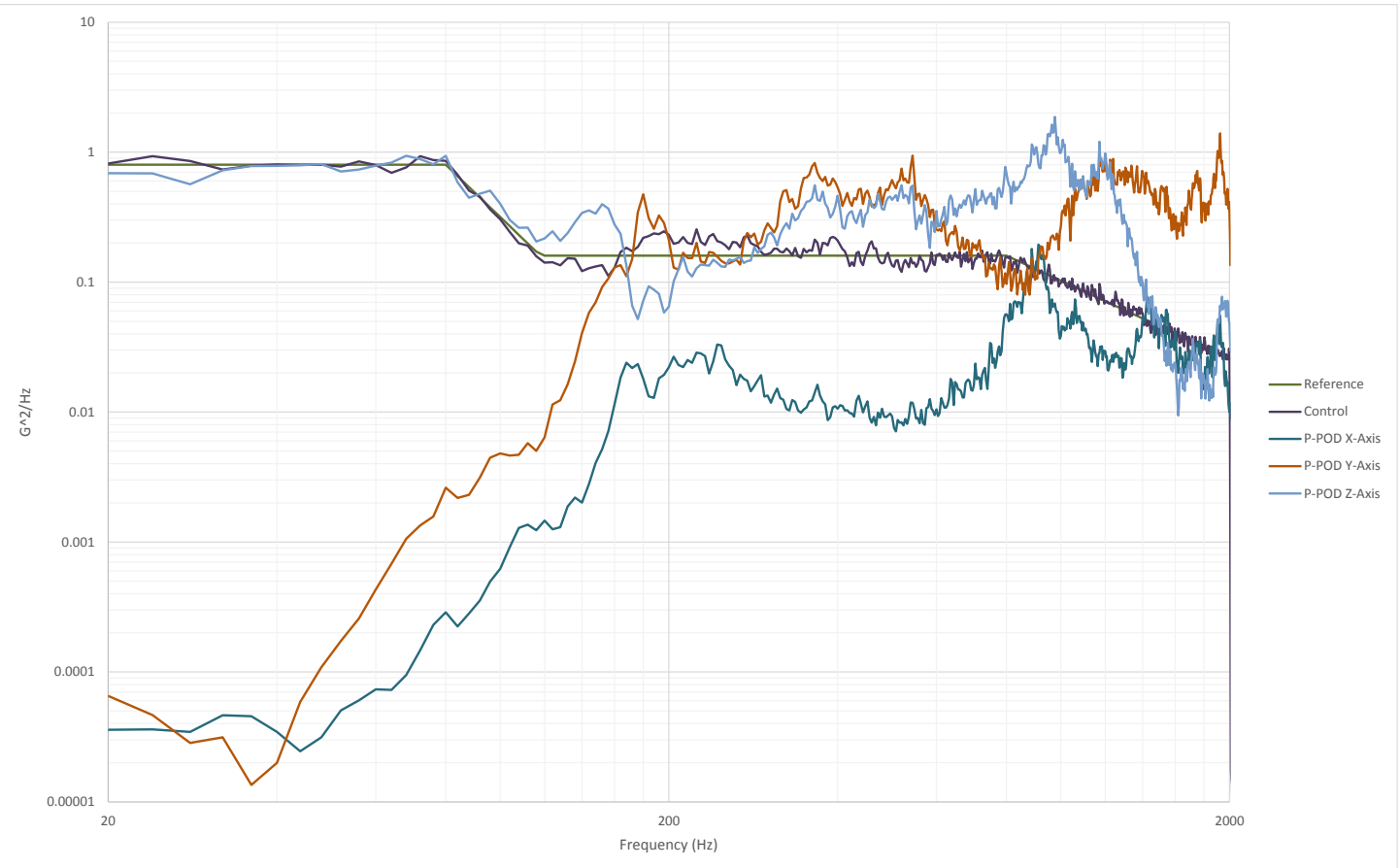

Figure 70 CRS Short Duration Z-Axis P-POD Response Data Page 84 
Post test inspection showed the P-POD was still in functional order following the second random vibration qualification test. At this point a comprehensive inspection is done in accordance with the P-POD Acceptance Checklist (PAC). No exterior damage to the P-POD was observed and all fasteners remained staked and there was no evidence of fasteners backing out. The three fasteners in question after the previous vibration testing exhibited a slight increase in staking delamination, but not complete delamination.

Additionally, torque stripes remained aligned showing that the screws did not back out.

\section{Atlas V Thermal Vacuum Cycling Qualification}

Following completion of all random vibration testing, the P-POD was prepared for thermal vacuum cycling. The thermal vacuum chamber to be used for the thermal vacuum cycling test does not exhibit the best thermal transfer, so for these long duration 8 cycle tests, more direct heating methods are often employed. In this case polyimide film resistive heaters were affixed to the P-POD flat surfaces on the $+/-\mathrm{Y}$ and $+/-\mathrm{Z}$ panels using a low outgassing acrylic adhesive. With these heaters, ramping time is significantly reduced., and the LabView interface is capable of controlling them separately from the standard shroud heaters.

Prior to insertion into the thermal vacuum chamber, the P-POD will have 9 TypeT thermocouples attached to it. The primary areas driving the thermal profile are the PPOD release mechanism and the deployment switches. There are two deployment switches mounted to the $+\mathrm{X}$ side of the bracket, and are wired in parallel so the LV only sees one switch reading. Because of the importance of these two components, two thermocouples were placed on each in case one detached. Another thermocouple was 
placed on the $+Y$ Top Panel near the $-Z$ to read the other end of the P-POD. Additionally, a thermocouple was placed on the $+/-Y$ panels and $+/-Z$ surfaces near the respective heaters on those surfaces in order to monitor heater temperature.

Thermal levels were derived by United Launch Alliance (ULA) and compounded with the specified limits to make up for uncertainty and Qualification standards. The resulting thermal limits at the P-POD Door were $104^{\circ} \mathrm{C}$ and $-28^{\circ} \mathrm{C}$. This is the widest range of temperatures that the P-POD is expected to see from the Launch Vehicles used today. Using these temperatures as dwell levels for eight cycles gives the thermal profile described below in Figure 71.

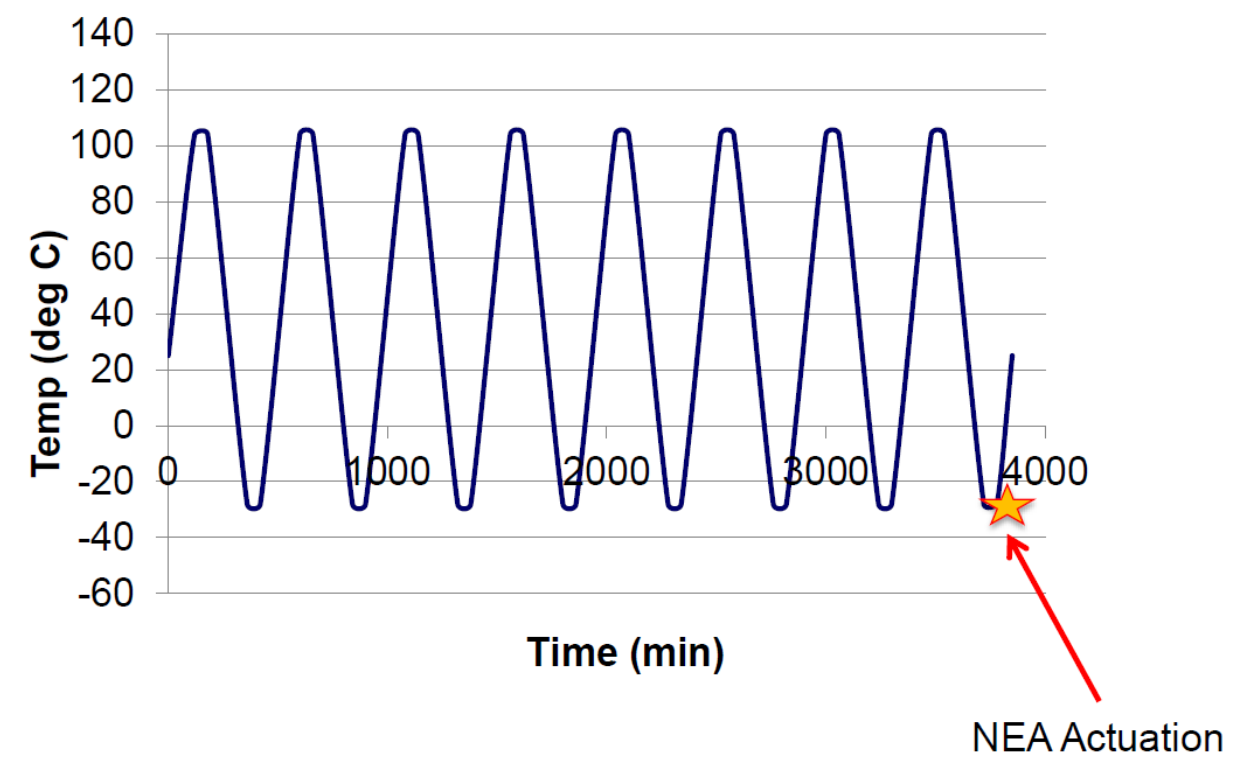

Figure 71 P-POD Qualification Thermal Profile

All NEA and Switch thermocouples must reach the dwell temperature before soaks can be started, and if any of those temperatures fall below/above the specified levels, the soak must be restarted. The NEA Actuation is conducted at the end of the final 
cold soak, while the NEA is still at the cold dwell temperature. In accordance with the program level requirements, the test was conducted at below a pressure of $1.0 \times 10^{\wedge}-4$ Torr. The LabView data acquisition software was used to monitor and record temperatures and control heaters and liquid nitrogen switches that were used to heat and cool the test article in the chamber. Temperature data was automatically recorded by LabView every 30 seconds, and by hand every 10 minutes. Additionally, pressure and resistance measurements were recorded every 10 minutes for the duration of the test.

First Thermal Vacuum Cycling Anomaly

During the first ramp to the hot soak, the P-POD door switch read "OL" which indicates an open door. NEA resistances remainded nominal which was a sign that the door was not inface open, so the P-POD was brought back to room temperature, the chamber re-pressurized, and the P-POD removed and inspected. The switch was indeed open, but instead of being caused by an open door was caused by the door being deformed as a result of the delrin mass model expanding due to the high temperature.

Delrin has a significantly higher coefficient of thermal expansion than Aluminum $\left(5.2 \times 10^{\wedge}-5\right.$ in/in $/{ }^{\circ} \mathrm{F}$ vs. $\left.1.2 \times 10^{\wedge}-5\right)$ which caused it to grow in length significantly more than the Aluminum. After several hours the delrin mass model returned to its initial size. After correspondence with NASA LSP, the decision was made to remove the delrin mass model and integrate $31 \mathrm{U}$ Aluminum mass models that have the same thermal properties as the P-POD panels. 


\section{Second Thermal Vacuum Cycling Anomaly}

Thermal vacuum cycling was restarted, and made it through 2 cycles without issue, but on the third ramp to cold temperature this time, the switch again read "OL". The fast ramp rate (ramped at twice the rate of the first two cycles) suggested that the open reading was caused by the P-POD panels cooling down faster than the mass models, and after reaching thermal equalibrium, the switch closed. The test was continued and the P-POD successfully completed the third cycle and fourth hot soak, but after ramping down at a more modest rate for the $4^{\text {th }}$ cycle, the switch again read open. At this point, the cause of this occurance needed to be determined. After removal from the thermal vacuum chamber, the P-POD was inspected and no obvious issues were apparent. Door to bracket measurements indicated that it is possible that the episode with the delrin mass model caused some permanent deformation to the door in the area of the switch. DoorBracket gap is shown below in Figure 72. This is an uncharacteristic gap in this location, which is right next to the deployment switch that has been causing problem. Further inspection revealed that a 0.010 " gap between the switch guide and switch lever arms were present. Testing on another P-POD with switches mounted in a similar fashion revealed that the point at which the switch opens is at about 0.027 ', suggesting that the door deflected 0.017 " at the switch guide interface. 


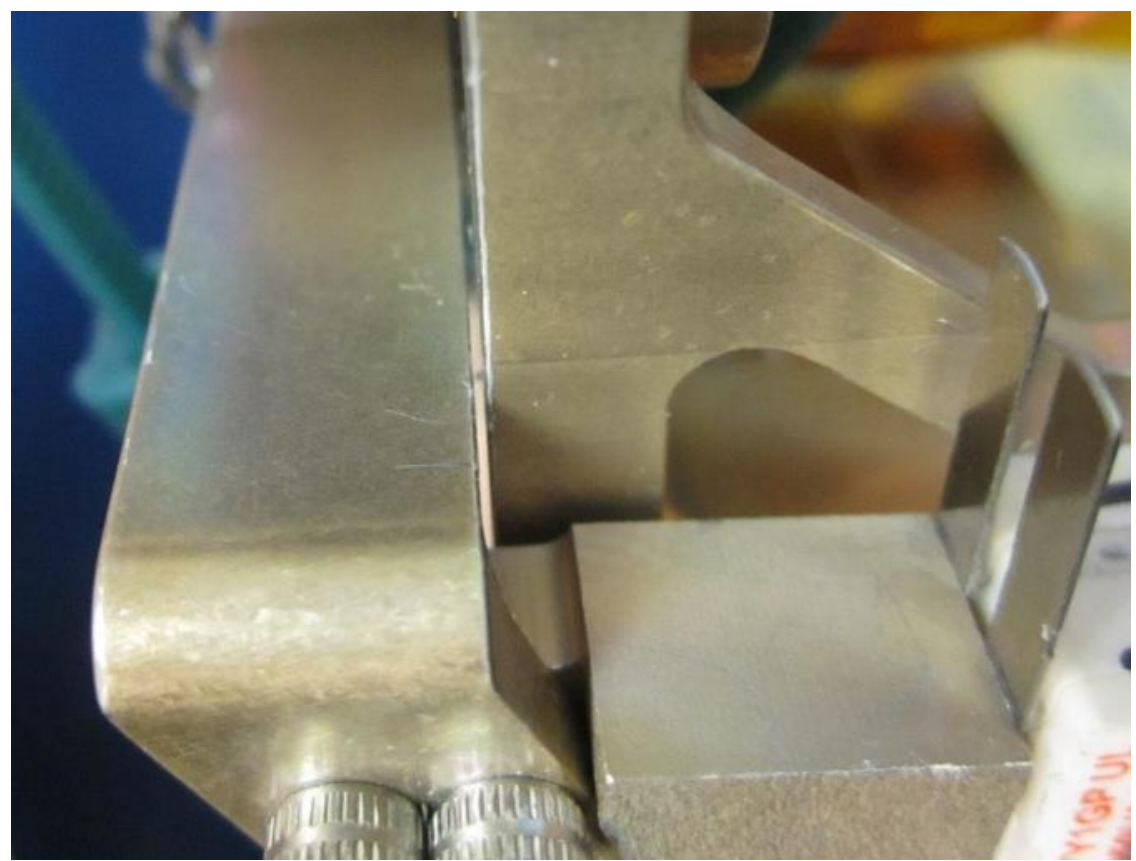

Figure 72 P-POD Door-to-Bracket Gap

\section{Thermal Vacuum Cycling Data}

The temperature levels seen by the NEA and Switch thermocouples during the thermal cycling test are shown below in Figure 73. As shown in the third cold ramp, the ramp was abruptly interrupted when the switch read "OL", and the P-POD temperatures were allowed to warm up slightly and reach equilibrium before the switch circuit had a nominal circuit again. On the fourth cold ramp, the switch opened as soon as the thermocouples reached cold soak temperatures, and eventually closed again after a similar episode of waiting for equilibrium. 


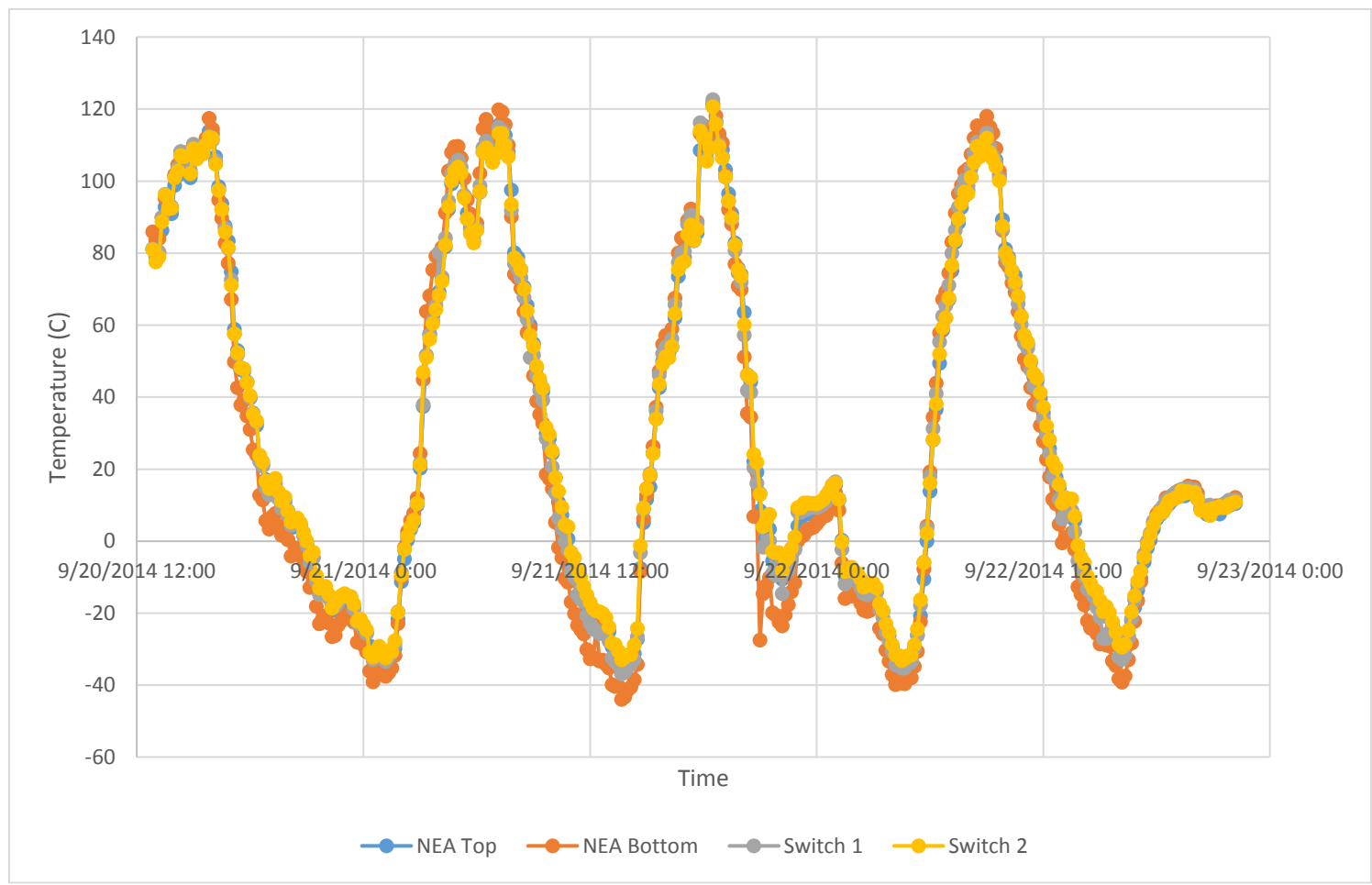

Figure 73 P-POD Control Thermocouple Readings During T-Vac Testing

NEA and Switch resistance reading are shown below in Figure 74 and Figure 75, respectively. There are two NEA resistance measurements because each NEA comes with both a primary and redundant firing circuit, in case of a misfire. Both NEA resistances remained nominal over the entire duration of the test. The two breaks in the switch resistance data curve are the times when the switch was reading "OL". Because it was not possible to complete the thermal vacuum cycling qualification test at this time, the NEA unit on the P-POD was not actuated in T-Vac as is the typical next even in the qualification testing sequence. 


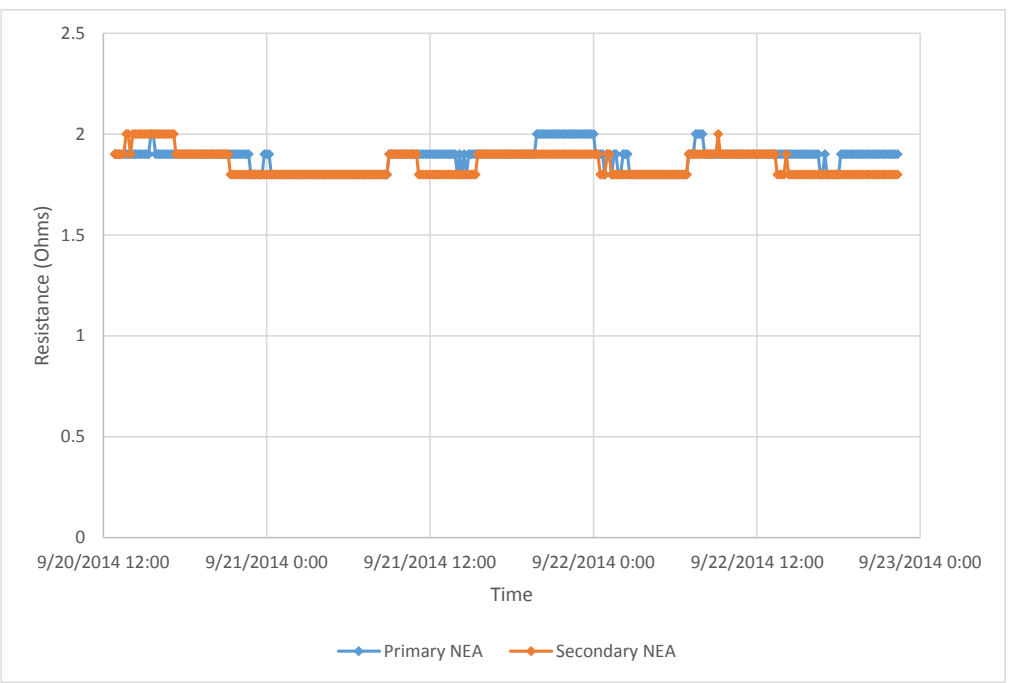

Figure 74 NEA Circuit Resistances During T-Vac Testing

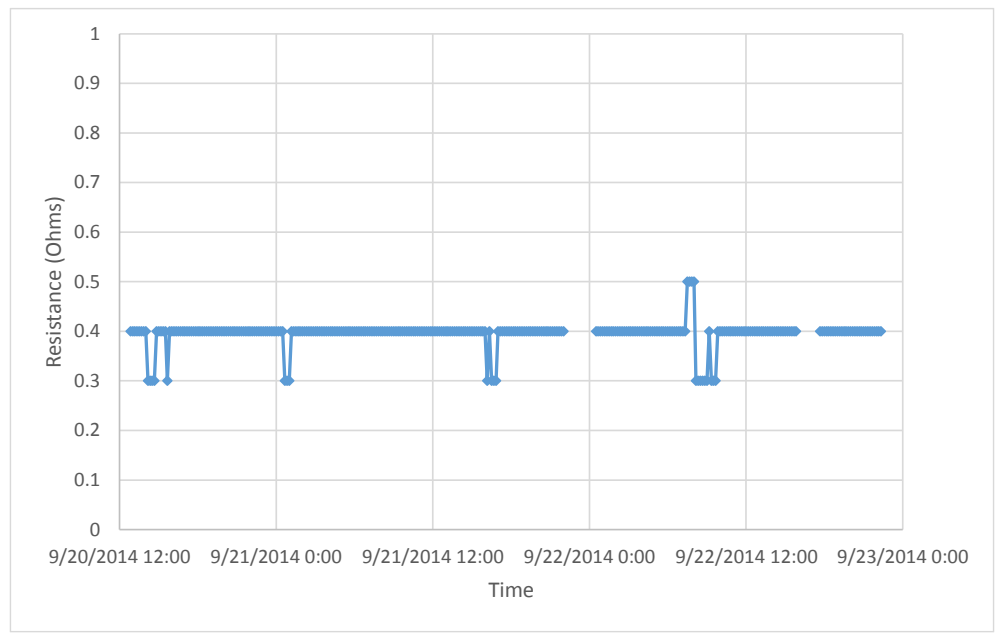

Figure 75 Switch Circuit Resistance During T-Vac Testing

\section{Thermal Vacuum Cycling Fault Tree Analysis}

The leading theory after this anomaly was that the mass models had caused the door to deflect enough to open the switch. This corresponds to a relatively large 
temperature differential between the Aluminum mass models and the P-POD exterior. The thermal expansion of aluminum being described as follows,

$$
d l=L_{0} \alpha \Delta t
$$

Equation 8

Where $L_{0}$ represents the initial length, $d l$ is the change in length from thermal expansion, $\alpha$ is the coefficient of linear thermal expansion, and $\Delta t$ is the temperature differential. In this case, the length of the Aluminum Mass Models and the Pusher Plate is 14.84". To simplify the problem, the exterior panel length was assumed to be the same length, and the change in length was set to the theoretical door deflection of 0.017”, with the intent being to solve the equation for the change in temperature that would cause the deflection. After solving the equation and converting to metric units, the temperature differential that would cause a deflection of 0.017 ' is $48.58^{\circ} \mathrm{C}$. This is a relatively large gradient, but is not unheard of. Additionally, given that the temperature gradient across the P-POD from $+Z$ Door to $-\mathrm{Z}$ Back Plate reached $30^{\circ} \mathrm{C}$ at the time of the anomaly, it is reasonable to assume that the aluminum mass models were lagging further behind.

At this point, it was necessary to conduct a top down fault tree analysis so ensure that the actual cause of this anomaly is determined, so it can be prevented from happening again. The list of faults investigated is show below in Table 16. The first three entries in the table above have already been discussed, and there is evidence that suggests that all three are related and contributed to the anomaly that occurred during T-Vac. The initial gap between the switch and switch guide, caused by slight door deformation from the delrin mass model incident lowered the required temperature differential between the P-POD exterior and Aluminum mass models required to deflect the door enough to cause 
a false open reading on the switch. An insufficient pre-load on the release bolt that holds the door closed was considered, but the torque wrench used was tested using a torque tester and measured well within tolerance for the 50 in-lb torque setting. Additionally, the presence of the door-collar gasket was investigated. The collar gasket material has a low thermal conductivity $\left(2.2 \mathrm{~W} /\left(\mathrm{m}^{*} \mathrm{~K}\right)\right.$ vs $\left.237 \mathrm{~W} /\left(\mathrm{m}^{*} \mathrm{~K}\right)\right)$, so the concern was that the collar gasket would not cool down at the same rate of the P-POD and would create a larger gap in the door causing the switch to read open. The gasket however is hollow, and is 0.083 " wide before it is compressed, so the change in width resulting from a temperature change would be insignificant.

Table 16 Fault-Tree Analysis Investigation

\begin{tabular}{|c|c|c|}
\hline High Level Cause & Specific Cause & Probability \\
\hline \multirow{5}{*}{ Door Gapping } & Initial Switch Gap & High \\
\hline & $\begin{array}{c}\text { P-POD to Mass Model Temperature } \\
\text { Differential }\end{array}$ & High \\
\hline & Door Deformation & High \\
\hline & Insufficient NEA Preload & Low \\
\hline & Collar Gasket Thermal Expansion & Low \\
\hline \multirow{3}{*}{$\begin{array}{l}\text { Bad Switch/ Switch } \\
\text { Guide Configuration }\end{array}$} & Mk. IV Switch Guide Geometry & Medium \\
\hline & Thermal Cycling Shifting Switch Position & Low \\
\hline & Switch Malfunction & Low \\
\hline \multirow{3}{*}{ Faulty Connection } & Bad Solder Joint on Switch Harness & Low \\
\hline & Connector Malfunction & Low \\
\hline & T-Vac Chamber Faulty Wiring & Low \\
\hline
\end{tabular}

The next high level cause of this occuring, is a problem with the switch/switch guide configuration. At this point, the design of the switch guide came under scrutiny. With a much thinner profile than the previous design, the guide does not have as much 
contact with the deployment switch levers as before. The Mk. IV Switch Guide is shown below in Figure 76. Having more contact with the lever can keep the switch depressed

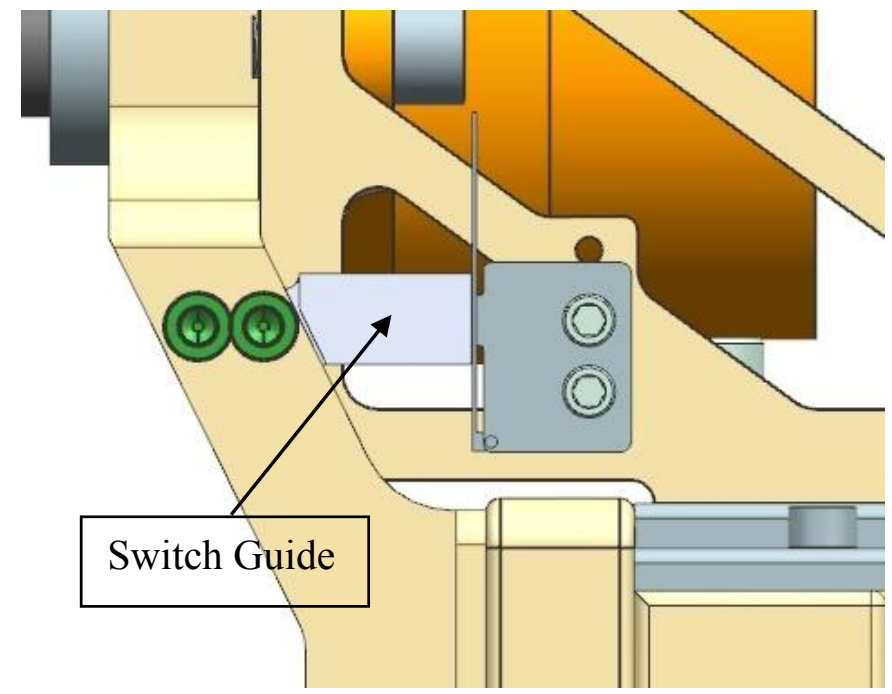

Figure 76 P-POD Mk. IV Switch Guide Design

even with some gapping because of the potential geometry and angular incidence of the switch guide on the switch. This was identified as a moderately probable contributor to the anomaly that occurred. In order to rectify this issue, a redesigned switch guide was proposed. The goal of the design was to provide a switch to switch guide interface that would be more tolerant of door gapping to prevent any false open readings. In addition to having a greater contact surface on the switch levers, having the switch guide push against the levers such that they deflect past the face of the switch allows the switch to stay engaged even if the door starts to gap. Additionally, it is slightly longer, designed to nominally account for common door gaps. The modified switch guide design is shown below in Figure 77. 


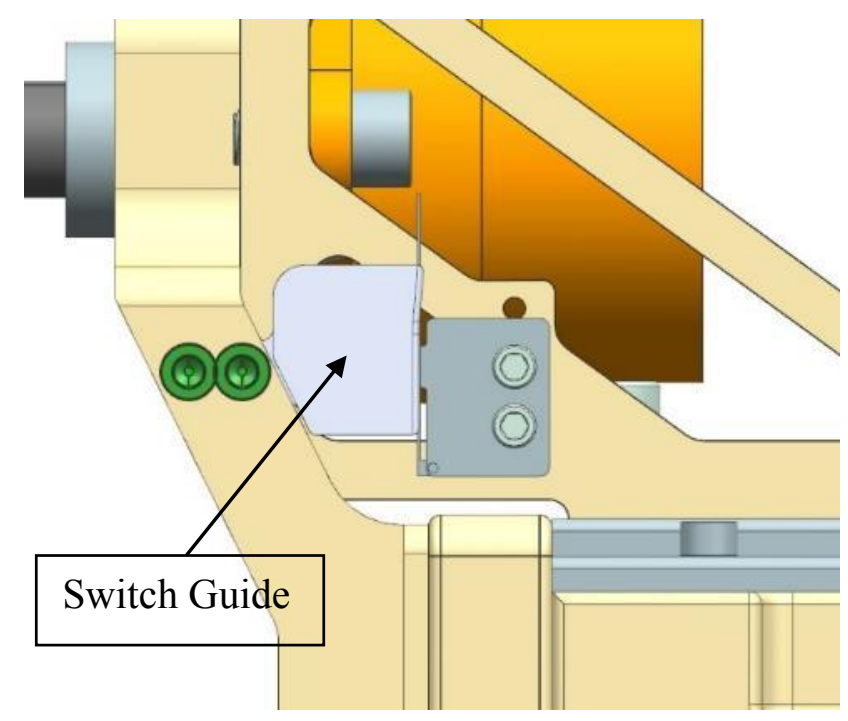

Figure 77 Modified Mk. IV Switch Guide Design

Other possible issues with the switch and/or switch guide configuration include movement of the switch at some point during thermal cycling or the switch itself malfunctioning. The switch shifting is unlikely because such an occurance would cause the staking on the switch screws to delaminate from either the switch or the switch screws. There is no visible evidence of either occuring, and putting a moment on the harness shows no movement of the switch. The switch actually malfunctioning as a result of the temperature extremes is also unlikely for a few reasons. The anomaly occurred while the chamber was ramping cold, and the first time it occurred, it was not even close to the extreme. Additionally, the switch is rated to $-40{ }^{\circ} \mathrm{C}$, and the lowest the switch thermocouples read were $-32.6^{\circ} \mathrm{C}$. Finally, the same model switch has been qualified in T-Vac to these same levels before, so it is unlikely that the anomaly would be associated with the temperature extreme causing damage to the switch. 
Another high-level fault that would cause this sort of an anomaly would be a bad connection at some point in the P-POD harness, T-Vac chamber wiring, or the connector itself was malfunctioning due to the thermal levels. If there was a problem in the P-POD harness, it would be caused by a solder joint on the switch losing continuity. The two switches on the P-POD are connected in parallel so in the event of one switch losing continuity as a result of a bad solder joint, the other switch would continue to read nominal and there would be no "OL" reading on the switch. The T-Vac wiring issue was ruled out because there has never been an issue with the T-Vac wiring prior to this occurance, and the likelihood of it occuring on only the switch circuit is suspect, as evidence suggests that it is possible that door gaps were increasing during the test. Also, it would be odd to have a wiring issue when ramping down to cold temperatures but not at hot temperatures. Lastly, the Amphenol MIL-DTL-38999 connector is rated from $+200{ }^{\circ} \mathrm{C}$ to $-65{ }^{\circ} \mathrm{C}$, so it is very unlikely that a connector with this operating range would fail near $-30{ }^{\circ} \mathrm{C}$.

\section{P-POD Mk. IV Thermal Vacuum Testing Path Forward}

After examining the possible causes and likelihood of each, it was determined that the anomaly was most likely caused by the temperature differential between the P-POD exterior panels and the integrated aluminum mass models, which was exacerbated by the intial gap between the switch and switch guide, along with the Mk. IV switch guide design that poorly utilizes the switch lever arm to maintain engagement, which both combined to reduce the temperature differential required to cause the door to gap and open the switch. This is the first time in the history of the CubeSat program that this has occurred, and has been a very good example of why thermal vacuum cycling is done for 
qualifying a design. In the future, greater care will be taken in reviewing the coefficients of thermal expansion for all materials, and ensuring that the switch to switch guide interface is nominal, and all future switch guide designs take full advantage of the lever arms on the switch to prevent this anomaly from occuring again.

\section{P-POD EMI Attenuation Testing}

The next step in this testing sequence was to prove the P-POD could provide adequate EMI shielding. The desired amount of shielding was $50 \mathrm{~dB}$ for frequencies from $400 \mathrm{MHz}$ to $10 \mathrm{GHz}$. Instead of testing the entire frequency range, special attention was given to commonly used frequencies such as $437 \mathrm{MHz}$ and $900 \mathrm{MHz}$ (UHF), 2.4 $\mathrm{GHz}$ and 5.6 GHz (Wi-Fi and S-Band), and $10 \mathrm{GHz}$. Ultimately, only a selection of these frequncies were tested for reasons discussed below. Testing was done adhering to the IEEE Standard Method for Measuring the Shielding Effectiveness of Enclosures and Boxes Having all Dimensions between $0.1 \mathrm{~m}$ and $2 \mathrm{~m}$ (IEEE Std 299.1 ${ }^{\mathrm{TM}}-2013$ ) wherever possible. According to the IEEE Std 299.1-2013, RF testing should only be done for frequencies greater than 3 times the resonant frequency, or in this case, $4.3 \mathrm{GHz}$. This presents a problem in that most of the frequenceis we are most interested in reside below this value. This makes it likely that the data measured from this test was not accurate, and can help explain the results to some degree. A preliminary test was conducted in order to become familiar with the testing equipment and make sure all necessary equipment was available. The test was conducted in Cal Poly's Anechoic chamber using with an HP $8720 \mathrm{C}$ Vector Network Analyzer and a selection of different types of antennas. Monopole antennas tuned for the specific frequencies of interest were mounted inside the P-POD in order to receive the signal from a higher gain transmitting 
antenna, and compared to an identical system, but without the P-POD, to determine the change in signal strength, in $\mathrm{dB}$. This change is described as attenuation, as it is the PPOD enclosure partially isolating the receiving antenna inside the P-POD from the transmitting antenna roughly 2 meters away.

\section{Dynamic Range Testing, $437 \mathrm{MHz}$}

Before attenuation testing began, it was necessary to first determine the dynamic range of the testing system. This is determined by first taking the measurement with both the transmitting and receiving antennas in the chamber, with no obstructions. Then, unplugging one of the antennas and taking the same measurement. This is the equivalent of completely isolating the antennas from one another. The difference of these two measurements is called the Dynamic Range. It is important because if the dynamic range is less than $50 \mathrm{~dB}$, then $50 \mathrm{~dB}$ of attenuation can never be achieved. A perfectly enclosed box that would theoretically contain any RF transmissions, would show an attenuation level equal to the Dynamic Range.

Initially, the hope was to use one setup for the entire range of testing. Two monopole antennas tuned to $2.4 \mathrm{GHz}$ were going to be used for the entire frequency range, but unfortunately, upon determining the Dynamic Range it was clear that this setup would not be sufficient. Instead, a frequency matched monopole was used inside of the P-POD, and a higher gain transmitting antenna was used for each frequency. For the 437 MHz range, a Sinclair Technologies Model No. SY307-SF2SNM directional Yagi- Uda antenna was used as the transmitter, providing a $10 \mathrm{~dB}$ increase in gain. This antenna is shown below in Figure 78. 


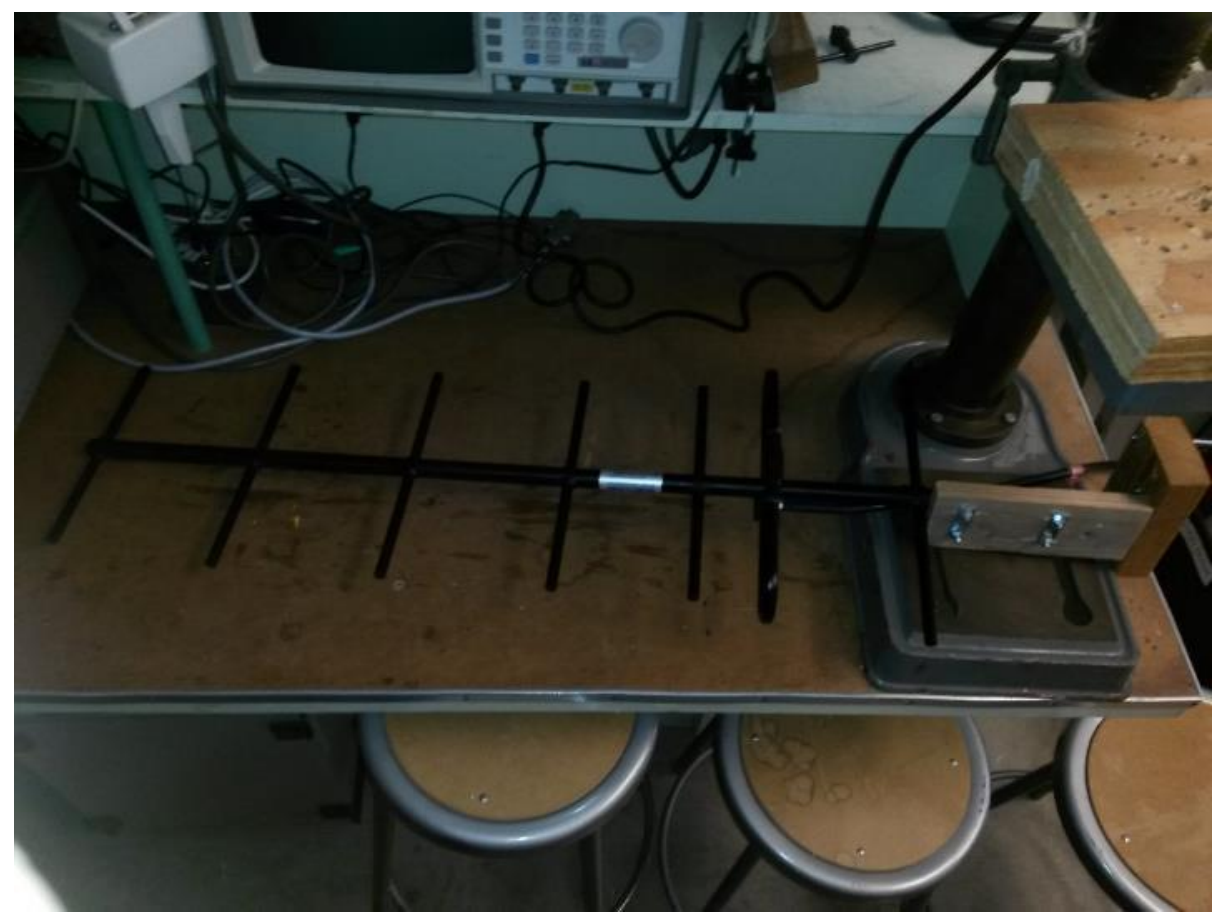

Figure $78 \quad 437 \mathrm{MHz}$ Sinclair Yagi-Uda Antenna

Unfortunately, this was still insufficient, and an inline amplifier was added to the receive signal. This brought the Dynamic Range to the $59 \mathrm{~dB}$ level, which is close to being enough, but still insufficent. The resulting dynamic range for this setup over a range of frequencies is shown below in Figure 79. 


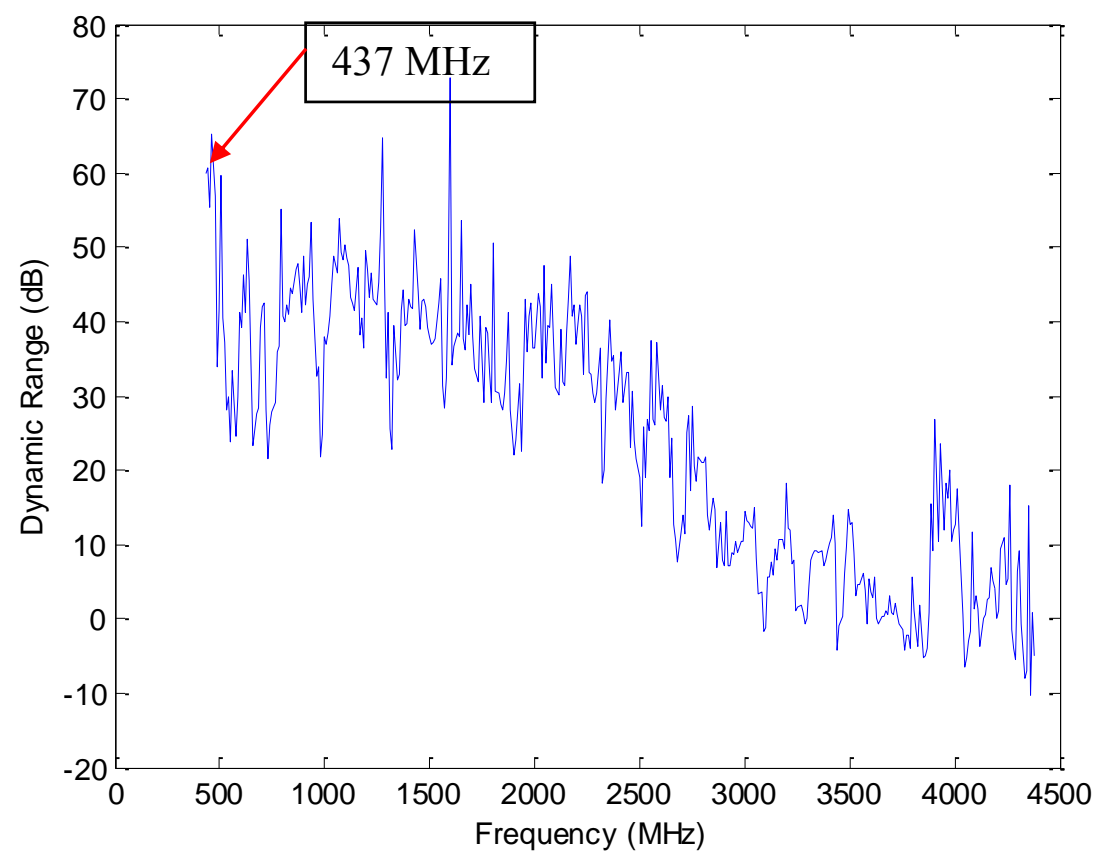

Figure 79 Anechoic Chamber Dynamic Range

As the shielding measurement gets close to the Dynamic Range its linearity begins to break down. It was advised by an RF specialist at NASA KSC that $70 \mathrm{~dB}$ of Dynamic Range was needed in order to measure a shielding effectiveness of $50 \mathrm{~dB}$. The hardware available at the time of the test was not going to make this possible, so the test was conducted for the $437 \mathrm{MHz}$ range and the $5.6 \mathrm{GHz}$ range, to ensure the P-POD was attenuating an appreciable amount given the deficit in dynamic range.

\section{MHz P-POD Attenuation Testing}

The measurement between the pair of antennas was compared to measurements with the receiving antenna located in the P-POD, in different orientations. These orientations included through the + Y Top Panel, at a $45^{\circ}$ angle splitting the Top Panel 
and $+\mathrm{X}$ Side Panel, $+\mathrm{X}$ Side Panel, and a $45^{\circ}$ angle splitting the $-\mathrm{Y}$ Bottom Panel and the $+X$ Side Panel.

\section{$437 \mathrm{MHz}$ P-POD Attenuation Testing Results}

The RF signal strength between the transmit and receive antennas over a narrow range of frequencies at each orientation, along with the noise floor (minimum measurement), is shown below in Figure 80. In all three orientations, the P-POD

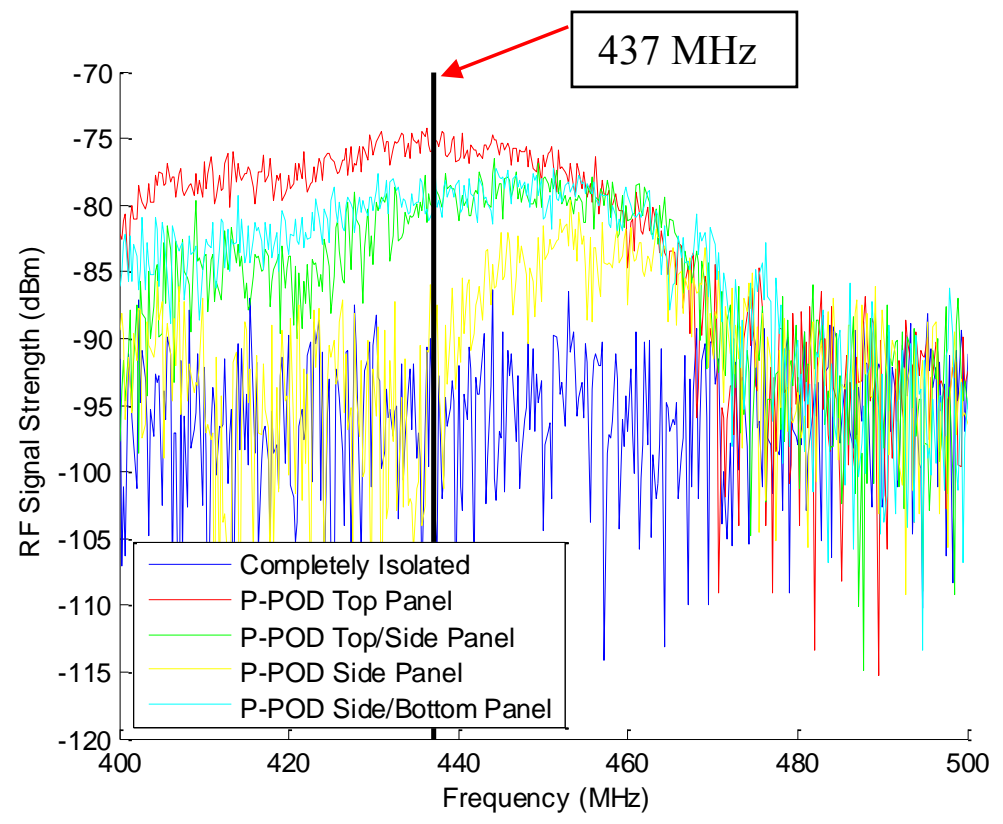

Figure $80 \quad$ RF Signal Strength Measurements at 400-500 MHz

attenuates a significant amount, but without the necessary dynamic range the desired attenuation cannot be measured. Given the dynamic range, when the measured signal strength with the P-POD was compared to the signal strength measured between the two antennas, the P-POD attenuates the following values shown below in Table 17. It is clear that the Top Panel, with its array of small venting holes, is attenuating worse than the 
Side Panel. The first and most obvious explanation of this would be that the venting holes that were sized and spaced to shield the frequency range in question are not attenuating EMI as they should according to the analysis.

Table 17 P-POD Shielding Effectiveness at $437 \mathrm{MHz}$

\begin{tabular}{|c|c|}
\hline Orientation & Shielding Attenuation $(\mathrm{dB})$ \\
\hline Top Panel $(+\mathrm{Y})$ & 38.34 \\
Top Panel/Side Panel $(+\mathrm{Y},+\mathrm{X})$ & 42.88 \\
Side Panel $(+\mathrm{X})$ & 53.11 \\
Side Panel/Bottom Panel $(+\mathrm{X},-\mathrm{Y})$ & 42.81 \\
\hline
\end{tabular}

According to the EMPro analysis, at this frequency the Top Panel should be capable of providing $81 \mathrm{~dB}$ of attenuation, so other possible causes were investigated. The attenuation of the P-POD at a $45^{\circ}$ angle was greater than that of the Top Panel which would be expected given that the venting holes are no longer normal to the RF source. However, the attenuation of the P-POD at the $45^{\circ}$ angle splitting the Side Panel and Bottom Panel was the same, which does not suggest that the venting holes play a part in the loss of attenuation, as there are no venting holes, or any other holes, on the P-POD Bottom Panel. The Side Panel data is on top of the noise floor, so the actual value is likely not accurate, but it safely produces at least $50 \mathrm{~dB}$ of attenuation according to the data. Another cause of the difference between the panels could be that the receive antenna translates slightly as the P-POD is rotated, causing a much weaker signal. This could have been rectified by actually measuring the open antenna signal strength at the correct orientation, but the test operators constraints on time limited this ability for this test. In any case, the original theory stands that the dynamic range of $59 \mathrm{dBm}$ is not 
sufficient to measure $50 \mathrm{~dB}$ of attenuation, because as attenuation gets close to dynamic range it no longer accurately represents the actual attenuation offered by the specimen and reads under what its actual value is.

Additionally, one clause in the IEEE Std. used to conduct this test pertained to RF resonance and the resonant frequency of the test specimen. According to the IEEE Std 299.1-2013, the lowest possible resonant frequency for a rectangular box with the dimensions of the P-POD is given by

$$
f_{r}=\frac{1}{2 \sqrt{\mu \varepsilon}} \sqrt{\left(\left(\frac{1}{a}\right)^{2}+\left(\frac{1}{b}\right)^{2}\right)}
$$

Where $\mu$ is the permeability of free space inside the enclosure, $\varepsilon$ is the permittivity of free space, $a$ is the longest dimension of the P-POD, and $b$ is the intermediate dimension of the enclosure. Both length dimensions are in meters. Plugging in the appropriate values, this equation becomes

$$
f_{r}=150 \sqrt{\left(\left(\frac{1}{0.381}\right)^{2}+\left(\frac{1}{0.110}\right)^{2}\right)}=1419.3 M H z \quad \text { Equation } 10
$$

\section{Dynamic Range Testing, $5.6 \mathrm{GHz}$}

In an effort to try to test another frequency above the $4.3 \mathrm{GHz}$ limit, the next critical frequency was $5.6 \mathrm{GHz}$. The correct antennas for this frequncy range were available, but unfortunately no amplifier was available to boost the dynamic range. The test was resumed anyway to confirm that the P-POD was at least measuring the maximum attenuation it could given the low dynamic range. 
When testing the $5.6 \mathrm{GHz}$ range, the dynamic range was even less, because the only amplifier available was for a frequency range of 400-500 MHz, and therefore the amplifier did little to boost the dynamic range, which was measured to be much less than it was before. For this setup, again a monopole antenna tuned for the $5.6 \mathrm{GHz}$ frequency was used as the receiving antenna on the interior of the P-POD. The exterior transmitting antenna was a Narda standard gain horn antenna, Model 642, tuned for the correct frequency and is shown below in Figure 81. The dynamic range of the setup in this frequency range was measured to be just $25.75 \mathrm{~dB}$, which is not nearly sufficient to measure the levels of attenuation that are desire. A plot of dynamic range from frequencies of $5.5 \mathrm{GHz}$ to $5.7 \mathrm{GHz}$ is shown below in Figure 82 .

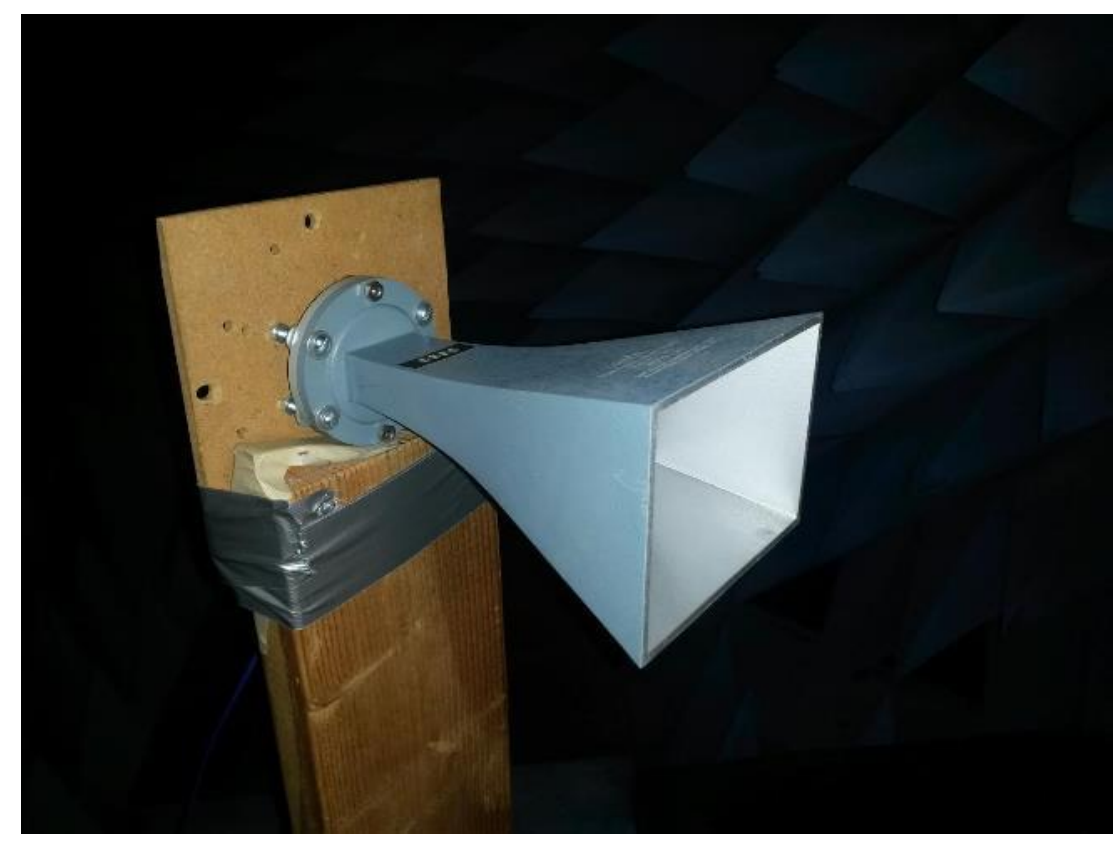

Figure 81 Horn Antenna Used for 5.6 GHz EMI Testing 


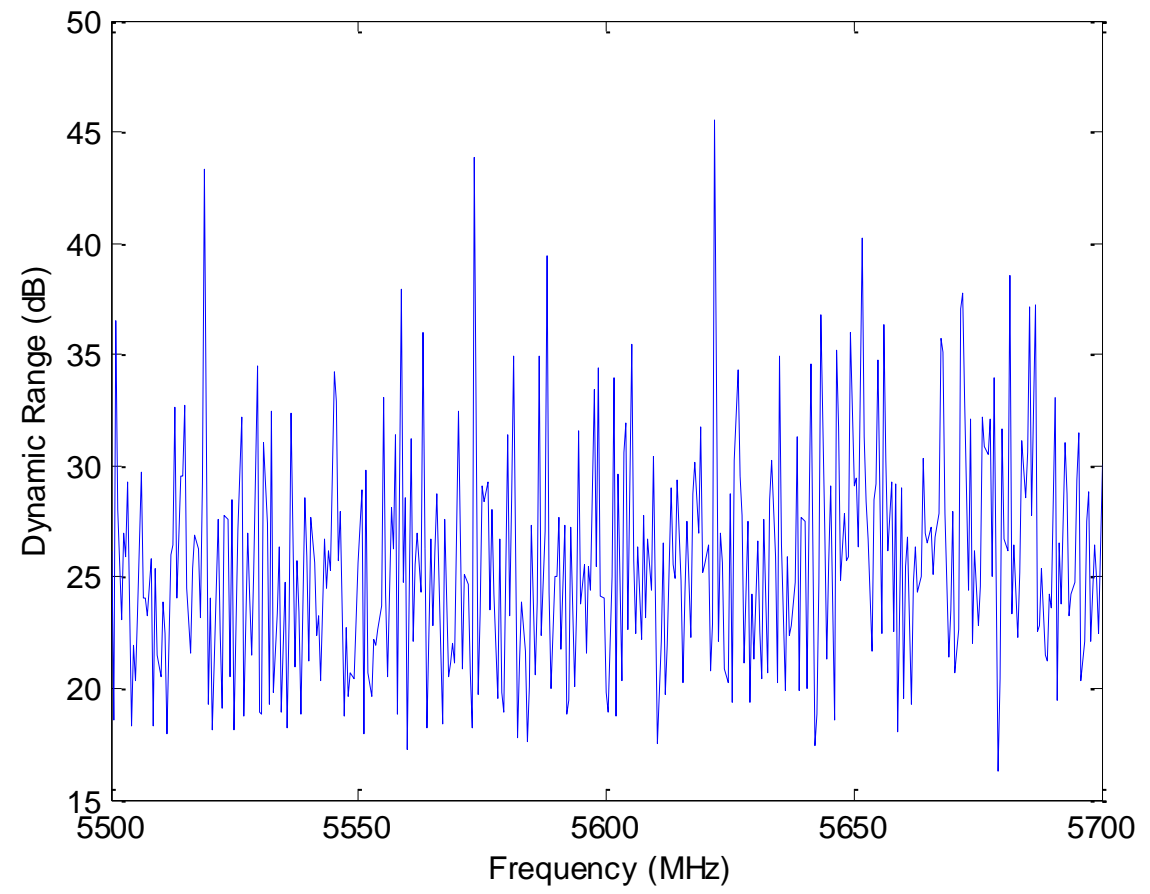

Figure 82 Dynamic Range of Anechoic Chamber for 5.6GHz Range

\subsection{GHz P-POD Attenuation Testing}

It was clear that some changes in setup would need to occur, including using some sort of amplification on the transmitting side. Unfortunately, with the equipment available the test was conducted anyway to verify that the P-POD would maintain its RF shielding at this frequeny range. For the $5.6 \mathrm{GHz}$ range, only the first 3 orientations were measured, excluding the measurement that was split between the Side Panel and Bottom Panel.

\subsection{GHz P-POD Attenuation Testing Results}

As expected, the measured signal strength with the receive antenna surrounded by the P-POD was right on top of the noise floor, showing that the P-POD attenuates much 
more than the $25.75 \mathrm{~dB}$ dynamic range. The measured signal strengths at each orientation is shown, along with the noise floor, below in Figure 83. Because of the noise, it is difficult to ascertain an exact value, but it is pointless as it is clear the P-POD attenuates more than the $25.75 \mathrm{~dB}$ dynamic range in this frequency range.

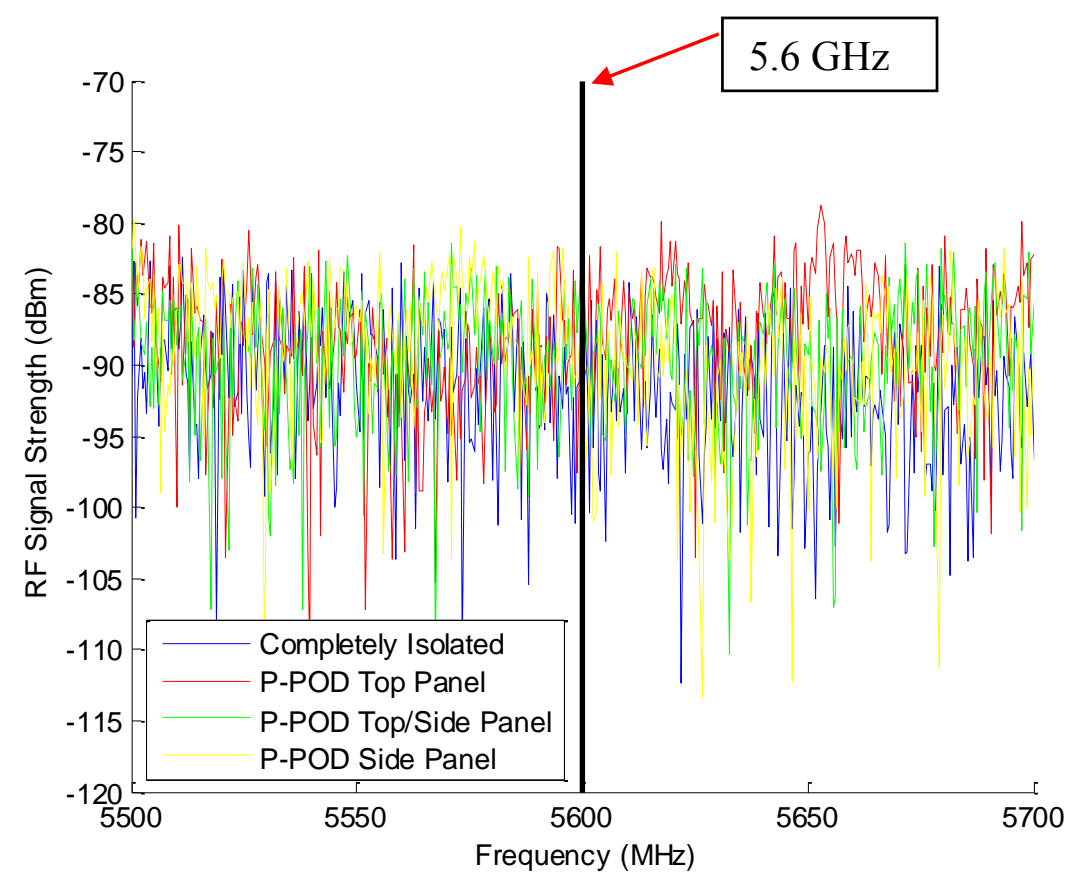

Figure 83 P-POD Attenuation Measurement at $5.5 \mathrm{GHz}$ to $5.7 \mathrm{GHz}$

\section{P-POD Attenuation Testing Summary and Path Forward}

Two different cases were tested, the first was determined to be a questionable test based on the fact that the frequency tested was below the resonant frequency of the PPOD. Additionally, during the first test, there was not quite enough dynamic range available in the setup in order to reach. This issue was worse in the second test at 5.6 $\mathrm{GHz}$, because no amplifiers were available at that frequency range, and further more, there are more line losses at higher frequencies. Moving forward, to test EMI again to 
adequate levels, a set of amplifiers would need to be purchased in order to reach the required dynamic range. These amplifiers are very expensive, some cost over $\$ 2000$ to meet the required specifications. It could also be more effective to move to test the system at a dedicated $3^{\text {rd }}$ party testing facility to ensure that testing results are as accurate as they can be and all levels are met. 


\section{CHAPTER VI: CONCLUSION AND FUTURE WORK}

The Poly-Picosatellite Orbital Deployer has undergone a series of improvements over the years. This Master's Thesis has built on these improvments, taking them further and offering new capabilities and features that were not available before. The structural design of the P-POD was modified to reduce mass that was unnecessary for the structural survivability, while making the weak point of the P-POD at the door hinge stronger. This mass reduction and strengthening has allowed more mass to be allotted to the CubeSat paylaod while being capable of handling the increase in load from the increased payload mass. Additionally, a design has been put into place that will allow CubeSat payloads access to power and data during launch and opening the door to countless mission opportunities that previously did not exist. Alongside the Power-On system, EMI shielding has been incorporated into the P-POD to allow the CubeSats to turn on within the P-POD without negatively effecting the launch vehicle or the primary spacecraft. The EMI shielding design has been anaylzed and shown to be effective, and testing results show promise but available testing equipment limited the success of the testing sequence. In addition to EMI Shielding and CubeSat access to power and data, an inert gas purge system has been designed, tested, and flown on a P-POD Mk. III, and proved to be an effective design. The appropriate sections of the P-POD have been designed to accommodate the addition of both the inert gas purge system, the Power-On electrical system, or a combination of the two, whenever a specific mission requires it. The P-POD Mk. IV was successfully qualified to the random vibration environment on both the NPSCuL, specified by the ULA Atlas V Aft Bulkhead Carrier User Guide, and the environment specified by SpaceX for flight on the Falcon $9 \mathrm{LV}$, and passed both without 
any structural yielding or failure. The P-POD Mk. IV also underwent qualification thermal cycling to the levels determined by ULA, but was cut short due to an anomaly experienced during T-Vac. Future work can focus on both the EMI testing and getting the P-POD through thermal vacuum cycling.

The thermal vacuum cycling issue is in part a result of the first anomaly with the Delrin mass model that caused a slight permanent deformation in the door that left a gap between the switch and switch guide. The $2^{\text {nd }}$ anomaly occurred most likely as a result of this gap that was not present before, combined with a switch guide design that did not fully take advantage of the switch lever arm that helps to keep the switch engaged. In order to rectify this issue, a new design of the switch guide, outlined in this thesis, can be implemented to maintain switch engagement during non-catastrophic deformation occurences. Ultimately, the anomaly most likely would not have occurred had the door not been slightly deformed producing the switch to switch guide gap, but this identified a sensitive area of the P-POD, and implementing the new design would serve to strengthen this area of the P-POD for the next test and the future.

The EMI testing is a slightly more complex issue. The lack of attenuation measured during the EMI testing stems from a significant amount of signal losses in the anechoic chamber testing equipment, which manifest themselves in a severely lacking dynamic range. This issue was especially apparent in the higher frequency testing because no amplifier was available in the correct frequency range. In order to raise the dynamic range, more high-gain, low noise amplifiers need to be purchased in order to boost the transmitting signal to yield more of an opportunity to measure attenuation. 
Multiple amplifiers are needed because each one is designed for a specific frequency range. The other option is to utilize a professional testing facility that will have all of the necessary equipment conduct the test. In the future, after itemizing the appropriate amplifiers to get to the desired dynamic range, the cost and likelihood of success after incorporating those amplifiers needs to be assessed and compared to getting the test done at a professional facility, and then go about acquiring the funds to do one or the other.

Additionally, because part of the testing is conducted under the recommended minimum frequency for testing ( 3 times the resonant frequency), the results of the testing under that frequency will be subject to scrutiny and simply used as reference data wherever possible.

The P-POD Mk.IV, shown below in Figure 84, has opened up new avenues for CubeSat exploration offering new features for the advancement of space technology and education. There are still several aspects that can be improved upon in the future.

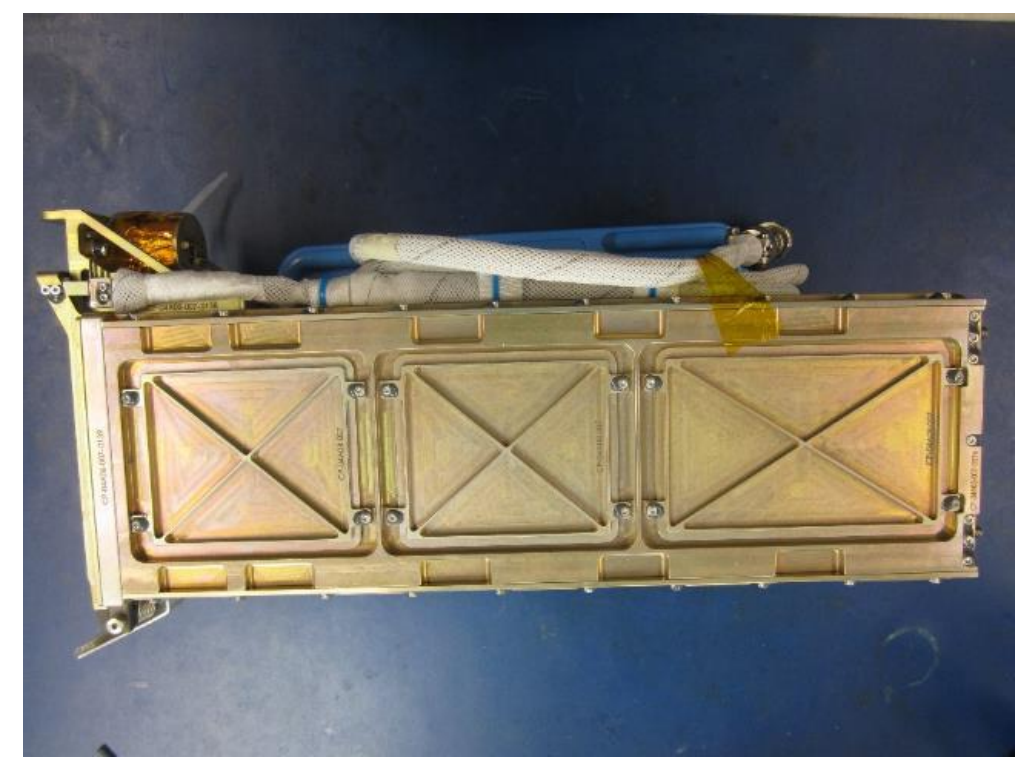

Figure $84 \quad$ P-POD Mk. IV Engineering Unit Page 110 
The P-POD Mk. IV Door could see improvements from a more aggressive redesign, removing the stress concentration from the door hinge. Environment mitigation is also of interest. Making the CubeSat's ride into space less harsh is a top priority in the industry. Some concepts exist for vibration isolation and thermal shielding, and incorporating those concepts into a CubeSat deployer is the next big step for the P-POD design. 


\section{REFERENCES}

Irvine, (2005), The Half Power Bandwidth Method for Damping Calculation [ONLINE].

Available at: http://www.vibrationdata.com/tutorials2/half_power_bandwidth.pdf

Irvine, (2009), An Introduction to the Vibration Response Spectrum [ONLINE].

Available at: http://www.vibrationdata.com/tutorials2/vrs.pdf

Military Specification (MIL)-STD-1540C, Test Requirements for Launch, Upper-Stage, and Space Vehicles, revision C (15 September 1994).

IEEE Standard Method for Measuring Shielding Effectiveness of Enclosures and Boxes

Having all Dimensions between 0.1 m and 2 m, IEEE Standard 299.1, 2014. 